University of Rhode Island

DigitalCommons@URI

Open Access Master's Theses

1995

\title{
Computer Simulation of Wave Propagation and Dynamic Load Transfer Through Saturated Particulate Media
}

Gautam Adhikari

University of Rhode Island

Follow this and additional works at: https://digitalcommons.uri.edu/theses

\section{Recommended Citation}

Adhikari, Gautam, "Computer Simulation of Wave Propagation and Dynamic Load Transfer Through Saturated Particulate Media" (1995). Open Access Master's Theses. Paper 875.

https://digitalcommons.uri.edu/theses/875

This Thesis is brought to you for free and open access by DigitalCommons@URI. It has been accepted for inclusion in Open Access Master's Theses by an authorized administrator of DigitalCommons@URI. For more information, please contact digitalcommons-group@uri.edu. 
COMPUTER SIMULATION OF WAVE PROPAGATION AND DYNAMIC LOAD TRANSFER THROUGH SATURATED PARTICULATE MEDIA BY GAUTAM ADHIKARI

A THESIS SUBMITTED IN PARTIAL FULFILLMENT OF THE REQUIREMENTS FOR THE DEGREE OF MASTER OF SCIENCE IN MECHANICAL ENGINEERING AND APPLIED MECHANICS 


\section{MASTER OF SCIENCE THESIS}

OF

\section{GAUTAM ADHIKARI}

\section{APPROVED :}

Thesis Committee

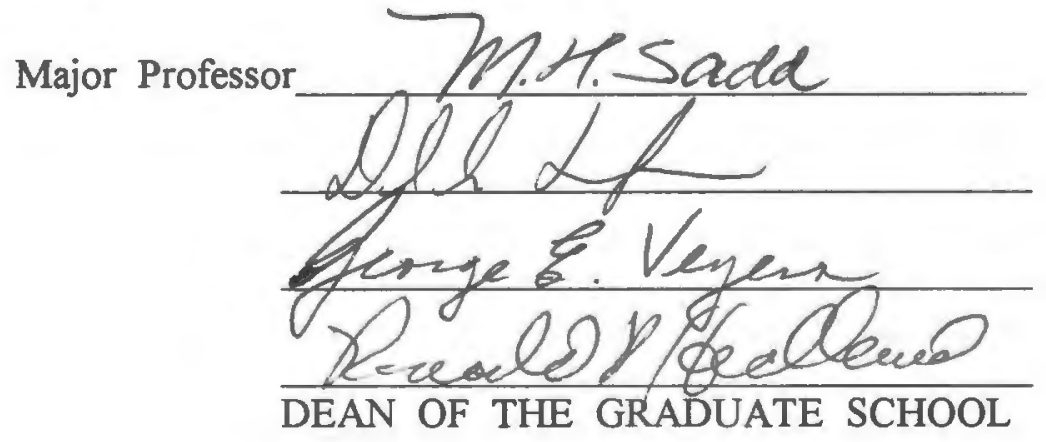




\section{ABSTRACT}

A numerical study of wave propagation in fluid saturated granular materials is presented. Because of their microstructure, granular materials are multi-phase, composed of solid particles along with pore fluid and/or gas. The mechanical response of such materials is quite complicated and difficult to model with classical continuum mechanics. Due to the microscopic heterogeneity of the particulate media, wave propagation in porous or granular materials is governed by complex microscopic physical behaviors which are sensitive to slight variations in fluid content or of the solid microstructure.

The numerical routine used to simulate the wave propagation processes was the discrete element method (DEM). Idealized particulate assemblies are used to model real granular materials. The DEM method uses the simplifying assumption of Newtonian rigidbody dynamics to calculate the translational and rotational motion of each particle in these model assemblies. A new normal contact law governing particle interactions through a fluid was developed based on the theory of elastohydrodynamics. Tangential interactions were accounted for by using a simple shearing approximation and a Coulomb law.

Using these new contact laws, simulations of one- and two-dimensional model assemblies were performed. Specific results for wave speed and amplitude attenuation are presented, and relationships are established between the microstructure or fabric of particulate materials and its macroscopic wave propagational behaviors. Results include 
the effects of particle size, wavelength, viscosity, interparticle gap spacing, etc. on dynamic load transfers in porous media.

It was observed that the presence of pore fluid can have significant effects on the macroscopic dynamic behaviors of particulate materials by changing the contact response between adjacent particles through hydrodynamic squeeze-film action. Wave attenuation increases and wave velocity decreases with increasing interparticle gap spacing, and these effects are more pronounced at small inter particle gap spacing. It was found that there was a slight increase in wave velocity with increasing pore fluid viscosity. Wave velocity was found to be higher through assemblies of stiffer particles. It also can be concluded that wave propagational behaviors of particulate materials are directly related to the microstructure or fabric of them. 


\section{ACKNOWLEDGMENTS}

First of all, I would like to extend my gratitude to my advisor, Dr. Martin H. Sadd, for his sincere guidance, invaluable help and financial support, without which, this work would have been impossible. A special thanks goes to my defense committee members Dr. George E. Veyera and Dr. David G. Taggart, and to committee chairman Dr. Armand J. Silva for serving on my committee and for their suggestions.

Also, I wish to thank Dr. Qiming Tai in particular for his-assistance in the initial stages of this work. I must also acknowledge Mr. Jim Byrnes for his technical help on the computer facilities at the Mechanical Engineering Computer Laboratory and making them available to me.

Bess Eaton Donuts deserves my thanks too for umpteen cups of coffee and donuts, which brought some lighter moments into my life and kept me going, even in the wee hours of morning.

I am highly indebted to my parents for their loving care and encouragement, and for being a constant source of inspiration to excel. 


\section{TABLE OF CONTENTS}

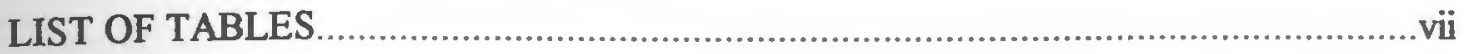

LIST OF FIGURES

1. INTRODUCTION

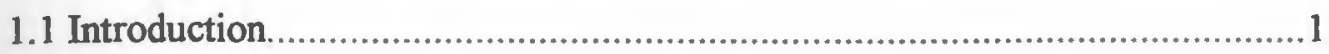

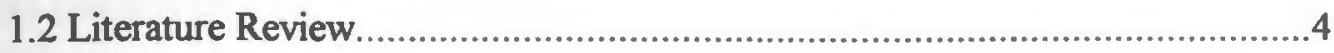

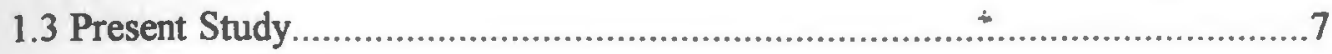

2. DISCRETE ELEMENT METHOD

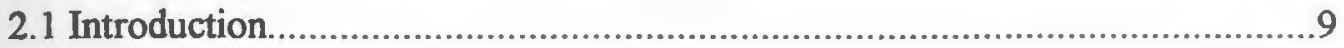

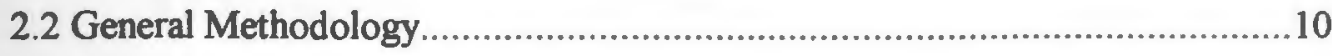

2.3 Basic Contact Laws...............................................................................11

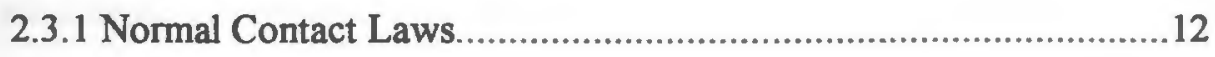

2.3.2 Tangential Contact Law............................................................16

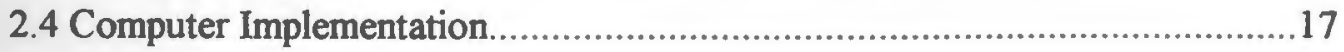

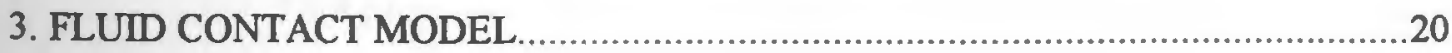

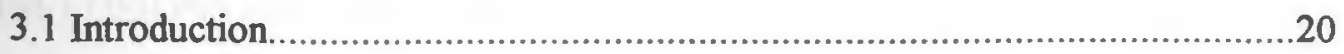

3.2 Elastohydrodynamic Contact Model........................................................20

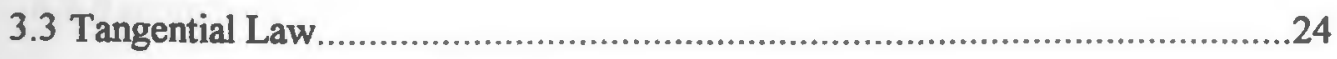

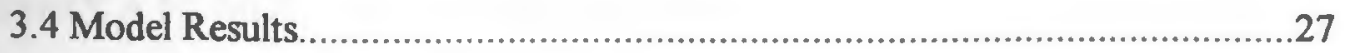

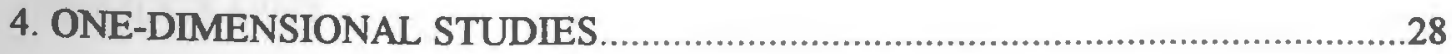

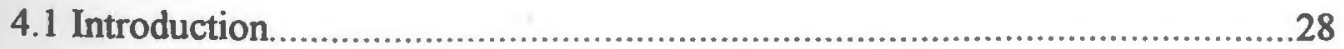


4.2.1 Effects of Gap, Viscosity and Particle Stiffness on

Wave Attenuation.

4.2.2 Effects of Viscosity, Gap Distance and Particle Stiffness on

Wave Speed. .30

4.2.3 Effects of Input Pulse Duration on Wave Attenuation. .30

5. TWO-DIMENSIONAL STUDIES.

5.1 Introduction.

5.2 Assembly Generators .38

5.2.1 Strongly Anisotropic Generator. .40

5.2.2 Moderately Anisotropic Generator 42

5.2.3 Weakly Anisotropic Generator. 43

5.3 Two-Dimensional Simulations Results. 45

5.3.1 Simulations Results for Strongly Anisotropic Assembly .46

5.3.2 Simulations Results for Moderately Anisotropic Assembly. .49

5.3.3 Simulations Results for Weakly Anisotropic Assembly 51

6. CONCLUSIONS AND RECOMMENDATIONS. .68

6.1 Conclusions. .68

6.2 Recommendations. .69

APPENDIX A NUMERICAL COMPUTER CODE. .71

BIBLIOGRAPHY .87 


\section{LIST OF TABLES}

\section{Chapter 5}

5.1 Parameters used in the numerical analysis of two-dimensional assemblies.

5.2 Simulations results for strongly anisotropic assembly

5.3 Simulations results for moderately anisotropic assembly

5.4 Simulations results for weakly anisotropic assembly 


\section{LIST OF FIGURES}

\section{Chapter 1}

1.1 Natural granular media.

\section{Chapter 2}

2.1 Particle normal contact laws.

2.2 A flowchart for the discrete element method computational techrique.

\section{Chapter 3}

3.1 Schematic of two normally approaching disks embedded in a viscous fluid

3.2 Shearing of fluid between two circular disks.

3.3 Plot of normal contact load vs. relative velocities for two circular disks.

\section{Chapter 4}

4.1 A single chain of circular disks.

4.2 Peak normal transmitted load vs. distance in a single straight chain.

4.3 Normal transmitted load vs. time in a single straight chain.

4.4 Normalized transmitted load vs. distance for different interparticle gap spacings.......33

4.5 Normalized transmitted load vs. distance for various pore fluid viscosities.

4.6 Normalized peak contact load vs. distance for different particle stiffnesses

4.7 Plot of wave velocity vs. interparticle gap spacing at different viscosities. 
4.8 Plot of wave velocity vs. particle stiffness

4.9 Contact load history at three contact points along the single chain for $\mathrm{T}=5 \mu \mathrm{s}$.

4.10 Contact load history at three contact points along the single chain for $\mathrm{T}=10 \mu \mathrm{s} \ldots \ldots .36$

4.11 Normalized peak contact loads vs. propagational distance for different input wave durations (dry case).

\section{Chapter 5}

Fig. 5.1 A random two-dimensional assembly

Fig. 5.2 Various fabric measures used for particulate materials.

Fig. 5.3 Strongly anisotropic media generator.

Fig. 5.4 Moderately anisotropic generator.

Fig. 5.5 Weakly anisotropic generator.

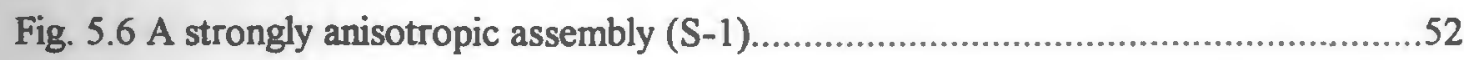

Fig. 5.7 Polar distribution plot for S-1 assembly ...............................................53

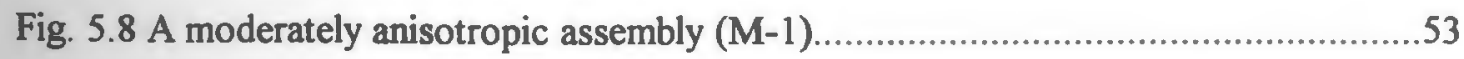

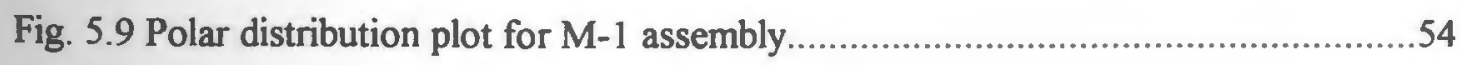

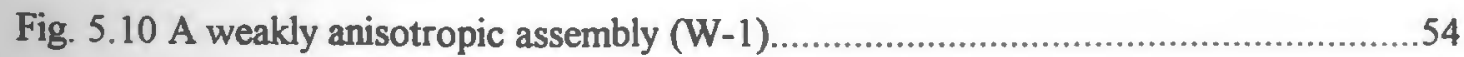

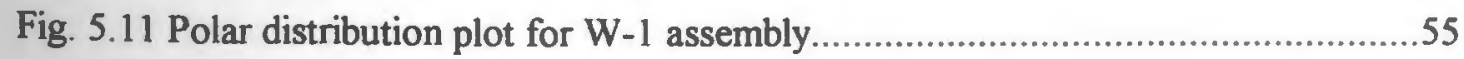

Fig. 5.12 Strongly anisotropic assembly (S-2) used for simulation ..............................55

Fig. 5.13 Polar plot of branch vector distribution for S-2 ........................................56

Fig. 5.14 Average normalized transmitted load in two directions for S-2 $\ldots \ldots \ldots \ldots \ldots \ldots \ldots . . . . .56$

Fig. 5.15 Vertical transmission vs. time at different interparticle spacings for S-2 2...........57 
Fig.5.16 Comparison of vertical transmission normalized w.r.t contact load at first layer and the input load

Fig. 5.17 Average normalized contact force for $5 \& 10 \mu$ s pulse durations for S-2 $2 \ldots \ldots \ldots . .58$

Fig. 5.18 Average normalized contact force for $5 \& 50 \mu$ s pulse durations for S-2..........58

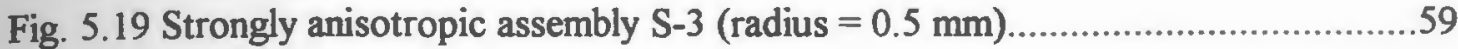

Fig. 5.20 Comparison of vertical transmission for assemblies with different sized particles

(constant input load).

Fig. 5.21 Comparison of vertical transmission for assemblies with different sized particles

(constant input stress).

Fig. 5.22 Moderately anisotropic assembly $M-2$ with particle radius $R=1 \mathrm{~mm}$ 60

Fig. 5.23 Polar plot of branch vector distribution for M-2 assembly .61

Fig. 5.24 Horizontal and vertical transmission through $\mathrm{M}-2$ 61

Fig. 5.25 Vertical transmission through M-2 for different interparticle gap spacing 62

Fig. 5.26 Horizontal transmission through M-2 for different interparticle gap spacing.....62

Fig. 5.27 Comparison of horizontal and vertical transmission for $\mathrm{M}-2(\mathrm{R}=0.5 \mathrm{~mm}) \ldots \ldots .63$

Fig. 5.28 Vertical transmission for assemblies with different sized particles. 63

Fig. 5.29 Horizontal transmission for assemblies with different sized particles. .64

Fig. 5.30 Weakly anisotropic assembly W-2 with particle radius $=1 \mathrm{~mm}$. .64

Fig. 5.31 Polar plot of branch vector distribution for W-2 assembly. .65

Fig. 5.32 Average transmitted load vs. time for horizontal and vertical directions for W-2 (interparticle gap spacing $=0.1 \mu \mathrm{m}$ ) 
Fig. 5.33 Average transmitted load vs. time in horizontal and vertical directions for W-2

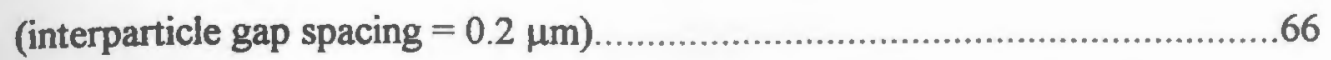

Fig. 5.34 Horizontal transmission for different interparticle spacings in W-2 ................66

Fig. 5.35 Vertical transmission for different interparticle spacings in $\mathrm{W}-2 \ldots \ldots \ldots \ldots \ldots \ldots \ldots . .67$

Fig. 5.36 Wave velocity / porosity relationship ......................................................67

\section{Appendix A}

Fig. A.1 Numerical computer code structure. 


\section{Chapter 1}

\section{INTRODUCTION}

\subsection{Introduction}

A granular or porous medium is an aggregate of solid elements (grains, matrix etc.) between which voids form a pore space (Fig. 1.1). The granules of the medium are free to displace from one another, and the voids may be filled with gas or fluids. These voids within a granular or porous medium give rise to the wide differences in physical behavior as compared with dense solids. Commonly granular/porous substances are complicated assemblages in which the presence of pore fluid, even in very small amounts, adds to the overall complexity of the material behavior.

Wave propagation in such discontinuous media has been of interest to the soil and rock mechanics community for many decades. The propagation of waves in the earth's crust is most intimately related to the properties of sand, soil and rocks. Such properties of these substances include the amount of pore water contained inside, particle size, shape and packing density, porosity, and any binding material which may be present.

Wave propagation through granular media is a complex phenomenon. It depends upon a number of parameters such as dynamic strain amplitude, static intergranular stress, material properties of individual grains, particle size, shape and orientation as well as boundary conditions. Apart form these variables, research has shown that the wave velocity and attenuation of stress wave propagation in granular medium is greatly influenced by the degree of saturation. Due to the microscopic heterogeneity, wave propagation in porous and granular geomaterials is governed by complex micro-processes which present considerable challenges in developing predictive models. It is a well known that in the dry granular medium, a mechanical signal is transmitted primarily through contact points between adjacent particles. But in the case of saturated granular medium, interparticle contact behaviors are significantly effected by the presence of pore fluid, and 

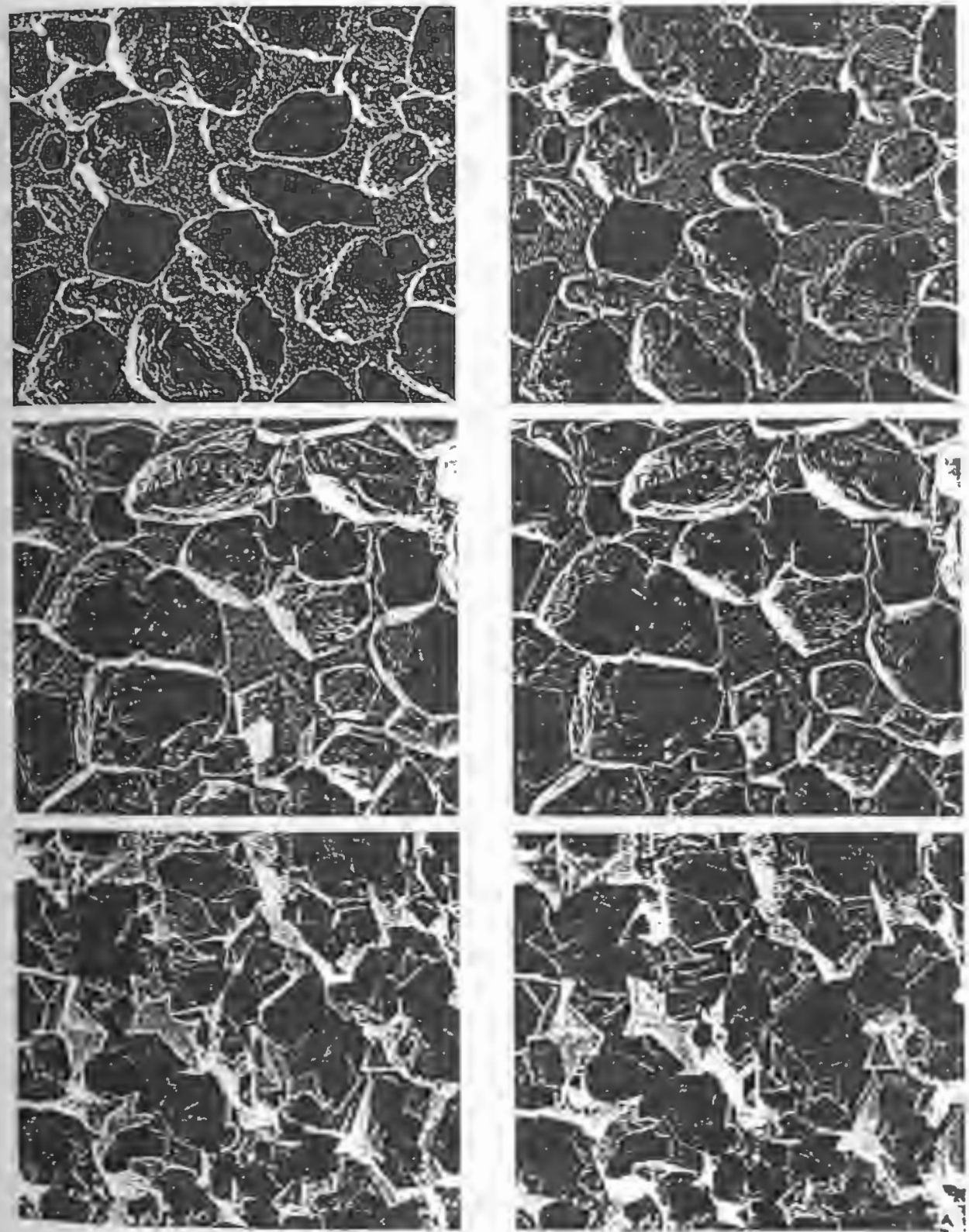

Fontainebleau sandstones

Fig. 1.1 Natural granular media (Bourbie et al., 1987) 
this produces sizeable differences in the dynamic material response when compared with dry, unbonded granular media. The presence of fluid occupying a portion of the pore space within the solid fraction requires a modeling strategy which incorporates the coupled mechanical response between the pore fluid and the solid skeleton.

According to Bourbie et al (1987), two approaches are possible for simulation of wave propagation in saturated porous media. One approach relies on the assumption that the concepts and principles of continuum mechanics can be applied to measurable macroscopic values, ignoring the microscopic level. Another modeling approach is based on the homogenization process, which helps to pass from microscopic to macroscopic behavior. The term microscopic is used here to apply to laws governing mechanisms at the scale of heterogeneity, whereas macroscopic laws refer to a scale related to the heterogeneous medium under study. Essentially, two homogenization methods exist.

One of them is based on an averaging process whereby a microscopic problem is first resolved at the elementary cell level containing an isolated heterogeneity (for example, a fluid-filled channel). From the solution to this elementary problem, the mean value of the cell of the quantity analyzed (stresses, strains, energies or relative fluid velocity) is inferred as a function of the macroscopic value imposed at the cell boundary (strains, stresses or relative flow velocity). The actual heterogeneous medium is then replaced by a fictitious homogeneous medium. The response of the latter to an imposed force is the mean value calculated previously. The function linking the actual medium with the fictitious one depends spatially on the geometric and mechanical parameters of the heterogeneities existing in the actual medium. The classic work by Biot (1962) used this method to characterize the flow of a fluid in a porous medium. This method is particularly effective for low and medium concentrations of heterogeneities.

A second homogenization method often used is based on the assumption of periodic repetition of the microscopic heterogeneous structure, imposing the periodicity of the solutions. The form of the macroscopic laws is obtained by making the spatial period 
tend towards zero with respect to the macroscopic scale. This method has the advantage of mathematical rigor, its systematic aspect, and its absence of concentration limits. Although these methods exist and have been successfully applied, many researchers have indicated that the complex microstructural nature of particulate material requires that a microstructural model be used to more accurately predict the mechanical behavior.

\subsection{Literature Review}

Research on the dynamic behavior and wave propagation through granular media dates back as early as the 1930's. Early attempts at modeling granular media involved simulations with regular arrays of elastic disks or spheres. The mechanical behavior of granular materials is greatly influenced by its microstructure. Iida (1939) was among the early pioneers of this field, who studied the velocity of wave propagation through assemblies of spheres using a microstructural approach based on simple mass-spring systems. Later, Takahashi and Sato (1949), Gassman (1951), Hughes and Cross (1951) and Hughes and Kelly (1952) conducted similar studies investigating the effect of saturation and pressure on the wave velocity. Brandt (1955) was the first to study the wave propagation through an irregular packing of particles. All these works neglected tangential interparticle contact forces. In reality, the contact forces arising due to the application of arbitrary external loadings are oblique to the contacting surfaces in a particulate media. Duffy and Mindlin (1957) proposed a simple model incorporating tangential forces into consideration for granular media.

Classic continuum mechanics laws of elastodynamics and their extensions including viscoelastic response have had limited success in predicting wave propagation in granular and porous materials. One of the more successful continuum mechanics modeling schemes occurred through the development of poroelasticity theory. Based largely on the remarkable efforts of Biot (1956a,b), poroelasticity theory is constructed on the concept of a coherent solid skeleton containing a fully connected, fluid filled pore space. Biot 
incorporated coupling between the fluid and solid phases with the solid behavior governed by an elasticity theory and the fluid motion modeled through a diffusion (Darcy) equation. The definition of pore pressure places some restrictions on the time scale at which the coupled diffusion-deformation processes can be analyzed. This occurs because the pore pressure must be locally equilibrated between the neighboring pores, and this length scale is linked to a time scale at through the fluid diffusion behavior. Thus in principle, Biot theory is limited to quasi-static processes; however, it has been applied by many researchers (eg. Stoll, 1989) to dynamic problems involving wave propagation and predictions of the theory have been experimentally verified by Plona (1982) and Ogushwitz (1985).

Modifications of Biot theory have included squirt flow mechanisms, which could be represented by a viscoelastic model, and such approaches have been developed by Mavko and Nur (1979), Palmer and Traviolia (1981), and Murphy et al. (1986) to theoretically model wave propagation through particulate media. These theoretical approaches involve modeling the pore fluid flow within idealized pore spaces. Most recently Dvorkin and Nur (1993) and Dvorkin et al. (1994) have combined squirt flow models with classical Biot theory and have developed a combined theory.

Another theoretical model used to describe wave propagation in porous and granular media is the distributed body theory developed by Goodman and Cowin (1972). It is based on the assumption that the medium is distributed in space by an independent kinematical function called the volume distribution function. This theory has been applied with some success to wave propagation problems by Nunziato et.al. (1977) and Sadd and Hossain (1988).

Further modeling work on granular materials has been based on the spatial arrangement of the solid particles and associated voids, commonly defined as fabric. Such fabric tensor models have been developed by Oda (1972), Nemat-Nasser (1982), and others. Additional theoretical models to describe granular media mechanics include 
endochronic theories (Bazant et al., 1983), pore-collapse mechanisms (Carroll et al., 1972), and statistical-stochastic approaches (Hudson, 1968; Endley and Peyrot, 1977).

Because granular media are aggregate assemblies of distinct particles interacting with one another primarily through contact mechanisms, a discrete modeling approach has been developed stemming from the field of molecular dynamics. It is a modeling strategy which uses Newtonian rigid-body mechanics to model the translational and rotational motion of particles in model material assemblies. Cundall and Strack (1979), and Kishino (1988) developed various types of discrete element methods. These methods were applied successfully to describe the behavior of granular materials under various loading conditions by Cundall and Strack (1979), Chang and Misra (1989), Thornton and Barnes (1986), Williams (1988), Rothenburg and Bathurst (1993), and Ting et al. (1989). Representative of some of this early research for the static response may be found in collected works by Satake and Jenkins (1982), Christoffeersen (1981), and Nemat-Nasser and Mehrabadi (1983). Numerical discrete element simulations of wave propagation in granular materials was first carried out by Sadd et al. (1989a). Sadd et al. (1989b) also studied the effects of inclusions and voids on wave propagation in granular materials. Tai (1993) used discrete element modeling to study wave propagation in unsaturated granular materials and he related his studies to the material's microstructure.Brown et al. (1980) studied the propagation of explosive shock wave through rubble screens using the DEM. Barbosa and Ghaboussi (1990) used this numerical technique for the static and dynamic large deformation analysis of multiple interacting deformable bodies. Cundall et al. (1978) developed a discrete element model of rock and soil structure. To model rock masses containing large fractures, Pan and Reed (1991) coupled the discrete element method with the finite element method. The finite element method has also been applied to study the wave motion in assemblies of particles. Sadd et al. (1991) replaced the particulate assembly with an equivalent network of elastic links to simulate elastic contact load transfer and analyzed the network using standard nonlinear finite element techniques. 
Some of the experimental work to investigate wave motion in granular materials have used the methods of photoelasticity and strain gage techniques. Drescher and De Josseling De Jong (1972) were the first to study the static behavior of granular materials using photoelasticity, and later their work was carried on by Drescher (1979) and Durelli and $\mathrm{Wu}$ (1983). Later more comprehensive photoelastic studies were conducted by Shukla and Rossamanith (1986), and Shukla and Damania (1987) for dynamic analysis of granular media. The experimental work was performed with the use of a high speed camera to record wave propagation through various assemblies of birefringent disks. Strain gage techniques have been applied by Shukla and Damania (1987) to stuđy wave motion in onedimensional model particulate materials. With regard to applications involving wave propagation problems, Shukla et al. (1988a, 1988b) and Sadd et al. (1993) have investigated the dynamic response of unsaturated particulate materials and DEM results have compared favorably with experimental measurements. Zhu et al. (1991) developed an xperimental-numerical hybrid technique to predict the intergranular contact load transfer in granular media subjected to explosive loading. These studies have shown that from a micromechanical point of view, load is transferred in a dry granular medium primarily through contact mechanisms between neighboring particles. Furthermore, for the dynamic case it has been observed that the propagation of mechanical waves through such a medium occurs along complex networks of paths determined by the material's granular microstructure. Thus, local microstructure or fabric plays an important role in the transmission of mechanical loadings through such materials, and the wave speed and amplitude can be related to material fabric.

\subsection{Present Study}

The present work is concerned with micro-mechanical modeling of wave propagation in saturated idealized granular materials, using the discrete element method. Proper modeling of the contact forces between adjacent particles through a thin film of 
fluid is essential for the correct application of this numerical technique, and the development of a new contact law will be described in Chapter 3. The local contact behavior can be thought of as the microscopic constitutive law for the material, and the developed contact law is based on elastohydrodynamic theory. The presence of pore fluid can have significant effects on the dynamic response of granular geomaterials through local fluid motion, viscous drag forces on particles and by changing the contact response between adjacent particles through squeeze-film elastohydrodymamic action. Based on this new contact law, a new computer code has been developed.

One-dimensional DEM computer simulations were conducted first in order to model wave propagation in a one-dimensional saturated granular material. These simulations were used for some initial studies of basic model predictions and alibration/determination of model parameters.

The main part of this thesis involved simulations of several large random twodimensional granular assemblies, which were generated with different anisotropy and fabric. Several runs were conducted to investigate horizontal and vertical wave motion in these random model materials, and an attempt was made to correlate the wave propagational characteristics such as wave speed and attenuation with the material's microstructure or fabric. 


\section{Chapter 2}

\section{THE DISCRETE ELEMENT METHOD}

\subsection{Introduction}

The discrete element method (DEM), which was originally developed by Cundall and Strack (1979), is a specific form of micromechanical modeling. It is a numerical technique capable of modeling the mechanical behavior of a large variety of particulate media. This computational method models the response of discontinuous materials by studying the behavior of individual idealized particles in assembly systems. In a dry granular medium, mechanical signals are transmitted primarily through contact mechanisms between neighboring particles. Due to its discrete nature, granular materials present much more complicated mechanical behavior under static and dynamic loadings than a continuum. Due to the inherent microscopic heterogeneity, wave propagation in porous and granular materials is governed by complex micro-processes which offer considerable challenges in developing predictive models. The term microscopic refers to behaviors occurring at the scale of heterogeneity; e.g. length scales of pore or particle size. The presence of fluid occupying a portion of the pore space within the solid fraction requires a modeling strategy which incorporates the coupled mechanical response between the pore fluid and solid skeleton. Therefore it is essential to study saturated granular media from the micro-level fabric view point.

The discrete element method has been used in recent years by several researchers to model the behavior of granular media subjected to static and dynamic loadings. Petrakis and Dobry (1988) used the distinct element method to model granular soils subjected to small strain anisotropic loading in which contact separation, sliding and rearrangement of the particles were allowed. Nelson et al. (1988) used this method to analyze the results of an experimental investigation into the inelastic behavior of geological material. DEM has also been applied to problems involving stress wave propagation in granular media 
Thornton and Bames (1986) used the DEM to investigate the mechanics of granular materials under quasi-equilibrium conditions. Shukla and Sadd (1990) have shown that numerical simulations using the discrete element method agree well with experimental wave velocity and amplitude attenuation data. Earlier, photoelasticity was used to observe static and dynamic load transfer processes in granular media by Drescher and De Josseling De Jong (1972), and Shukla and Rossmanith (1986). The drawback of this method is that the analysis of experimental data is time consuming for large assemblies of particles. Moreover, it is difficult to repeat identical experiments for materials with irregular microstructures. Another method which is used for simulating dynamic response of granular media is the finite element method. This approach, which was applied by Sadd et al. (1991), has used elastic network model. Finite element models however, are not best suited for this application as they take considerable amount of CPU time in calculating and updating the media stiffness matrix. This update requirement must be done to account for the new contacts coming into existence or disappearing during dynamic load transfer. The discrete element method appears to be ideally suited for numerical simulation of granular media. Many researchers have had success using this scheme and results have compared favorably with experimental data. Therefore the discrete element method was selected to study the dynamic response of saturated granular media in the present work.

\subsection{General Methodology}

To study the saturated granular media using the discrete element method, the material is assumed to be composed of large numbers of idealized particles. The numerical strategy uses Newtonian rigid-body dynamics to calculate the translational and rotational motion of each particle in these model assemblies. Contact laws between adjacent particles are constructed which serve to determine the contact force as a function of the relative displacement or the relative velocity between the particles. The technique establishes a discretized time stepping numerical routine, in which granule velocities and positions are 
obtained from numerical integration of the computed accelerations. It is assumed that during each time step, disturbances can not propagate from any particle further than its immediate neighbors. Under this assumption, the method becomes explicit, and therefore at any time increment the resultant forces (thus the accelerations) on any particle are determined solely by its immediate neighbor interactions. By using a force-displacement law, the interparticle force increments are calculated at all contacts from the relative positions and velocities of adjacent particles. The linear and angular accelerations of each particle are found by using Newton's laws. These accelerations are then integrated to find the new velocities and positions of all particles. This allows the calculations of new force increments and this process is repeated for each time step.

For applications to wave propagation, the movement of the individual particles are a result of the propagation through the medium of disturbances originating at particular input loading points. Consequently, the wave speed and amplitude attenuation (intergranular contact force) will be functions of the physical properties of the discrete medium, i.e. the microstructure. In order to model actual particulate materials, the method is typically applied to granular systems containing large number of idealized particles (e.g. circular disks or spheres) in regular or random packing geometries. Such model materials can be computationally generated with varying degrees of microstructural fabric.

\subsection{Basic Contact Laws}

In a discontinuous medium, internal forces arise due to the existence of contacts or intergranular interactions between the particles that comprise the material system. These contacts can be classified as either soft or hard. The contact is called soft when the contact force can be expressed as a function of particle deformation or displacement. A good example of this type of contact is Hertz contact. The other type of contact, in which particles are only briefly in contact is called hard contact. For this case, there is instantaneous momentum exchange due to the brief collisions, and the contact forces can 
be calculated using momentum balance. This theoretical model is generally applied to the simulation of granular flows or molecular dynamics where particles are moving at high speeds and interacting with one another through collisions.

Soft contact laws may be further classified as normal or tangential with respect to the contact surface. For the static case the normal contact law can be determined by Hertz contact theory or from experiments. However, it is difficult to determine the contact law for the dynamic case due to the wave motion effects. In general, contact laws between the $\mathrm{i}^{\text {th }}$ and $\mathrm{j}^{\text {th }}$ particles can be expressed as two equations signifying normal and tangential contact forces by

$$
\begin{aligned}
& F_{n}^{(i j)}=F_{n}^{(i j)}\left(\delta_{n}^{(i j)}, v_{n}^{(i j)}, \delta_{t}^{(i j)}, v_{t}^{(i j)}\right) \\
& F_{t}^{(i j)}=F_{t}^{(i j)}\left(\delta_{n}^{(i j)}, v_{n}^{(i j)}, \delta_{t}^{(i j)}, v_{t}^{(i j)}\right)
\end{aligned}
$$

where $\delta_{n}{ }^{(i j)}$ and $\delta_{t}(i j)$ are the normal and tangential deformations of particle $i$ with respect to particle $\mathrm{j}$, and $v_{\mathrm{n}}(\mathrm{ij})$ and $v_{\mathrm{i}}^{(\mathrm{ij})}$ are the normal and tangential velocities of $\mathrm{i}$ relative to $\mathrm{j}$. The contact forces may also be history dependent.

Sof contacts are the most appropriate for our study, and the following sections briefly describe different types of soft contact laws.

\subsubsection{Normal Contact Laws}

The normal contact law can be determined by Hertz contact theory or from experiments for the static case. But this approach has to be modified for the dynamic case in which the load transfer is of short duration. In general, the normal contact law given by Eq. $(2.1)_{1}$ represents a variety of cases including both displacement and velocitydependent contact forces. Velocity dependent damping acting like dashpots produces energy dissipation which is a needed ingredient in modeling real granular materials. Two forms of such damping were introduced by Sadd et al. (1989): a local damping 
proportional to the relative disks velocities, and a global damping proportional to the absolute velocities. In the same manner, contact laws depending only upon relative particle displacements can also lead to energy dissipation. Basically, two general aspects which must be incorporated in an appropriate contact constitutive law are the correct stiffness (to be able to model the proper wave velocities) and the correct dissipation mechanisms (to predict wave attenuation). Fig. 2.1(a)-(c) shows three specific types of normal contact laws.

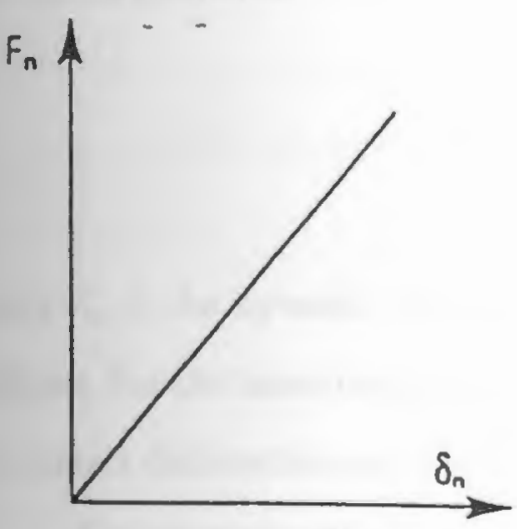

(a) Linear normal contact law

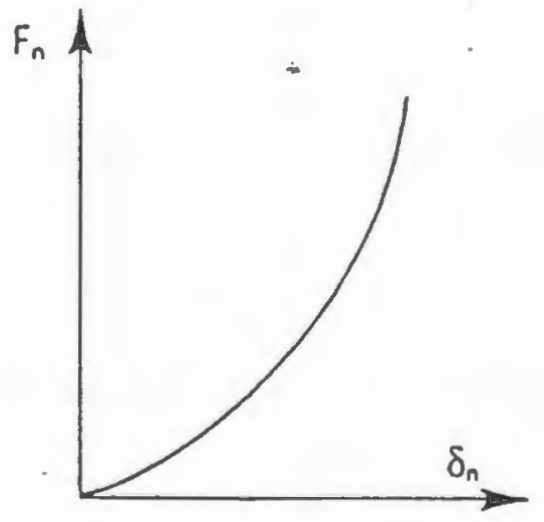

(b) Nonlinear notmal contact law

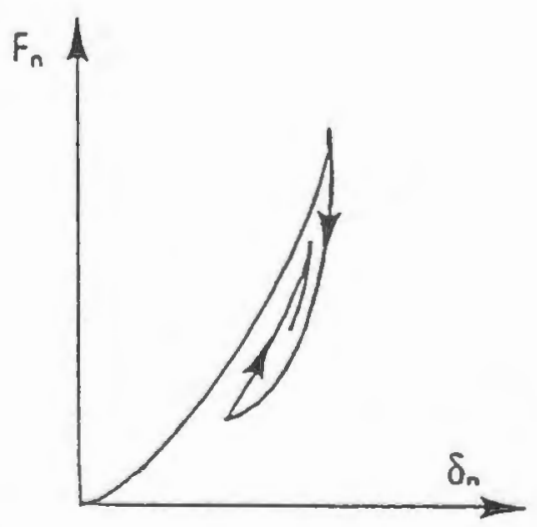

(c) Nonlinear-hysteretic normal contact law

Fig. 2.1 Particle normal contact laws (Tai, 1994) 


\section{i. linear normal contact law}

The linear normal contact law is based upon two assumptions: 1) no out of plane deformations occur, and 2) plane strain conditions exist. Based on these assumptions, application of Hertz contact theory yields a static linear contact law between the forces and the relative displacements of two circular disks in contact (see Fig. 2.1a). The dynamic contact stiffness may be scaled from the static Hertz value, and hence the normal contact law can be written as

$$
F_{n}=K_{n} \delta_{n}=\alpha K_{n}^{(s)} \delta_{n} ; \quad \alpha<1
$$

where $K_{n}$ is the dynamic stiffness, $\alpha$ is a stiffness ratio, and $K^{(s)}{ }_{n}$ is the static contact stiffness. For the linear contact law, these stiffnesses are constant and are independent of the contact deformation and history.

To incorporate this law with the discrete element method, some form of local or global damping must be included in the model. The local damping following a simple linear law (linear viscous dashpot) has been used and global damping is neglected for the dry granular materials.

The drawback of this law is that it produces a very unrealistic wave form dispersion, which is not consistent with experimental data of real materials.

\section{ii. nonlinear contact law}

This law was developed by Sadd et al. (1992) to eliminate the wave dispersion observed in the linear normal contact law. The linear law does not incorporate the effect of changes in particle shape. Instead, it is only concerned with deformation at the contact points. According to Johnson (1985) the relative displacement between the disks cannot 
be determined by only considering the deformation near the contact point. Johnson, using a superposition of Hertz theory, a uniform stress field, and a point-loaded disk problem, derived a simplified solution for a nonlinear relationship between the contact force $F_{n}$ and the center-point displacement $\delta_{n}$ at the contacts given by

$$
F_{n}=\alpha_{o} \delta_{n}^{p}
$$

where $\alpha_{0}$ and $p$ are model parameters determined by comparison of wave velocities obtained from the model with those found experimentally. Generally $\mathrm{p}>1$, thus providing a stiffness that increases with load (see Fig. 2.1b). As with the case of linear contact law, this law too does not fully correct the unreasonable predictions of wave form dispersion.

\section{iii. nonlinear hysteretic normal contact law}

Sadd et al. (1992) showed that the linear and nonlinear contact laws with linear velocity dependent damping mechanism over predict the wave form dispersion measured in experiments on granular materials. Apart from that, the velocity dependent damping indicates some form of viscous mechanism present in the material, which does not match with the physical evidence for dry granular materials. Consequently, Sadd et al. (1992) proposed a deformation dependent damping through a nonlinear hysteretic law accounting for different loading and unloading behaviors (see Fig. 2.1c) which is given as

$$
\begin{aligned}
& F_{n L}=\alpha_{L} \delta_{n}^{p} \ldots \ldots \ldots \ldots \ldots \ldots \ldots \ldots . . . \ldots \ldots \text { loading } \\
& F_{n U}=\alpha_{U} \delta_{n}^{p+q} \ldots \ldots \ldots \ldots \ldots \ldots . . . . . . \text { umloading } \\
& F_{n r L}=\beta F_{n L}+(1-\beta) F_{n U} \ldots . . . . . . \text {. reloading }
\end{aligned}
$$

Here, the value of $q$ is determined by 


$$
\mathrm{q}=\left(A \delta_{1, \text { max }}\right)^{r}
$$

where $A$ and $r$ are model constants, and $\delta_{n, m a x}$ is the value of $\delta_{n}$ when the normal contact force has reached its maximum value. $\beta$ is defined as

$$
\beta=\frac{\delta_{\mathrm{n}}-\delta_{\mathrm{n}, \min }}{\delta_{\mathrm{n}, \max }-\delta_{\mathrm{n}, \mathrm{min}}}
$$

where $\delta_{n}$ is the current relative displacement at the contact and $\delta_{n, m i n}$ the minimum value of it in the previous reloading or unloading path. When reloading begins $\delta_{n}$ equals $\delta_{n, \min }$ and $\beta$ equals zero. Clearly, $\beta$ has its maximum value, which is one, when $\delta_{n}$ equals $\delta_{n, m a x}$ which indicates the beginning of new loading. The values of $\alpha_{\mathrm{L}}, \mathrm{p}, \mathrm{A}$ and $\mathrm{r}$ are related to the material and geometric properties of the particles and are determined from experimental calibration tests. However, the parameter $\alpha_{U}$ is selected to make the unloading path initiate at the peak point of loading.

\subsubsection{Tangential Contact Law}

The tangential contact behavior is complicated to model as it may depend upon the amplitude of the normal contact force and be history dependent. Moreover, there could be partial slip within the contact area of the particles for the dry case, and between fluid and the particle surface in the case of saturated granular materials. Tai (1994) used a tangential contact law proposed by Walton et al. (1991) with some modifications to model the dry case. The basic concept which was incorporated into that law is that the effective tangential contact stiffness decreases with an increase of the tangential force, and goes to zero when full sliding occurs. 
For the present study of the saturated granular materials, a new tangential law is developed, which will be described later in chapter 3.

\subsection{Computer Implementation}

Simulation of wave propagation and dynamic load transfer in saturated granular media is accomplished using the discrete element method by discretizing the time period into a series of time steps. It is assumed that by making these increments small enough, disturbances within a particle can at most only propagate to its neighboring particles within a given time step.

In order to describe the process, consider the case of two typical disks (say $i$ and $j$ ) in contact as shown in Fig. 2.2. The position, velocity, acceleration, angular velocity, angular acceleration, radius and mass of disk $\mathrm{i}$ are labeled as $\mathrm{r}_{\mathrm{i}}, \mathrm{v}_{\mathrm{i}}, \omega_{\mathrm{i}}, \alpha_{\mathrm{i}}, \mathbf{R}_{\mathrm{i}}$ and $\boldsymbol{m}_{i}$, with similar notations for disks $\mathbf{j}$. The unit normal vector $\mathbf{n}$ and unit tangential vector $\mathbf{t}$ are defined as shown, and these establish the normal and tangential directions used for contact analysis.

Using Newton's second law we can calculate the translational and angular accelerations $\ddot{x}_{i}$ and $\ddot{\theta}_{i}$ for the particle $i$ as follows

$$
\begin{aligned}
& \sum_{j=1, i \neq j}^{N} F^{(i j)}+\sum_{k=1}^{s} F_{i}^{(k)}=m_{i} \ddot{x}_{i} \\
& \sum_{j=1, i \neq j}^{N} M^{(i j)}+\sum_{k=1}^{s} M_{i}^{(k)}=I_{i} \ddot{\theta}_{i}
\end{aligned}
$$

where $F^{(i j)}$ and $M^{(i j)}$ are the interparticle contact forces and moments acting on the particle $\mathrm{i}$ due to the interactions with the neighboring particles, and $F_{\mathrm{i}}^{(\mathrm{k})}$ and $M_{\mathrm{i}}^{(\mathrm{k})}$ are the noninterparticle forces (body forces or internal forces) and moments respectively. $\mathrm{N}$ is the number of particles interacting with one another, and $s$ the number of internal forces or moments acting on the particle $\mathrm{i}$. 
Particle translational and angular velocity increments are calculated using a simple forward difference formula as follows

$$
\begin{aligned}
\Delta v_{i} & =\ddot{x}_{i} \Delta t \\
\Delta \dot{\theta}_{i} & =\ddot{\theta}_{i} \Delta t
\end{aligned}
$$

The normal component of relative velocity between the two disks is given by

$$
v_{n}^{(i j)}=\left(v_{i}-v_{j}\right) \cdot n^{(i j)}
$$

while the tangential relative velocity is

$$
v_{i}^{(i j)}=\left(v_{i}-v_{j}\right) \cdot t^{(i j)}-\left(\dot{\theta}_{i} R_{i}+\dot{\theta}_{j} R_{j}\right)
$$

Using a finite difference scheme with constant properties over the time interval, the relative velocities may be integrated with respect to time to yield the incremental relative normal and tangential displacements, i.e.

$$
\begin{aligned}
& \Delta \delta_{n}^{(i j)}=v_{n}^{(i j)} \Delta t=\left[\left(v_{i}-v_{j}\right) \cdot n^{(i j)}\right] \Delta t \\
& \Delta \delta_{t}^{(i j)}=v_{t}^{(i j)} \Delta t=\left[\left(v_{i}-v_{j}\right) \cdot t^{(i j)}-\left(\dot{\theta}_{i} R_{i}-\dot{\theta}_{j} R_{j}\right)\right] \Delta t
\end{aligned}
$$

These new relative normal and tangential displacements are in turn used to update the particle locations and this determines new contact forces using an appropriate contact Jee-displacement law. The new contact forces provide the new values of accelerations, velocities and displacements in the next following time step. This procedure is extended to every particle in the particulate medium. In this manner, large assemblies of disks can be analyzed in a reasonable amount of computer time. A computer flowchart of this discrete element method technique is given in Fig. 2.2. 


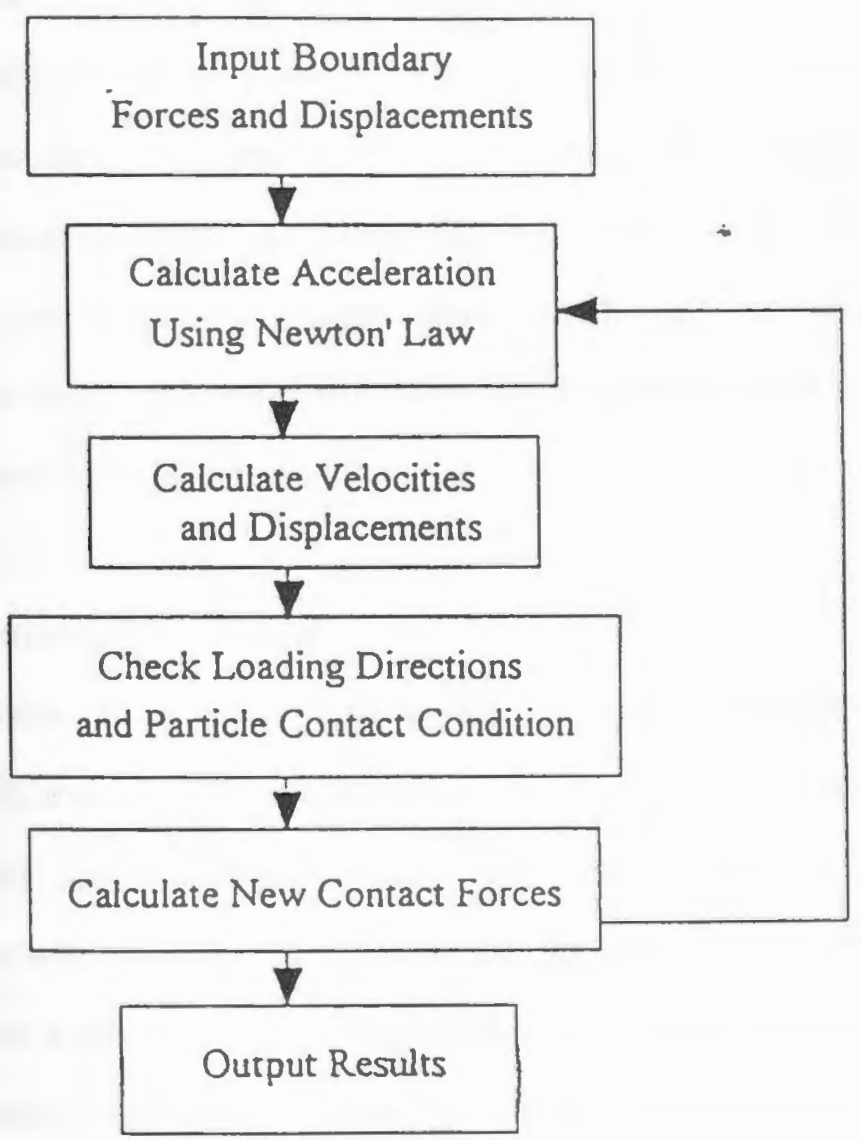

Fig. 2.2 A flowchart for the discrete element method computational technique 


\section{Chapter 3 \\ FLUID CONTACT MODEL}

\subsection{Introduction}

As mentioned earlier, the presence of pore fluid will create several new forces at the particulate level, and this requires the development of new contact laws governing particle interactions through a fluid. For fully saturated conditions, the capillary forces would vanish, whereas the phenomena of viscous drag and squeeze-film contact lubrication would produce sizeable particulate forces. Viscous drag effects would be predominant for large interparticle spacings where neighboring interaction forces are negligible. On the other hand, squeeze-film contact forces would be the most significant for dense packings of particulate systems.

\subsection{Elastohydrodynamic Contact Model}

The contact force for such a problem can be modeled using the theory of Hastohydrodynamics (Cameron, 1966 and Gohar, 1988). Consider the case of two particles (approximated as two circular disks) embedded in a viscous fluid, and approaching each other with a relative normal velocity $V(t)$, see Fig. 3.1. The fluid film in the contact zone will be squeezed, and a sizable pressure is thus built up in this contact region. The fluid pressure distribution in the gap will exert a loading on each particle surface, and this loading is sufficient in certain cases to produce significant particle deformation. In this fashion, the micro-contact law governing particle interactions for saturated granular materials involves the complication of coupling both fluid and solid behavior, and this creates significant differences from behaviors found in the dry case. Past studies of this elastohydrodynamic problem have been carried out by Christensen (1961), Kerrebrugh (1970) and Lee and Cheng (1973). In these studies, very elaborate and complicated solutions methods to determine the fluid pressures and deformation profiles 
between converging circular cylinders or disks were developed. Solutions were commonly formulated as integral equations, and numerical evaluations normally used some type of iteration strategy:

The problem geometry is shown in Fig. 3.1 where the distance between particle surfaces is denoted by $h(x, t)$, a function of coordinate $x$ and time $t$, and $\delta(x, t)$ is the particle deformation.

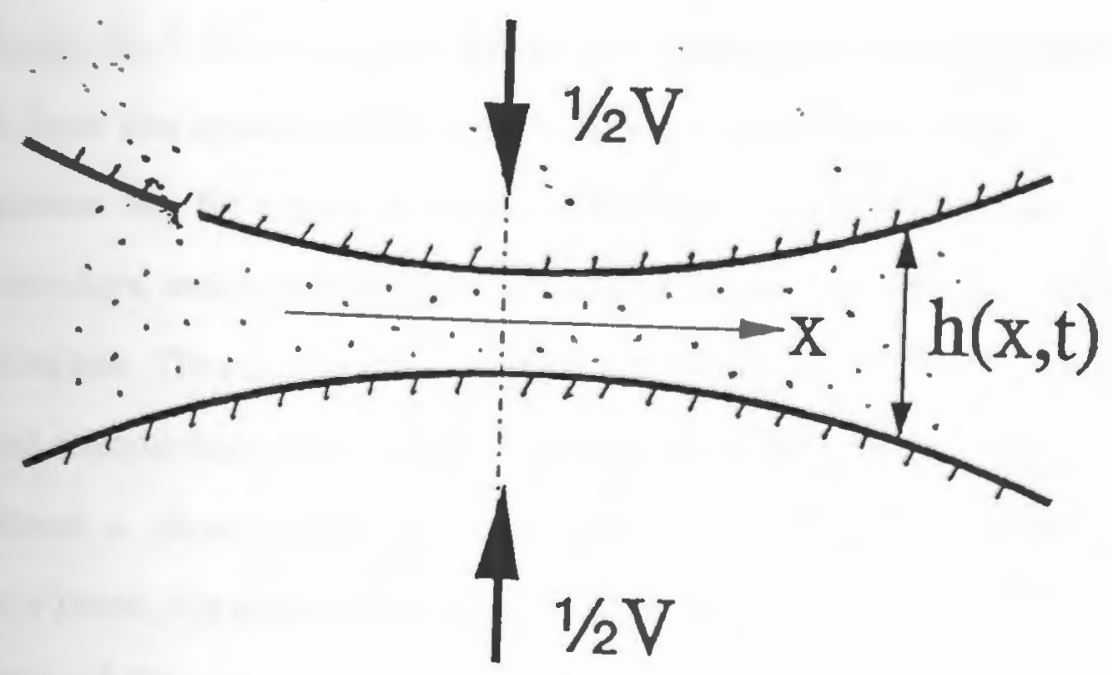

Fig. 3.1 Schematic of two normally approaching disks embedded in a viscous fluid 
This problem is governed by the Reynolds equation for two-dimensional flow, given by

$$
\frac{\partial}{\partial x}\left[\frac{\rho h^{3}}{\eta} \frac{\partial p}{\partial x}\right]+\frac{\partial}{\partial y}\left[\frac{\rho h^{3}}{\eta} \frac{\partial p}{\partial y}\right]=6\left\{U \frac{\partial}{\partial x}(\rho h)+V \frac{\partial}{\partial y}(\rho h)+2 \rho\left(w_{1}-w_{2}\right)+2 h\left[\frac{d \rho}{d t}\right]\right\}
$$

Assuming mass density $\rho$ and viscosity of the fluid $\eta$ to be constant with flow only in the $x$ direction, the fluid pressure $p(x, t)$ can be related to $h(x, t)$ by the reduced Reynolds equation

$$
\frac{\partial}{\partial x}\left(h^{3} \frac{\partial p}{\partial x}\right)=12 \eta \frac{\partial h}{\partial t}
$$

Since $h(x, t)$ can include particle deformation $\delta(x, t)$ which is determined by elasticity theory, Eq. 3.2 is a coupled relation, and typically an iterative method would be required to solve this equation for the pressure distribution $p(x, t)$. Incorporating such a numerical scheme into the discrete element method would result in a very computationally intensive procedure, and would therefore result in unreasonable amount of CPU time to run the simulations. Thus a simplified alternative procedure is developed in order to avoid the numerical complexities. This simplified approach first assumes that the particle is rigid, and this allows a closed-form solution for $p(x, 1)$ from Eq. 3.2. Once this pressure distribution is found, the particle deformation can be calculated from simple Hertz contact stress theory, and this new particle shape may be then used to calculate a new pressure distribution. Thus initially let $\delta(x, t)=0$, and the undeformed particle surface can be approximated by the relation

$$
h(x, t) \approx h(0, t)+\frac{x^{2}}{2 R^{*}}
$$


where $R^{*}$ is the reduced radius related to particle radii $R_{I}$ and $R_{2}$ by

$$
\frac{1}{R^{*}}=\frac{1}{R_{1}}+\frac{1}{R_{2}}
$$

The solution to the Eq. 3.2 for this case is found to be

$$
p(x, t)=-6 R * \eta \frac{V(t)}{h^{2}(x, t)}
$$

With the pressure distribution known, the total contact load between the particles is given by integrating the pressure, i.e. $F=\mathrm{H} \int p \mathrm{dx}$, where $\mathrm{H}$ is the disk thickness. Carrying out the integration over the disk surface yields

$$
F(t)=3 \sqrt{2} \pi \eta H\left(\frac{R^{*}}{h(0, t)}\right)^{3 / 2} V(t)
$$

In order to include the effects of particle deformation, a combined model with two series-connected stiffness (spring) elements are used. One spring element represents the fluid stiffness while the other one includes the solid (particle) stiffness resulting from the elastic deformation within the particle. The pressure loading will produce deformations such that the distance between particle surfaces will change from $h$ to $h(x, t)+\delta(x, t)$, where $\delta(x, t)$ is the contact deformation for the dry case. If the disk shape is assumed to be unchanged, then the new load $F(t)$ is still given by Eq. 3.6 using the modified value of $h(x, t)$, i.e.

$$
F(t)=3 \sqrt{2} \pi \eta H\left(\frac{R^{*}}{h(0, t)+\delta}\right)^{3 / 2} V(t)
$$


In dry granular materials, it has been shown by Sadd et al. (1992) that the normal contact force $F$ can be related to contact deformation by the relation $F=\mathrm{K} \delta^{1.4}$, where $\mathrm{K}$ is a material stiffness constant. The forces in the fluid and solid springs should be equal, and this leads to the result

$$
3 \sqrt{2} \pi \eta \mathrm{H}\left(\frac{\mathrm{R}^{*}}{\mathrm{~h}(0, \mathrm{t})+\delta}\right)^{3 / 2} \mathrm{~V}(\mathrm{t})=\mathrm{K} \delta^{1.5}
$$

where on the right-hand side, the exponent on $\delta$ has been modified to the value of 1.5 to avoid computational complexities. Solving Eq. 3.8 for $\delta$ and substituting the result into Eq. 3.7 yields

$$
F(t)=\frac{C_{1} V(t)}{\left(0.5\left(h(0, t)+\sqrt{h^{2}(0, t)+4\left(\frac{C_{1}}{K}\right)^{2 / 3}}\right)\right)^{3 / 2}}
$$

where $C_{1}=3 \sqrt{2} \eta \pi R^{* 3 / 2}$ H. Eq. 3.9 then gives the normal contact force when two disks approach each other. For the case when the two particles move apart, the contact law governed by Eq. 3.9 is not suitable since a negative velocity $V$ may lead to a negative value within the square root. For this case, the solid contact law is used.

\subsection{Tangential Law}

The tangential contact behavior of saturated granular media is difficult to model rigorously, and thus a simplified modeling scheme is used based upon the simple shearing of a Newtonian fluid within the small inter-particle gap as shown in Fig. 3.2. If the particle surfaces are completely separated, the fluid film usually behaves as Newtonian and there is 
no slip between the solid and the fluid. Since we are neglecting the inertia forces, the fluid film can be treated as a case of viscous flow in which pressure and viscous forces are everywhere in balance. If the gap is small then the fluid motion may be approximated by a linearly varying distribution (see Fig. 3.2), and the resulting shear force between the particles is then given by

$$
F_{t}=\eta A \frac{V_{1}-V_{2}}{h}
$$

where $F_{i}$ is the shearing or tangential force, $A$ is the effective area of the gap zone, $\eta$ is the fluid viscosity, $V_{1}$ is the absolute tangential velocity of particle $1, V_{2}$ is the absolute tangential velocity of particle 2 , and $\mathrm{h}$ is the minimum distance between paticles 1 and 2 .

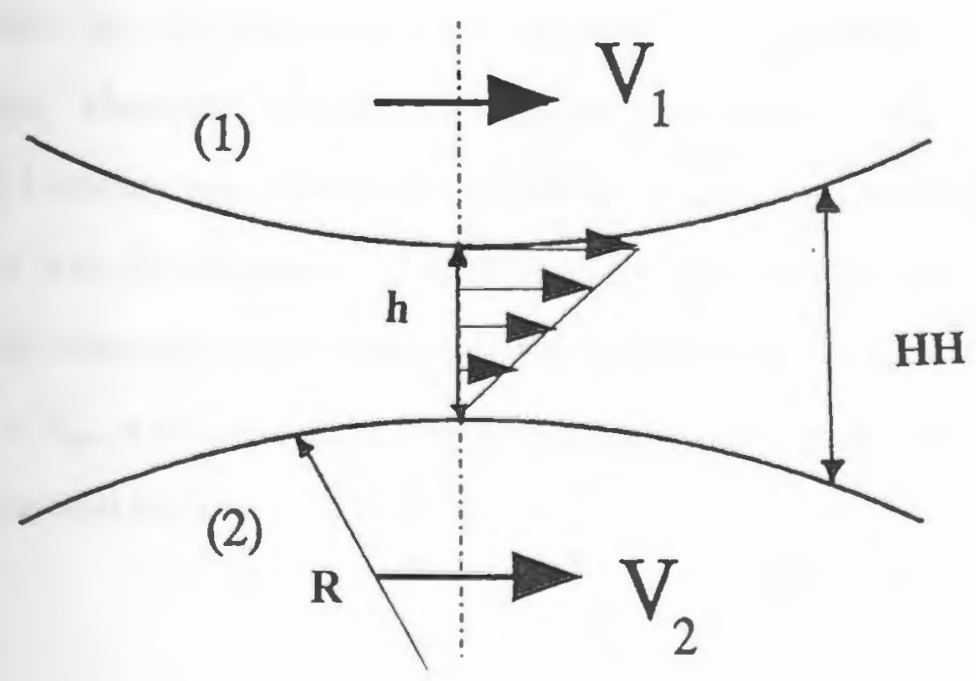

Fig. 3.2 Shearing of fluid between two circular disks 
The effective area $A$ is related to particle thickness $H$, particle radius $R$, and the maximum distance between the particles inside the influence or effective zone $\mathrm{HH}$ as follows (see Fig. 3.2)

$$
A=2 * H \sqrt{(H H-h) R-\left(\frac{H H-h}{2}\right)^{2}}
$$

As the particle spacing $\mathrm{h}$ becomes very small (of the order of a micrometer), relation (3.10) breaks down and predicts unrealistically high shear stresses. For this case it is well known that the no-slip condition of classical-fluid mechanics should be modified to include a tangential slip velocity along neighboring particle surfaces. This acts to soften the unrealistically high velocity gradient. This phenomenon is more dominant in the case of gaseous lubrication. It was suggested that for liquids, when the particle spacing becomes very small, often of molecular proportions, then the friction characteristics of the lubricated contact are determined by the properties of the surface layers and the underlying solids. Moreover, the viscosity of the lubricant has little effect on the performance of boundary lubricated contacts and the frictional behavior broadly follows the well-known laws for unlubricated surfaces. This idea was incorporated into our modeling scheme when the particle spacing $h$ goes below a certain critical value $h_{c r}$ So, for the case $h<h_{c r}$, a simple friction law between two contacting surfaces is used to calculate the tangential force as follows

$$
\mathrm{F}_{1}=\mu \mathrm{F}_{\|}
$$

where $F_{t}$ is the tangential force, $\mu$ is the coefficient of dynamic friction, and $F_{n}$ is the normal contact force between the two generic particles. $\mu$ is calculated by equating Eqqus. 3.10 and 3.12 for $h=h_{c r}$. 


\subsection{Model Result}

To check the characteristics and consistencies of the fluid contact model developed for dynamic load transfer in saturated granular media, a plot of normal contact load $F$ versus relative normal particle velocity $V$ for a pair of generic particles was constructed using Eq. 3.9 and is shown in Fig. 3.3. The values of different parameters used for calculating the normal contact load at different relative normal velocities of particles for given particle stiffnesses are $\eta=0.001 \mathrm{Ns} / \mathrm{m}^{2}, \mathrm{R}=1.0 \mathrm{~mm}, \mathrm{H}=1.0 \mathrm{~mm}$, and $h(0, t)=0.1 \mu \mathrm{m}$.

It is evident from Fig. 3.3 that normal contact load increases non-linearly with the relative normal particle velocity. Also no force is transmitted when the relative normal velocity is zero. Moreover particles with high stiffness impart more load as compared to particles with low stiffness.

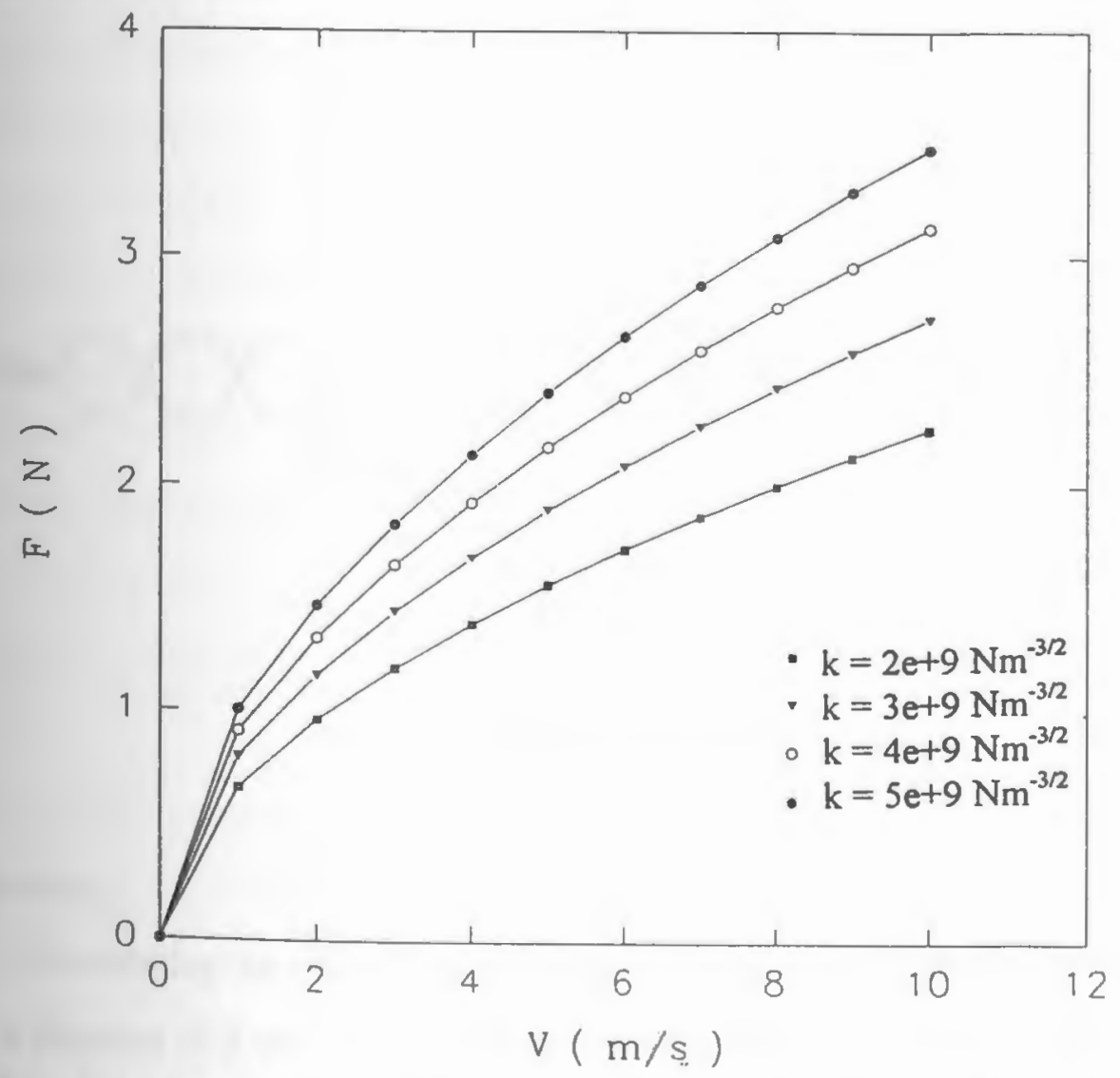

Fig. 3.3 Plot of normal contact load vs. relative velocities for two circular disks 


\section{Chapter 4 \\ ONE-DIMENSIONAL STUDIES}

\subsection{Introduction}

With the previously described fluid contact model incorporated in the discrete element computer code, numerical experiments were conducted in order to simulate wave propagation in a one-dimensional saturated granular material simulated by a single chain of circular disks as shown in Fig. 4.1. These simulations are used for some initial studies of basic model predictions and calibration/determination of model parameters. These studies are later helpful in the simulations of wave propagation through two-dimensional granular assemblies.

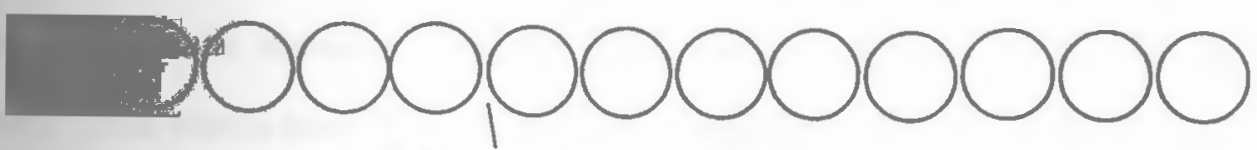

Fluid Filled Gaps

Fig. 4.1 A single chain of circular disks

\subsection{Results}

Considering the modeling of a coarse sand, the circular particles were assumed to have a diameter of $2 \mathrm{~mm}$, thickness of $1 \mathrm{~mm}$ and to be of quartz with a density of 2650 $\mathrm{kg} / \mathrm{m}^{3}$. These values were selected on the basis of data collected for real granular 
materials. The simulations incorporated different interparticle gap openings (of the order of $10^{-7} \mathrm{~m}$ ) between neighboring particles, and the model system was assumed to be completely saturated with water of viscosity of $0.001 \mathrm{Ns} / \mathrm{m}^{2}$. The input loading applied to one end of the chain of particles was taken to be a triangular time dependent pulse of duration of $5 \mu \mathrm{s}$ with a peak value of $2 \mathrm{~N}$ to simulate a wave front pressure of $10^{6} \mathrm{~N} / \mathrm{m}^{2}$ (i.e. 10 bar).

Using these parameters, a computer simulation was run and the wave attenuation or intergranular contact force results are shown in Fig. 4.2. Attenuation is a parameter, which characterizes the loss of energy undergone by a wave đuring its propagation through a given media or material. Like velocity, it depends upon number of factors such as pressure, particle size, interparticle gap spacing, pore fluid viscosity etc. It can be observed that the attenuation is quite severe during the passage through the first several particles near the point of input loading, but then the attenuation rate decreases with the propagational distance. The wave speed for this case was found to be approximately 1800 $\mathrm{m} / \mathrm{s}$. Another run was made with a different input peak loading of $4 \mathrm{~N}$ to check the consistency of the model, which is in the range of wave speeds found through real saturated granular materials. Fig. 4.3 gives the contact load profiles of every fourth contact point versus time. The wave speed in this case was found to be around $1860 \mathrm{~m} / \mathrm{s}$, and the duration of contact load at each contact point is about $5 \mu \mathrm{s}$, which gives the ratio of wavelength to disk diameter as 4.65 . The value of the material stiffness $k$ was taken as $5 \times 10^{10} \mathrm{Nm}^{-3 / 2}$ for both cases.

\subsubsection{Effect of Gap, Viscosity and Particle Stiffness on Wave Attenuation}

The effects of interparticle gap spacing, pore fluid viscosity and particle stiffness on one-dimensional wave propagation were also investigated and the results are shown in the Figs. 4.4, 4.5 and 4.6 , respectively. Fig. 4.4 clearly shows that wave attenuation through first few particles is much higher for larger gap distances between the particles. 
As the propagational distance increases, the attenuation difference became negligible. Although gap distance has significant effect on wave attenuation, pore fluid viscosity has a lesser influence, as can be seen from Fig. 4.5. It can be observed in the Fig. 4.6 that increasing particle stiffness shifts the attenuation curves upward without changing the tharacteristic shape of the curve. This produces less total attenuation, but with a similar re attenuation rate.

\subsubsection{Effects of Viscosity, Gap Distance and Particle Stiffness on Wave Speed}

Fig. 4.7 shows the effects of gap distance and viscosity on the wave speed. As expected, the wave speed decreases with increase in the gap distance, and when the gap is small, these effects are more pronounced. As with the effect on wave attenuation, viscosity does not have much influence on wave speed. An increase of fluid viscosity leads to a small increase in the wave speed, and again this variation is more apparent at small gap distances. These findings are supported by experimental results, eg. Nur and Simmons (1969), and by particular theoretical models, Dvorkin et al. (1994). As expected, wave speed is found to be higher through assemblies of stiffer particles (see Fig. 4.8).

\subsubsection{Effect of Input Pulse Duration on Wave Attenuation}

This section presents the results of an investigation on the effects of wavelength (or equivalently, input pulse duration) on wave propagation in saturated granular materials. The effect of wavelength on dynamic load transfer in dry granular media has been experimentally investigated by Xu (1991) and Shukla et al. (1993). In his experiments, Xu studied wave motion in one-dimensional granular materials simulated by

a single chain of circular disks of Homalite material. For a given particle diameter, long input wavelengths produce a wave pulse which decomposed into a series of propagating oscillatory signals (ringing phenomenon) resulting fron microstructural nonlinearities. It was found that this ringing phenomenon occured only with long wävelengths. As a result 
of this behavior, wave amplitude did not show the usual monotonic decrease with the Mopagational distance.

The numerical results from the DEM model for a single straight chain are shown in Figs. 4.9 and 4.10. Fig. 4.9 shows the contact load histories at three different points along the simulated granular disk chain for a short period loading of $5 \mu \mathrm{s}$. The wave amplitude does not show any monotonic decrease with the propagation distance, and the wave dispersion is small. This happens due to small wavelength to disk diameter ratio. The dynamic responses of the granular assembly to long period loadings show significant differences from the result for short input loading. Fig. 4.10 illustrătes three contact load profiles at different contact points for the case of a $10 \mu \mathrm{s}$ input loading period. A ringing phenomenon can be observed in the three profiles shown. The amplitudes of the ringing oscillations decreases with propogational distance whereas the time intervals between the oscillations increases. Moreover, in contrast to the short input loading case, the peak amplitudes are not always smaller than the value of the input loading amplitude for long wavelength case. Instead of decreasing monotonically the peak contact loads first increase with propagational distance, and then they will begin to decrease steadily. It appears that for high input wave length to disk diameter ratio, the propagating signal produces oscillatory motion within influenced group of particles, which leads to ringing behavior. This redistribution of energy produces a wave profile with a peak amplitude higher than the input at particular contact points. Earlier,Tai (1994) had numerically investigated the effects of wavelength on wave propagation in a one-dimensional dry granular medium simulated by a single chain of circular disks, and had found similar effects. Fig. 4.11 shows the effect of input pulse duration on wave propagation for dry case. It is clear from the Fig. 4.11 that in dry case also, ringing phenomenon is observed at high input wave length, and the peak amplitudes are not always smaller than the input loading amplitude. 


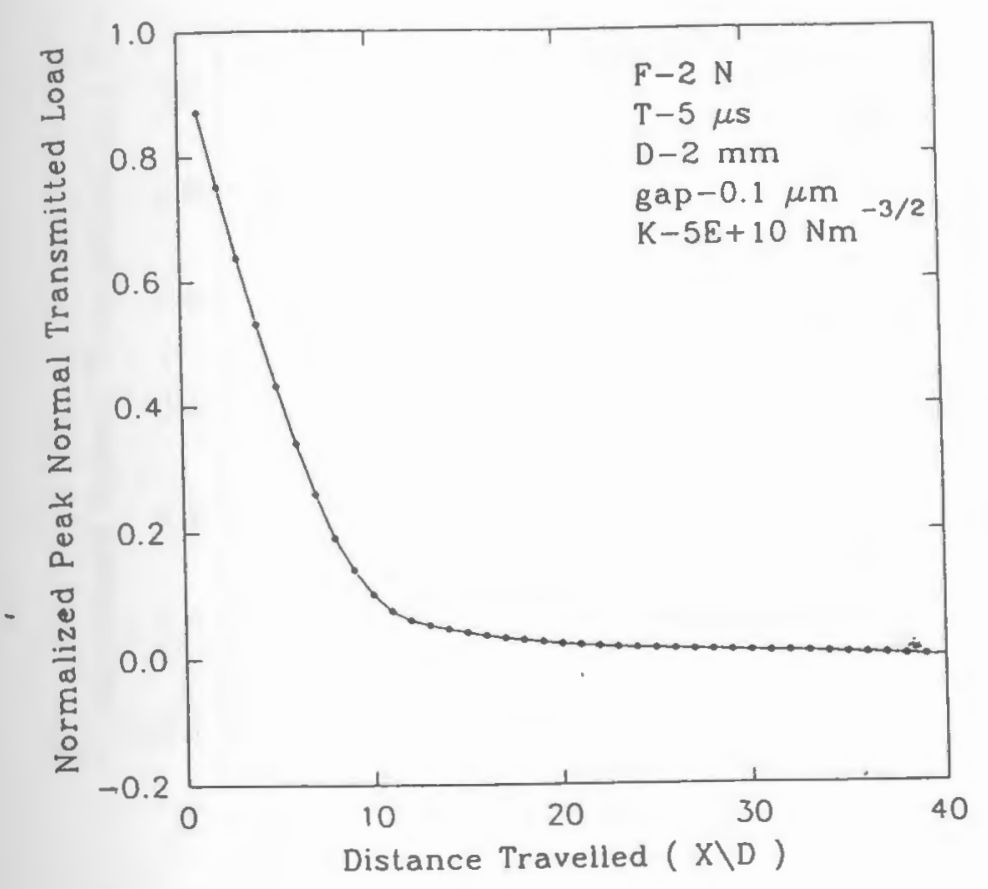

Fig. 4.2 Peak normal transmitted load vs. distance in a single straight chain

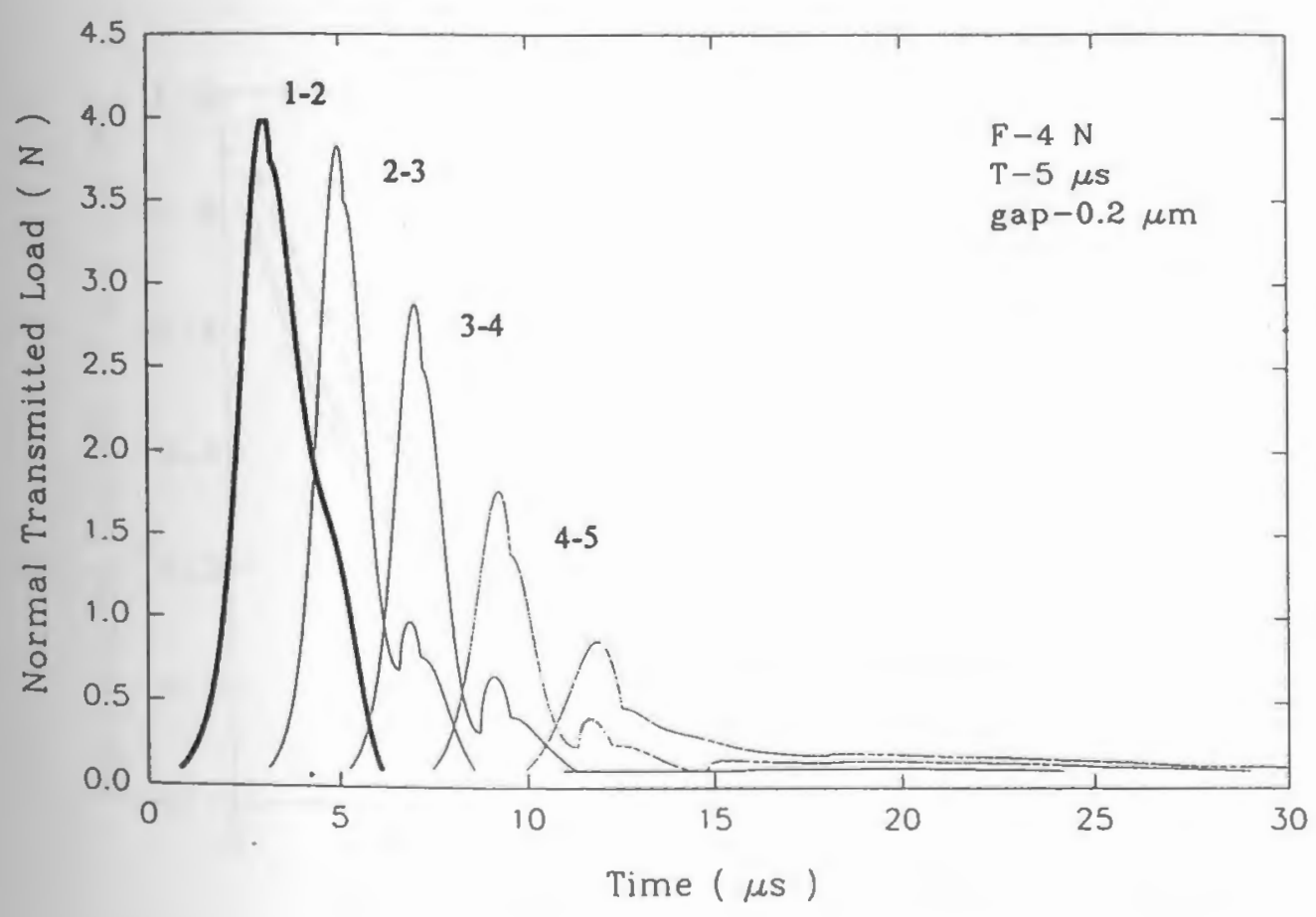

Fig. 4.3 Normal transmitted load vs. time in a single straight chain 


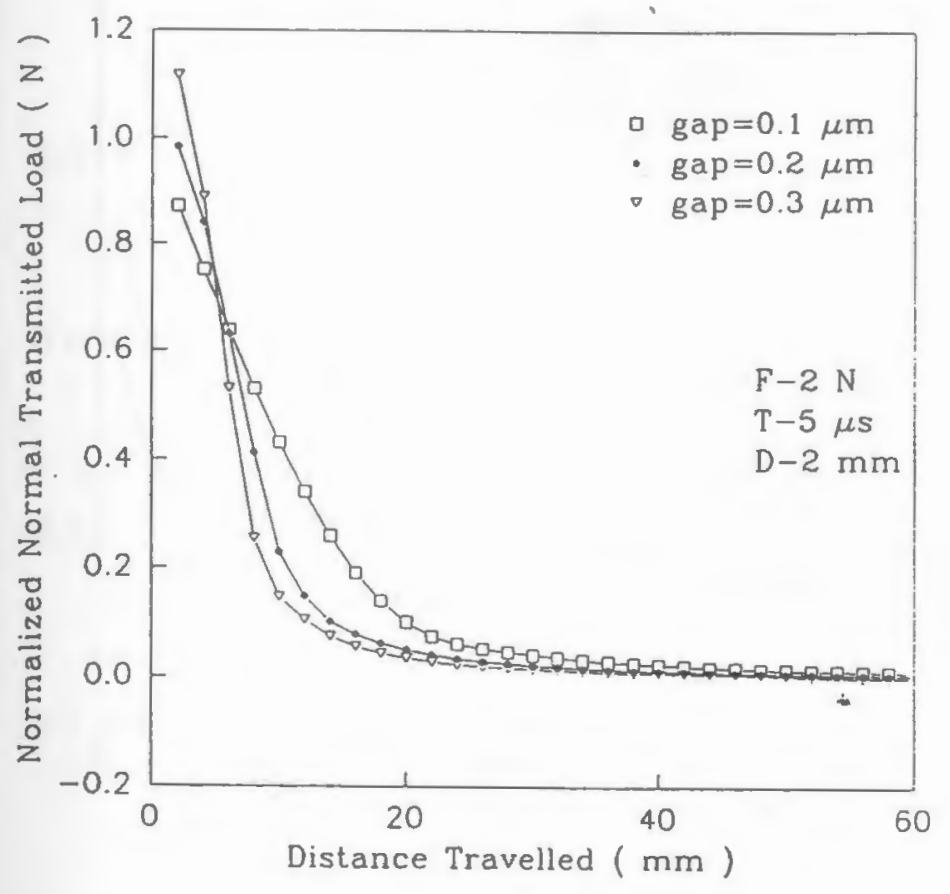

Fig. 4.4 Normalized transmitted load vs. distance for different interparticle gap spacing

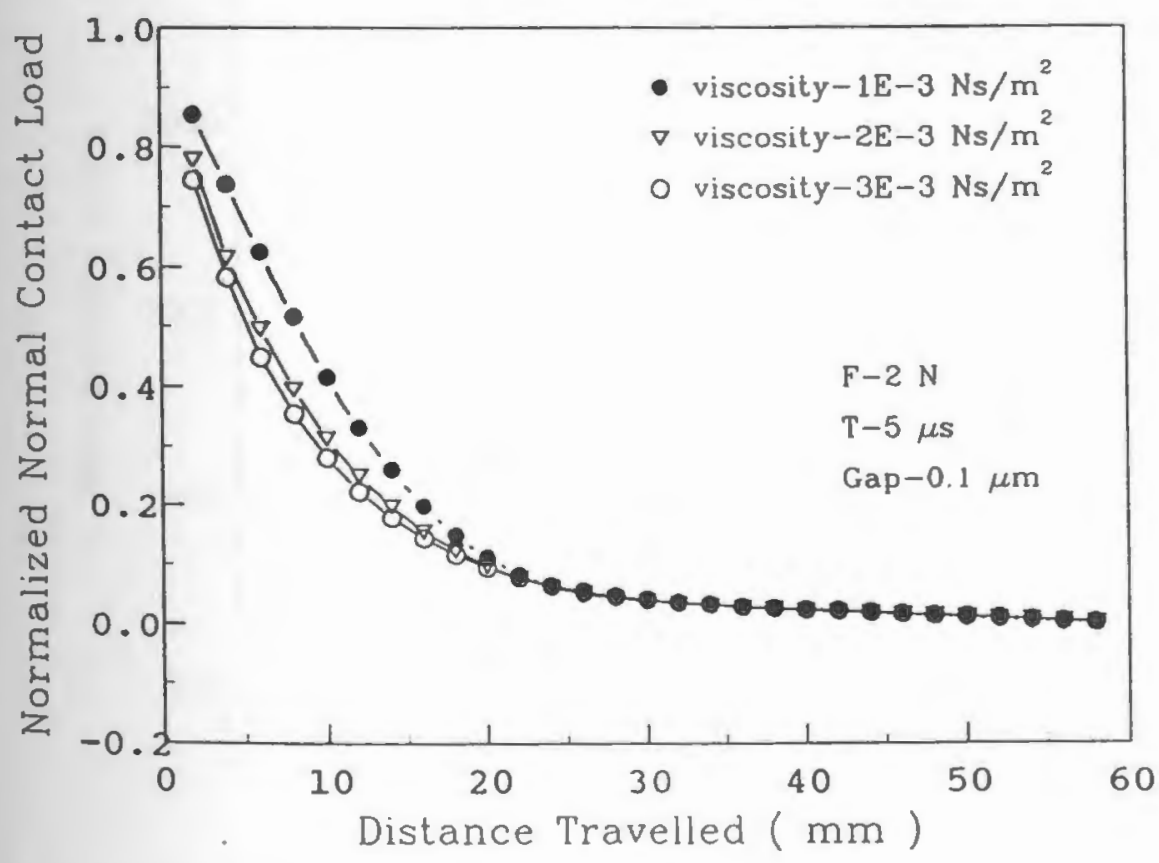

Fig. 4.5 Normalized transmitted load vs. distance for various pore fluid viscosity 


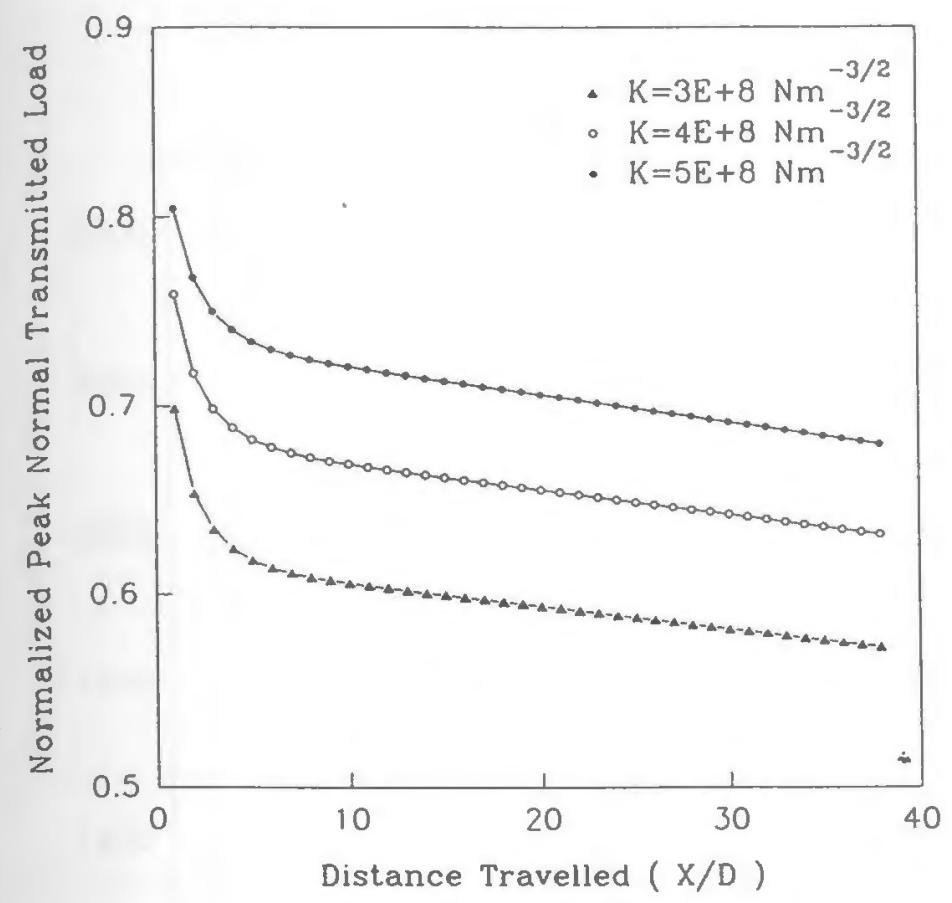

Fig. 4.6 Normalized peak contact load vs. distance for different particle stiffnesses

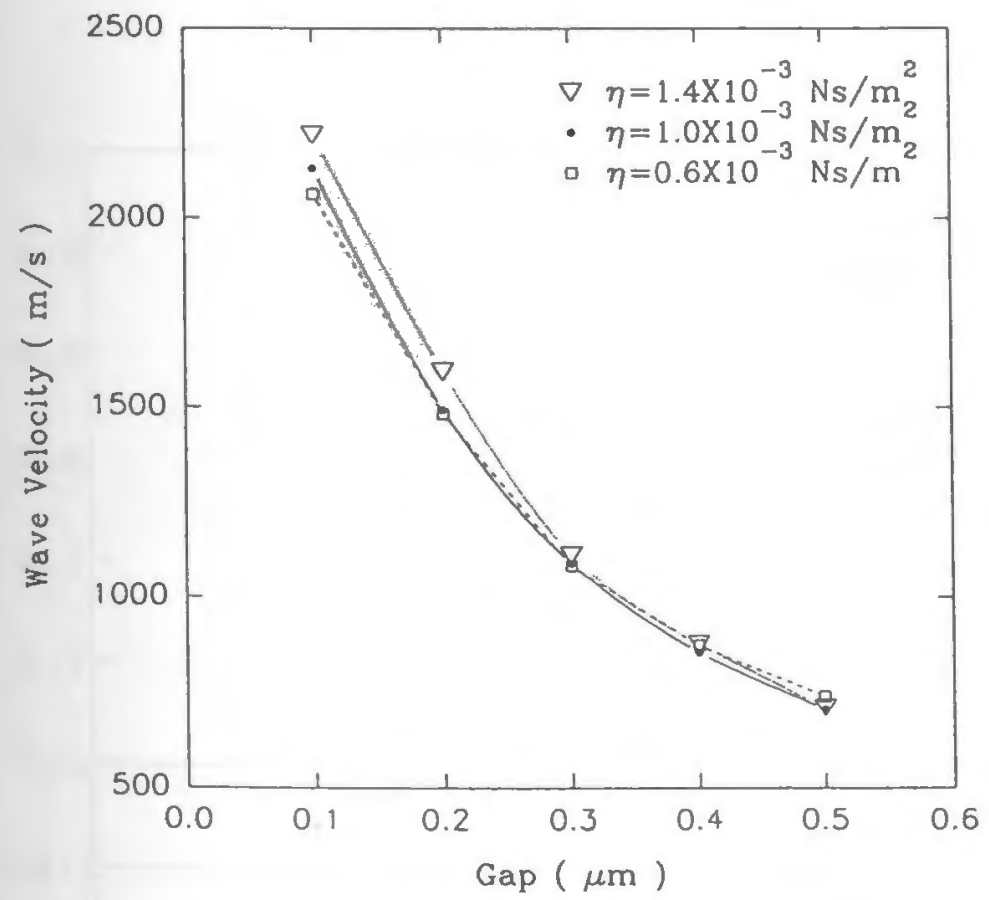

Fig. 4.7 Plot of wave velocity vs. interparticle gap spacing at different viscosities 


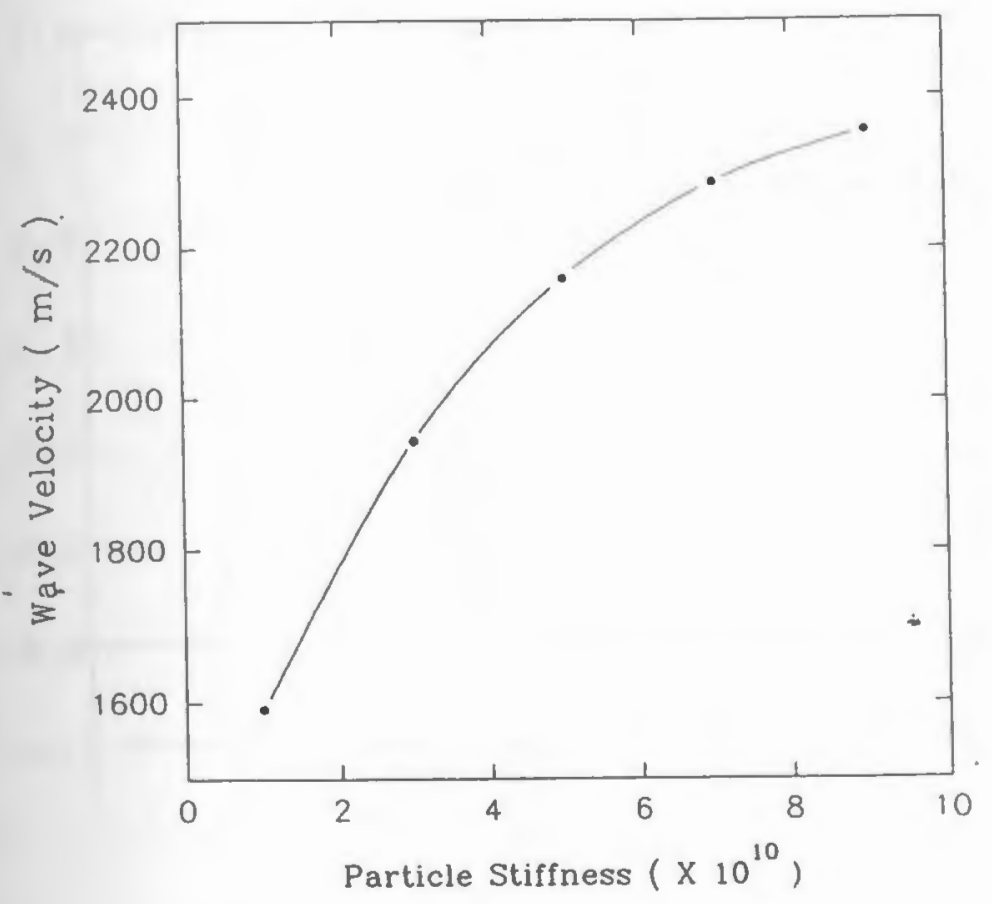

Fig. 4.8 Plot of wave velocity vs. particle stiffness

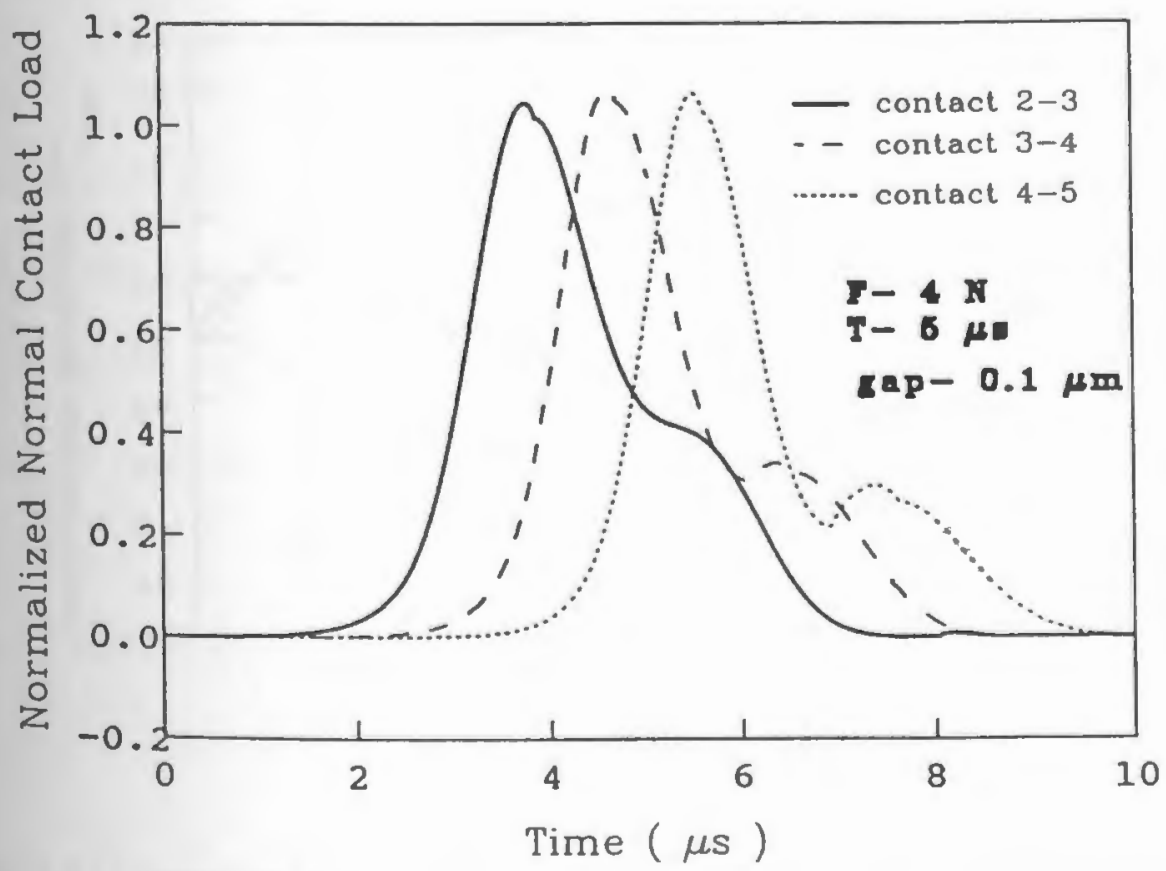

Fig. 4.9 Contact load history at three contact points along the single chain for $\mathrm{T}=5 \mu \mathrm{S}$ 


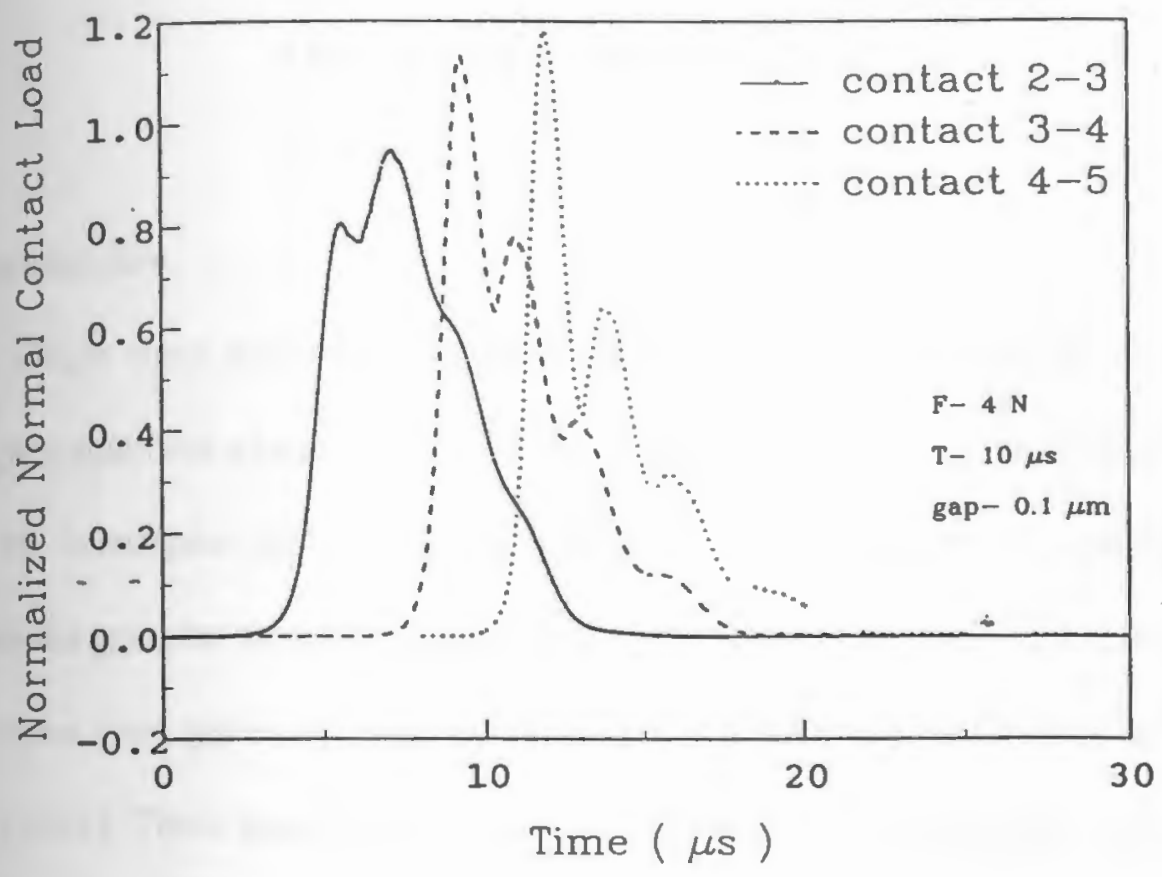

Fig. 4.10 Contact load history at three contact points along the single chain for $\mathrm{T}=10 \mu \mathrm{s}$

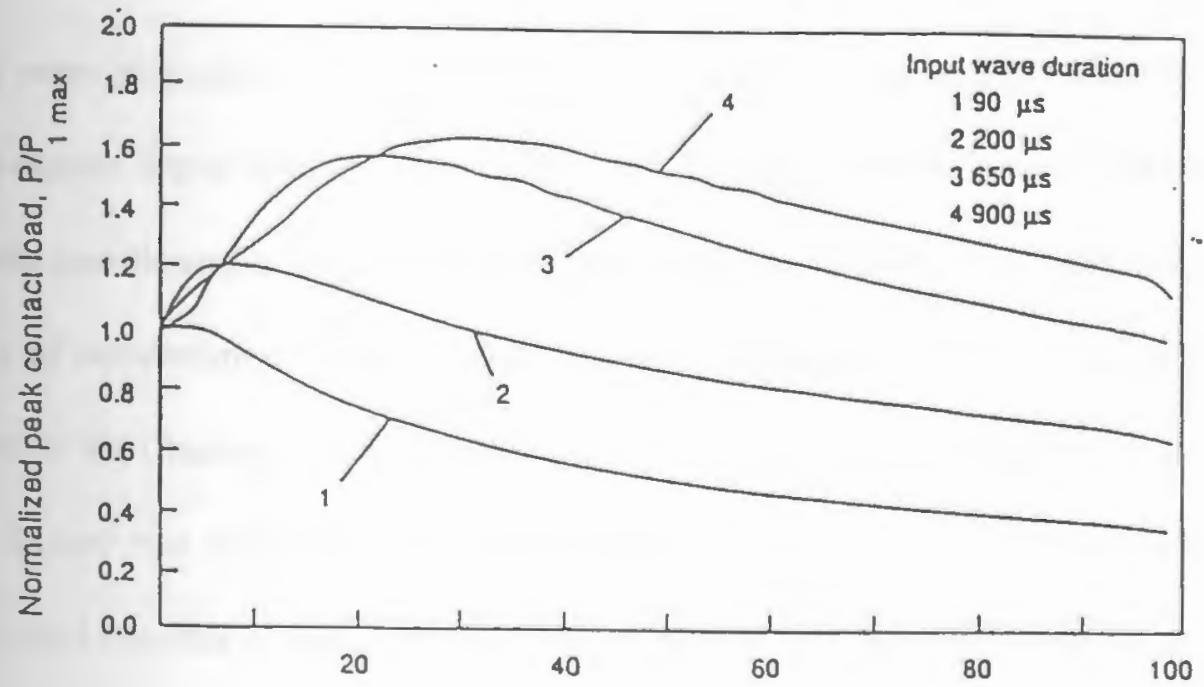

Normalized propagational distance, XD

Fig. 4.11 Normalized peak contact loads vs propagational distance for different input wave durations (dry case) 


\section{Chapter 5}

\section{TWO-DIMENSIONAL STUDIES}

\subsection{Introduction}

Single chain simulations as discussed in the Chapter 4 do not ascertain the true wave propagational characteristics as real granular materials are multi-dimensional. In order to investigate the use of the model for multi-dimensional simulations, twodimensional granular model assemblies were generated. Random assỉemblies with different anisotropies were generated using the computational random media generators developed by Tai (1991). These generating codes can create large random particulate assemblies with varying degrees of microstructural fabric such as porosity, coordination number, particle contact normal and branch vector distributions, void vectors, etc.

Fig. 5.1 shows an example assembly created using one of the generators. Dynamic input for wave propagation was created through loading of particles along left and bottom edges as shown. Input loadings were applied with identical magnitude and time history as used in the one-dimensional simulations. Values of the model parameters were assumed on the basis of simulations of wave propagation through single chains of circular disks as discussed in the Chapter 4 . The transmitted wave output (measured by the inter-particle contact forces) was collected among the particles along the right and top sides of the assembly, and thus this technique can provide comparisons of horizontal and vertical wave propagational behaviors including wave speed and amplitude attenuation. 
The next section briefly describes the generation of random granular assemblies with various anisotropies and microstructures.

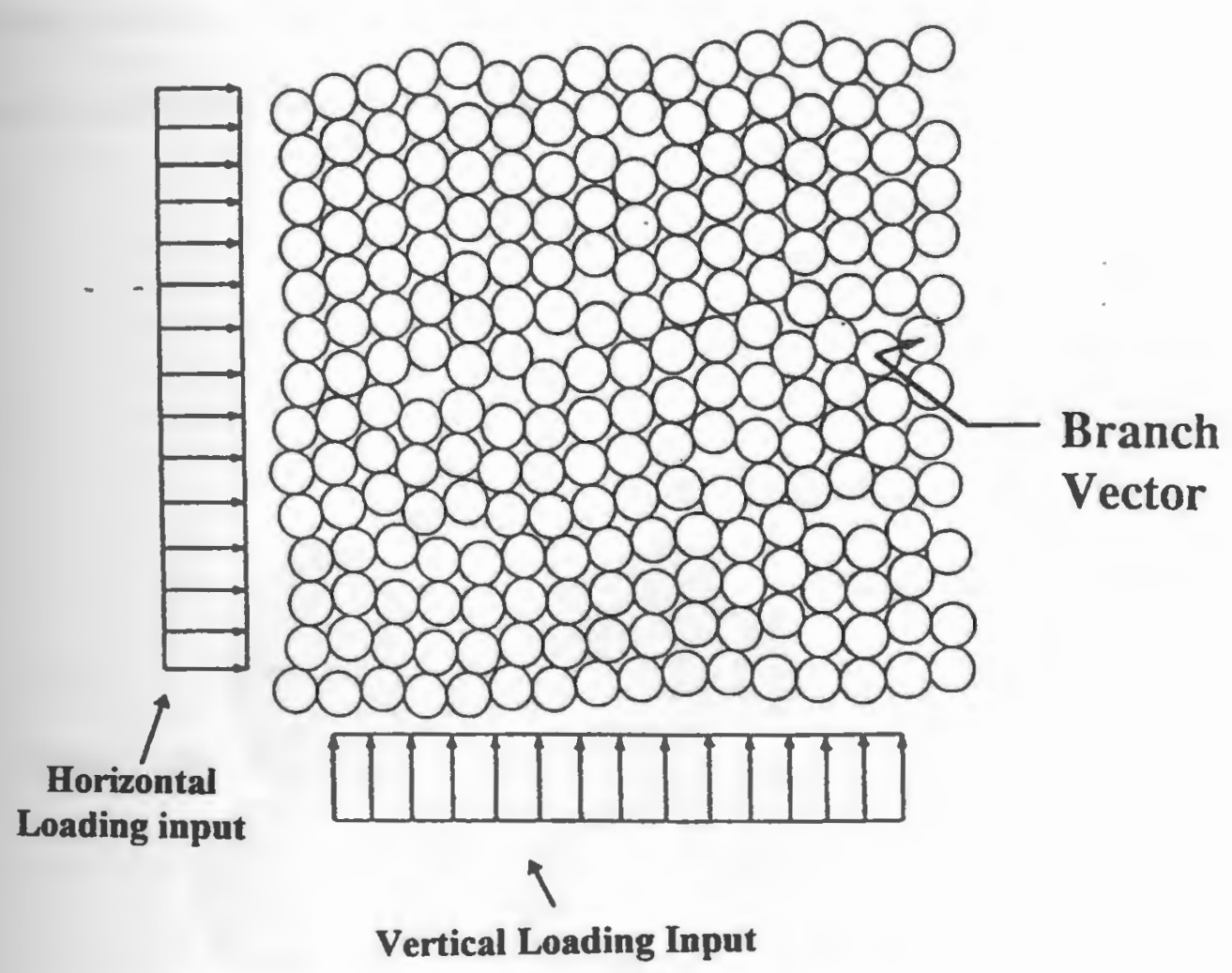

Fig. 5.1 A random two-dimensional assembly

\subsection{Assembly generators}

Random assembly generating schemes, which can create assemblies with different microstructures or fabric, are required in order to study the effects of granular anisotropy and microstructure on wave motion in particulate materials. The distribution of local 
branch vectors $\mathbf{b}$ which connect mass centers of neighboring particles in contact is one of several measures of anisotropy that have been used in particulate materials (see Fig. 5.2). It is this measure which was used by Tai (1994) to develop various assembly generating schemes. Powell (1980), Visscher (1972), and Bagster (1985) designed different assembly generating schemes using other anisotropy measures such as coordination number, contact normal vector, porosity etc. (Fig. 5.2).

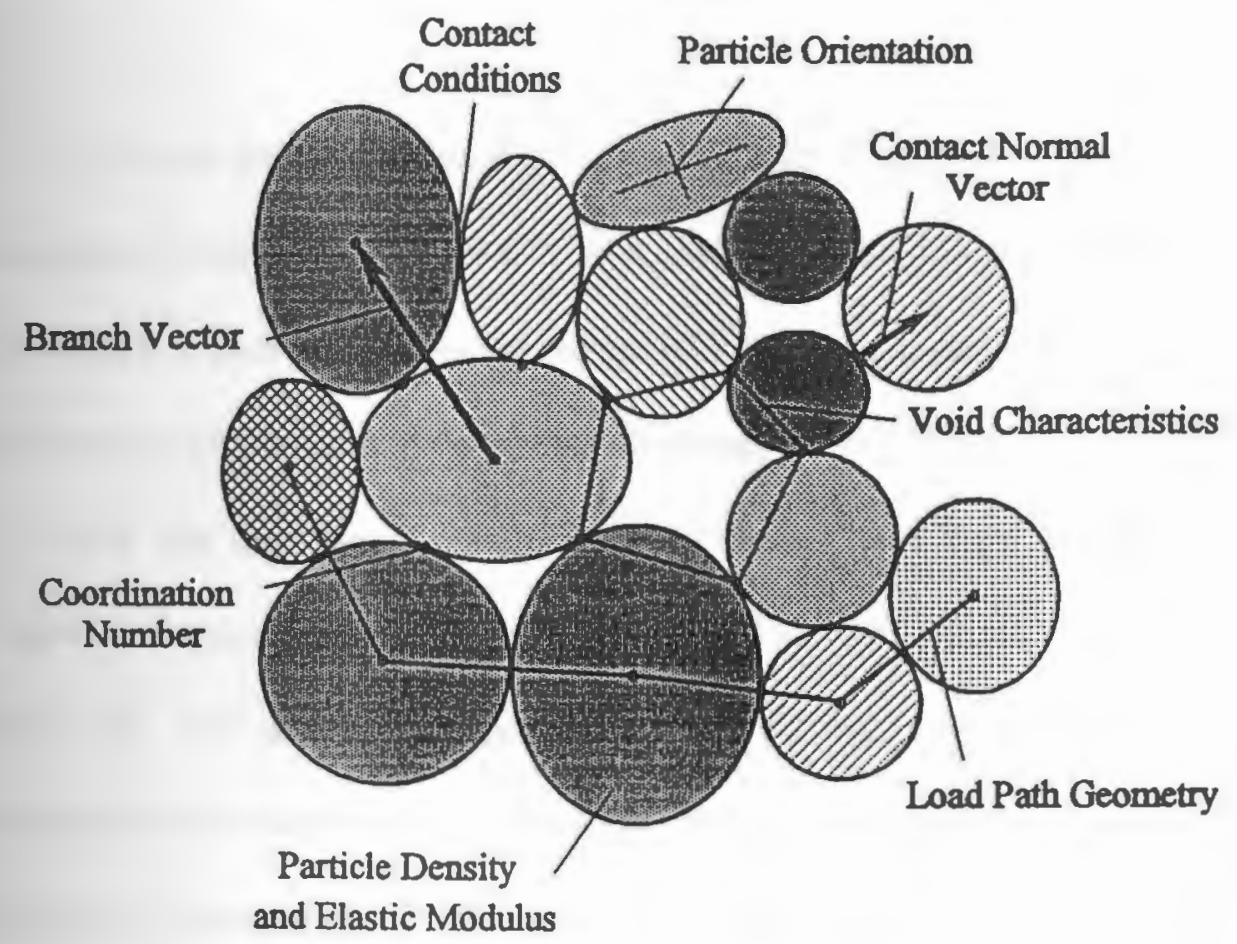

Fig. 5.2 Various fabric measures used for particulate materials 


\subsubsection{Strongly Anisotropic Generator}

This generating code creates assemblies with very high degrees of anisotropy in two orthogonal directions. The scheme employed to generate such types of assemblies can be explained in the following steps.

1. First, a base horizontal layer of circular disks with spacings between neighboring disks centers varying randomly from one to two disk diameters is created (see Fig. 5.3a).

2. A second layer of disks is generated by using each pair of neighboring disks in the base layer and generating a uniquely positioned disk in contact with the pair (see Fig. $5.3 b)$.

3. The next layer is generated using pairs of neighboring disks in the previous layer and randomly forming disks in contact with them with the constraint that the angle of branch vectors of each newly constructed contact pair lie in a particular range $\left( \pm 15^{\circ}\right.$ for the case shown) with respect to the vertical direction, see Fig. 5.3c.

4. For the next layer, the positions of the left and right boundary disks are determined in a special manner. The left boundary disk is constrained to lie in the branch angle of $115^{\circ}-135^{\circ}$ measured counter-clockwise from the horizontal in contact with the left most disk of the previous layer. Similarly, the right boundary disk is generated with a random branch vector angle in the range of $45^{\circ}-75^{\circ}$ (see Fig. 5.3d). Remaining disks are created by following step 2 above as shown in the Fig. 5.3e.

5. Remaining layers are generated by following steps $3 \& 4$ until the assembly has the required number of disks. 
At any stage if there is overlap between the disks, the newly generated disk is replaced with a new trial disk, and in this way large random strongly anisotropic assemblies can be created without overlap between the disks. Fig. 5.6 shows an assembly generated using this scheme, and Fig. 5.7 illustrates a polar plot of the local branch vector orientation distribution of this assembly.

\section{0}

(a)

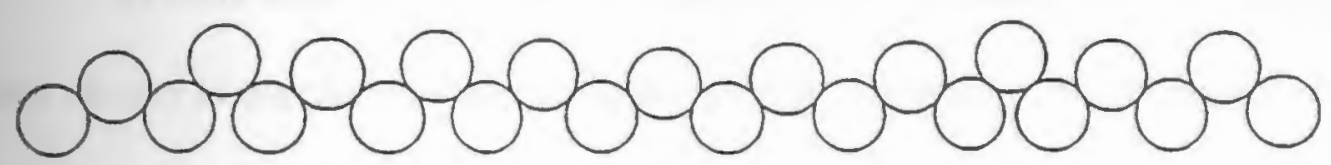

(b)

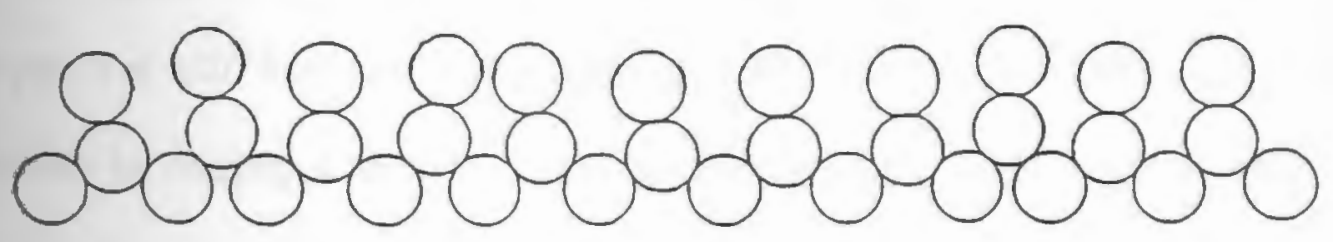

(c)

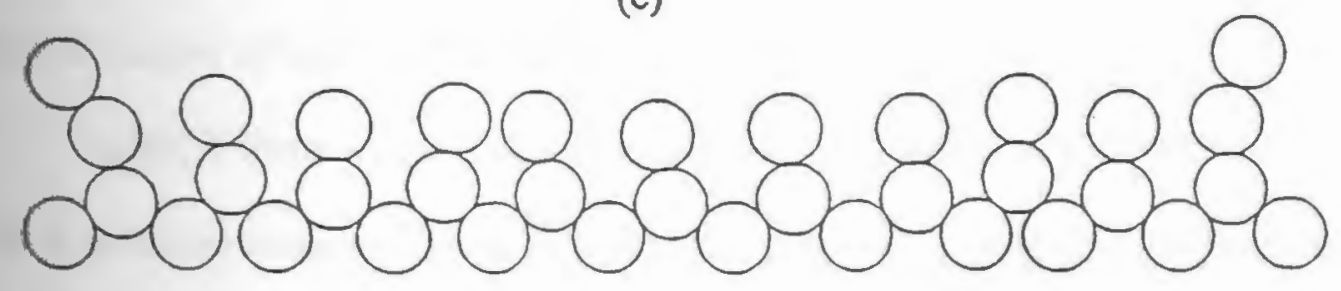

(d) 


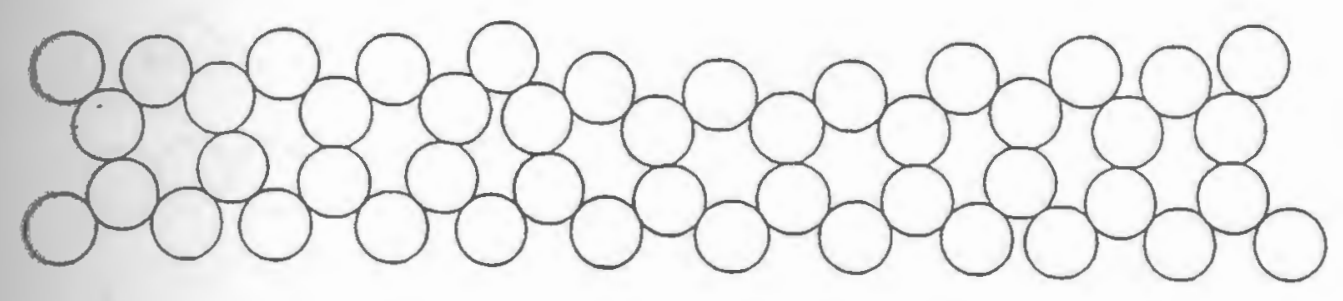

(e)

Fig. 5.3 Strongly anisotropic media generator (Tai, 1994)

\subsubsection{Moderately Anisotropic Generator}

The second type of assembly generator was developed for creating assemblies with moderate anisotropy. The algorithms employed can be described in the following steps.

1. An initial base layer of contacting disks is formed in such a way that the angle of branch vectors of any pair of neighboring disks fall in the range $\alpha= \pm 20^{\circ}$ measured from the horizontal (see Fig. 5.4a).

2. The next layer is generated with the left disk of the new layer in contact with the left most disk of the previous layer and randomly positioned such that the branch vector of the pair is at $\pm 20^{\circ}$ from the vertical (see Fig. 5.4b). The rest of the disks are generated randomly by creating disks in contact either with their left neighbor and the disk in the previous layer or with their left neighbor only. This procedure is repeated until the required number of layers are generated.

Again, if there is overlap between the disks at any stage of development, a new disk is generated to replace it. Fig. 5.8 shows an assembly created with this generator and Fig. 5.9 illustrates the branch vector distribution as a function of the angular direction. 


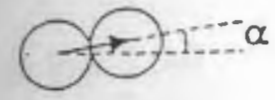
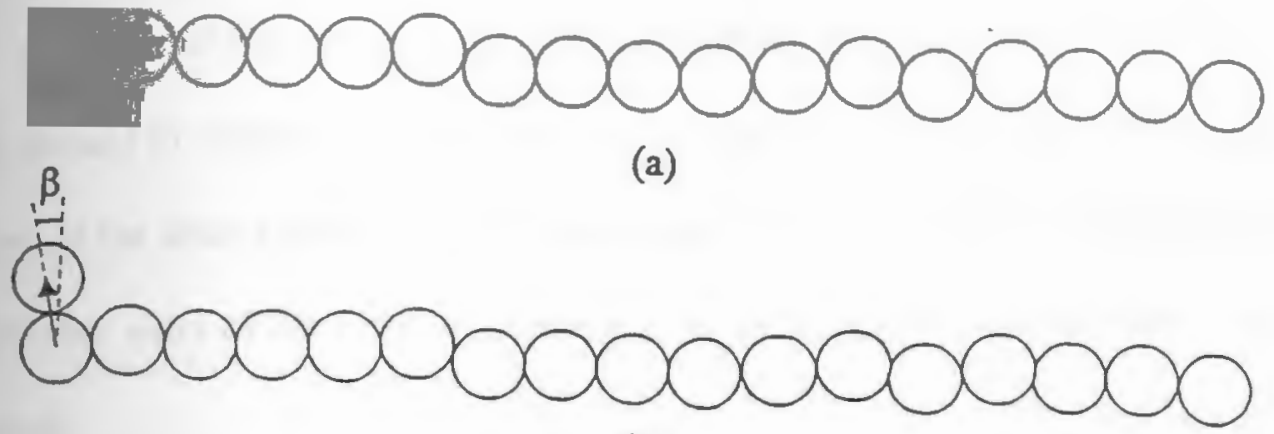

(b)

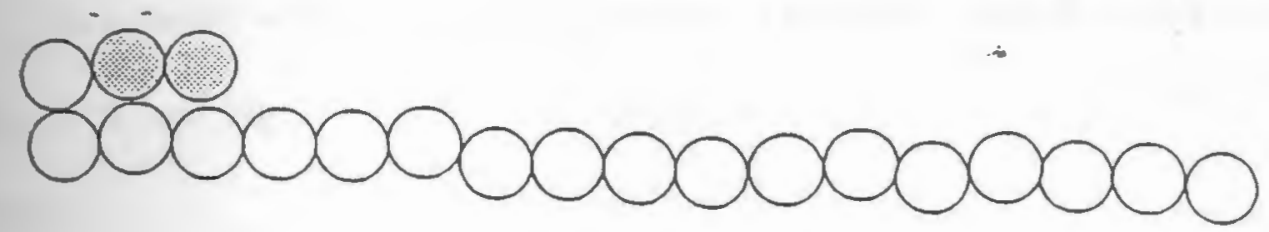

(c)

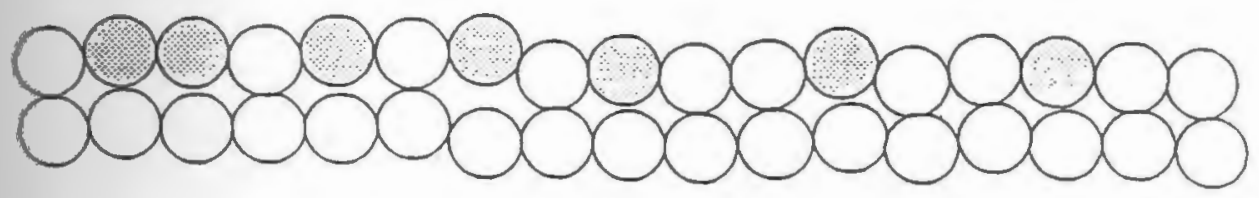

(d)

Fig. 5.4 Moderately anisotropic generator (Tai, 1994)

\subsubsection{Weakly Anisotropic Generator}

The steps employed for generating weakly anisotropic assemblies can be enumerated as follows.

1. A disk forming the central point of the assembly is created. From this initial disk a branch angle is randomly generated to determine the position of a new contacting disk. 
This procedure is used to randomly generate the first five disks in contact with the central disk (see Fig. 5.5a).

2. A pair of disks is randomly selected from the existing assembly and then a new disk is created in contact with these two disks. This new disk is discarded if it overlaps with any of the disks already in the existing assembly. This procecture is repeated over all possible disk pairs of the existing assembly until the desired number of disks have been generated.

An assembly with small degree of anisotropy generated using this scheme is shown in Fig. 5.10, and Fig. 5.11 is the polar distribution plot of the branch vectors for this assembly.
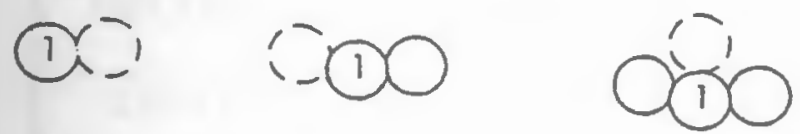

(a)
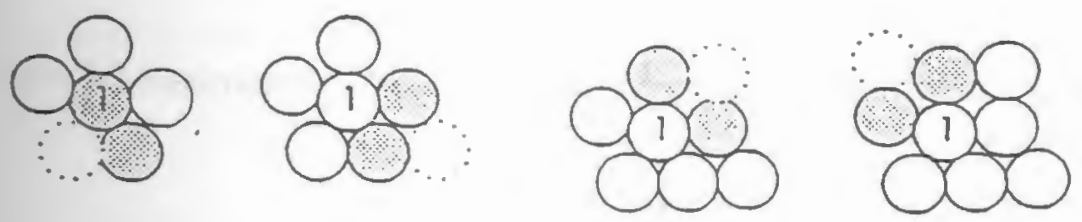

(b)
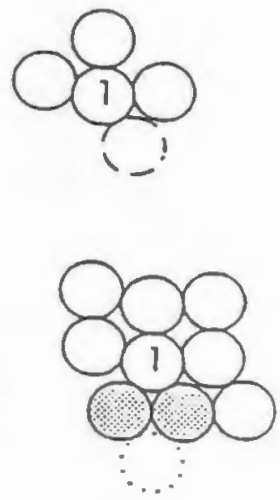

Fig. 5.5 Weakly anisotropic generator (Tai, 1994) 


\subsection{Two-Dimensional Simulations Results}

This section presents the simulation results of two-dimensional wave propagation and dynamic load transfer through saturated particulate media using the discrete element computational code developed. Several two-dimensional assemblies with varying degree of fabric anisotropy were created with the various random media generators, discussed previously. The typical values of computational model parameters used in the code for these simulations are given in Table 5.1.

\begin{tabular}{||c|c|}
\hline PARAMETER & VALUE \\
\hline NT (no. of time-increments) & 40,000 \\
\hline DT (time-increments) & $5 \times 10^{-9} \mathrm{~s}$ \\
\hline CC (particle stiffness) & $4 \times 10^{10} \mathrm{Nm}^{-3 / 2}$ \\
\hline HH (influence zone width) & $2 \times 10^{-7} \mathrm{~m}$ \\
\hline DELTA (calculation parameter) & 0.5 \\
\hline BATA (calculation parameter) & 0.25 \\
\hline
\end{tabular}

Table 5.1 Parameters used in the numerical analysis of two-dimensional assemblies

DELTA and BATA are the interpolation parameters, which are used in the numerical integration scheme to determine the current velocity at any time-step from the current acceleration and velocity at the previous time-step. 
Sub-sections 5.3.1, 5.3.2 and 5.3.3 present the wave propagational behavior through strongly anisotropic, moderately anisotropic and weakly anisotropic assemblies respectively.

\subsubsection{Simulations Results for Strongly Anisotropic Assembly}

An assembly with a high degree of anisotropy was generated using the strongly anisotropic random media generator (see Fig. 5.12). The assembly has 378 circular particles, and is assumed to be saturated with water (viscosity $=0.001 \mathrm{Ns} \mathrm{m}^{-2}$. Porosity, defined as the ratio of void volume to total volume of the sample, of this assembly was calculated to be $31.70 \%$. In order to correlate material microstructure to the wave propagational behaviors, the branch vector distribution of the assembly in Fig. 5.12 was computed. As mentioned previously, the branch vector is a commonly used microstructural fabric measure that relates the relative positions between particles in contact. Fig. 5.13 illustrates a polar diagram plot of the branch vector distribution for the assembly shown in the Fig. 5.12. It can be observed from the distribution plot that this model material system shows preferred directions (anisotropy) with more branch vectors distributed in the vertical direction than in the horizontal. Thus we would expect more wave transmission for the vertical loading case as compared to that for horizontal case.

Discrete element simulations were run for the assembly shown in Fig. 5.12. Fig. 5.14 illustrates the normalized average wave transmission through the assembly for the horizontal and vertical loading cases. The interparticle gap in this case is $0.1 \mu \mathrm{m}$. The vertical scale represents the average of all interparticle load transfers at the output sides 
normalized with respect to the sum of input loadings (see Fig. 5.1). These results show that much less wave attenuation occurs in the vertical direction than in the horizontal. This indicates that the wave transmission is clearly related to the anisotropic fabric of the model particulate material (see Fig. 5.13). The wave velocities in the vertical and horizontal directions are $1105 \mathrm{~m} / \mathrm{s}$ and $780 \mathrm{~m} / \mathrm{s}$ respectively, again indicating a correlation with the model's fabric. Fig. 5.15 shows vertical propagation results of the same model material for the case with different interparticle gap spacing. It can be observed that with an increase of the gap spacing, attenuation increases while propagational speed decreases. For the case of vertical propagation, the wave speed is $1150 \mathrm{~m} / \mathrm{s}$ for $0.1 \mu \mathrm{m}$ spacing, and $538 \mathrm{~m} / \mathrm{s}$ for $0.2 \mu \mathrm{m}$ gap spacing. Fig. 5.15 was redrawn with transmitted load normalized with respect to contact load in the vertical direction at the inside first layer from the bottom of the assembly, and the results are shown in the Fig. 5.16. It can be seen that transmitted load normalized with respect to the contact load at the first layer in the vertical direction is 1.28 and 1.44 times the transmitted load normalized with respect to the input load for 0.1 and $0.2 \mu \mathrm{m}$ interparticle gap spacings respectively. These results are consistent with the one-dimensional single chain simulations.

Wavelengths effects are also numerically studied for the two-dimensional case. A vertical plane wave loading is simulated by simultaneous application of a loading to each bottom disk of the assembly (see Fig. 5.1). Each input loading is a triangular time dependent pulse with a peak value of $2 \mathrm{~N}$ and durations were varied including values of 5 $\mu \mathrm{s}, 10 \mu \mathrm{s}$ and $50 \mu \mathrm{s}$. The interparticle gap spacing was held fixed at $0.1 \mu \mathrm{m}$. The average contact forces at the output (top) side normalized with respect to the input loadings are 
shown in the Figs. 5.17 and 5.18 as a function of time for different pulse durations. Consistent with the one-dimensional case and earlier observations by Shukla et al. (1993) for dry materials, the ringing phenomenon tendency is also observed in the twodimensional assembly for longer input pulse duration ( $50 \mu \mathrm{s})$. No ringing phenomenon occurs in the cases of short durations of $5 \mu \mathrm{s}$ and $10 \mu \mathrm{s}$. The propagational speed is 1105 $\mathrm{m} / \mathrm{s}, 1448 \mathrm{~m} / \mathrm{s}$ and $1272 \mathrm{~m} / \mathrm{s}$ for the $5 \mu \mathrm{s}, 10 \mu \mathrm{s}$ and $50 \mu \mathrm{s}$ inputs respectively.

To investigate the particle size effects on wave propagation through twodimensional model assemblies, an identical strongly anisotropic assembly (shown in the Fig. 5.19) was created with the same branch vectors distribution or fabric as that shown in the Fig. 5.12, but with a different particle diameter of $1 \mathrm{~mm}$. The input load period and amplitude were kept constant. Fig. 5.20 shows the comparison of vertical transmission through the assembly for different particle diameters. The wave speed for the $1 \mathrm{~mm}$ particle case was found to be higher, and so was the transmitted load per meter of distance propagated (see Table 5.2). The smaller particle case was run again with a different peak value of $1 \mathrm{~N}$ to keep the input stress the same as that used for the $2 \mathrm{~mm}$ particle diameters case. The results are shown in the Fig. 5.21, and again, it is seen that more load is transmitted per meter of distance propagated.

Table 5.2 summarizes the strongly anisotropic assembly simulations results. 


\begin{tabular}{|c|c|c|c|c|c|c|}
\hline $\begin{array}{c}\text { Radius } \\
\mathbf{R}(\mathbf{m m})\end{array}$ & $\begin{array}{c}\text { Gap } \\
\mathbf{g} \\
(\boldsymbol{\mu} \mathbf{m})\end{array}$ & $\begin{array}{c}\text { Peak } \\
\text { Load } \\
\mathbf{P}(\mathbf{N})\end{array}$ & $\begin{array}{c}\text { Time } \\
\text { Period } \\
\mathbf{T}(\boldsymbol{\mu s})\end{array}$ & $\begin{array}{c}\text { Propagational } \\
\text { Direction }\end{array}$ & $\begin{array}{c}\text { Wave } \\
\text { Velocity } \\
\mathbf{V}(\mathbf{m} / \mathbf{s})\end{array}$ & $\begin{array}{c}\text { Normalized } \\
\text { Transmitted Load } \\
\text { per } \mathbf{~ m ~}(\% / \mathbf{m})\end{array}$ \\
\hline 1 & 0.1 & 2 & 5 & vertical & 1105 & 1.190 \\
\hline 1 & 0.2 & 2 & 5 & vertical & 538 & 0.607 \\
\hline 1 & 0.1 & 2 & 5 & horizontal & 1024 & 0.048 \\
\hline 1 & 0.1 & 2 & 10 & vertical & 1448 & 5.833 \\
\hline 1 & 0.1 & 2 & 50 & vertical & 1272 & 64.285 \\
\hline 0.5 & 0.1 & 2 & 5 & vertical & 1235 & 12.857 \\
\hline 0.5 & 0.1 & 1 & 5 & vertical & 955 & 5.142 \\
\hline
\end{tabular}

Table 5.2 Simulation results for strongly anisotropic assembly

\subsubsection{Simulations Results for Moderately Anisotropic Assembly}

Fig. 5.22 shows a moderately anisotropic assembly with 441 particles generated by using the moderately anisotropic random media generator, and the branch vector fabric of this assembly is illustrated in Fig. 5.23. It can be observed from the branch fabric distribution that this assembly has a slight preference for wave transmission in the horizontal direction. Porosity for this assembly was $21.46 \%$. Results of the horizontal and vertical wave motions through this assembly for different interparticle spacings are shown in Figs. 5.24, 5.25 and 5.26. The computational model parameters used in this case are identical to those of the previous example.

As expected, wave attenuation is less in the horizontal direction, thus correlating with branch fabric (see Fig. 5.24). But the difference in the transmitted load for the two directions is much less than observed in the strongly anisotropic assembly. Also, it can be 
observed from Figs. 5.25 and 5.26 that wave transmission and wave speed are highly dependent on the spacings between particles, with dependency similar to the previous highly anisotropic case.

The effect of particle size on wave transmission was also studied for the moderately anisotropic assembly. From Figs. 5.27, 5.28 and 5.29, it is clear that particle size has the effect as was observed for strongly anisotropic case, i.e. as the particle size is reduced, wave speed increases, but wave attenuation decreases.

The simulation runs for the moderately anisotropic assembly are summarized in Table 5.3.

\begin{tabular}{|c|c|c|c|c|c|c|}
\hline $\begin{array}{c}\text { Radius } \\
\mathbf{R}(\mathbf{m m})\end{array}$ & $\begin{array}{c}\text { Gap } \\
\mathbf{g} \\
(\mu \mathrm{m})\end{array}$ & $\begin{array}{c}\text { Peak } \\
\text { Load } \\
\mathbf{P}(\mathbf{N})\end{array}$ & $\begin{array}{c}\text { Time } \\
\text { Period } \\
\mathbf{T}(\mu \mathbf{s})\end{array}$ & $\begin{array}{c}\text { Propagational } \\
\text { Direction }\end{array}$ & $\begin{array}{c}\text { Wave } \\
\text { Velocity V } \\
(\mathbf{m} / \mathbf{s})\end{array}$ & $\begin{array}{c}\text { Normalized } \\
\text { Transmitted Load } \\
\mathbf{p e r} \mathbf{m}(\% / \mathbf{m})\end{array}$ \\
\hline 1 & 0.1 & 2 & 5 & vertical & 1206 & 0.065 \\
\hline 1 & 0.2 & 2 & 5 & vertical & 528 & 0.023 \\
\hline 1 & 0.1 & 2 & 5 & horizontal & 1169 & 0.447 \\
\hline 1 & 0.2 & 2 & 5 & horizontal & 527 & 0.197 \\
\hline 0.5 & 0.1 & 2 & 5 & vertical & 1357 & 2.105 \\
\hline 0.5 & 0.1 & 2 & 5 & horizontal & 1583 & 2.632 \\
\hline
\end{tabular}

Table 5.3 Simulation results for the moderately anisotropic assembly 


\subsubsection{Simulations Results for Weakly Anisotropic Assembly}

A weakly anisotropic assembly and its branch vectors distribution are shown in Figs. 5.30 and 5.31. This assembly has slightly more branch vectors in the vertical direction, so we would expect a little more attenuation for horizontal propagation when compared with vertical transmission. The number of particles in the assembly is 179 and porosity is $22.36 \%$. Again, computer simulations were run for horizontal and vertical wave propagation for gap spacings of $0.1 \mu \mathrm{m}$ and $0.2 \mu \mathrm{m}$, and results are shown in the Figs. 5.32, 5.33, 5.34 and 5.35 .

From Figs. 5.32 and 5.33, it is clear that the wave attenuation is little higher in the horizontal propagation as compared to the vertical case. Also, we see that with increasing interparticle gap spacings, the attenuation increases and the propagational speed decreases (see Figs. 5.34 and 5.35).

The summary of simulation runs for the weakly anisotropic assembly is given in Table. 5.4.

\begin{tabular}{|c|c|c|c|c|c|c|}
\hline $\begin{array}{c}\text { Radius } \\
\mathbf{R} \\
(\mathbf{m m})\end{array}$ & $\begin{array}{c}\text { gap } \\
\mathbf{g} \\
(\mu \mathrm{m})\end{array}$ & $\begin{array}{c}\text { Peak } \\
\text { Load } \\
\mathbf{P}(\mathbf{N})\end{array}$ & $\begin{array}{c}\text { Time- } \\
\text { Period } \\
\mathbf{T}(\mu \mathbf{s})\end{array}$ & $\begin{array}{c}\text { Propagational } \\
\text { Direction }\end{array}$ & $\begin{array}{c}\text { Wave } \\
\text { Velocity } \\
\mathbf{V}(\mathbf{m} / \mathbf{s})\end{array}$ & $\begin{array}{c}\text { Normalized } \\
\text { Transmitted Load } \\
\mathbf{p e r} \mathbf{m}(\% / \mathbf{m})\end{array}$ \\
\hline 1 & 0.1 & 2 & 5 & vertical & 1014 & 2.464 \\
\hline 1 & 0.2 & 2 & 5 & vertical & 518 & 1.083 \\
\hline 1 & 0.1 & 2 & 5 & horizontal & 1217 & 0.643 \\
\hline 1 & 0.2 & 2 & 5 & horizontal & 549 & 0.142 \\
\hline
\end{tabular}

Table 5.4 Simulation results for the weakly anisotropic assembly 
The average wave velocities for S-2, M-2 and W-2 in the two directions of wave propagation were found to be 1064,1187 and $1115 \mathrm{~m} / \mathrm{s}$ respectively for $0.1 \mu \mathrm{m}$ interparticle gap spacing case. Porosities of these assemblies were found to be 31.70 , 21.46 and $22.36 \%$ respectively. It can be concluded from these results that wave velocity decreases with increasing porosity. This is supported by experimental measurements of Wyllie et al. (1962), see Fig. 5.36.

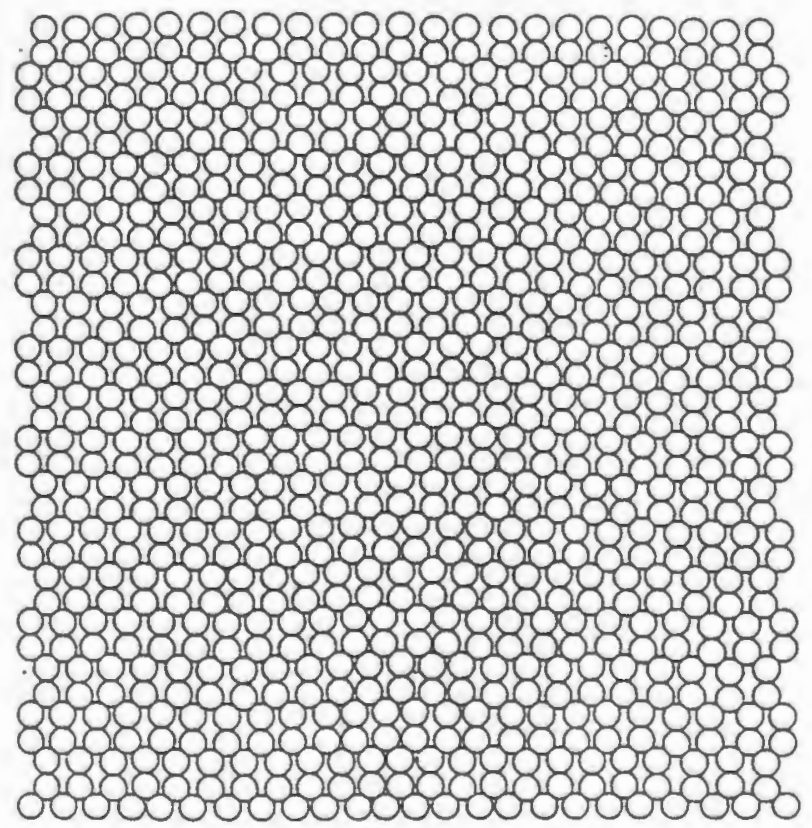

Fig. 5.6 A strongly anisotropic assembly (S-1) 


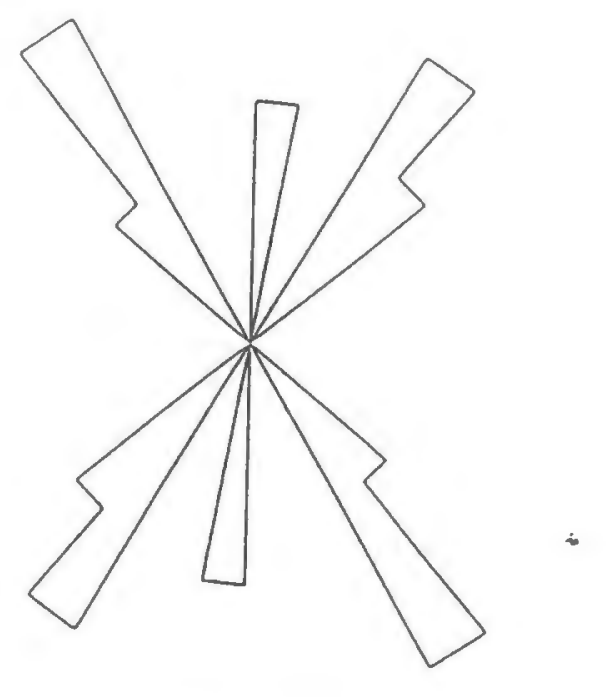

Fig. 5.7 Polar distribution plot for S-1 assembly

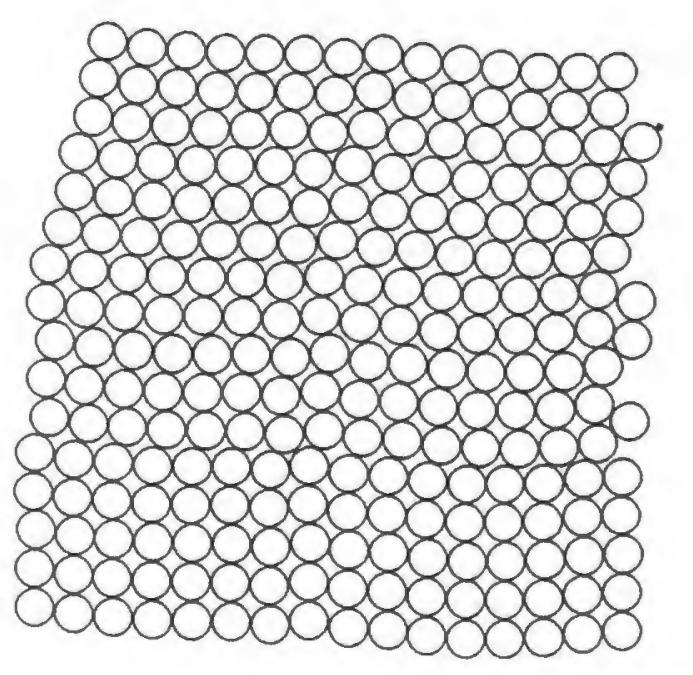

Fig. 5.8 A moderately anisotropic assembly (M-1) 


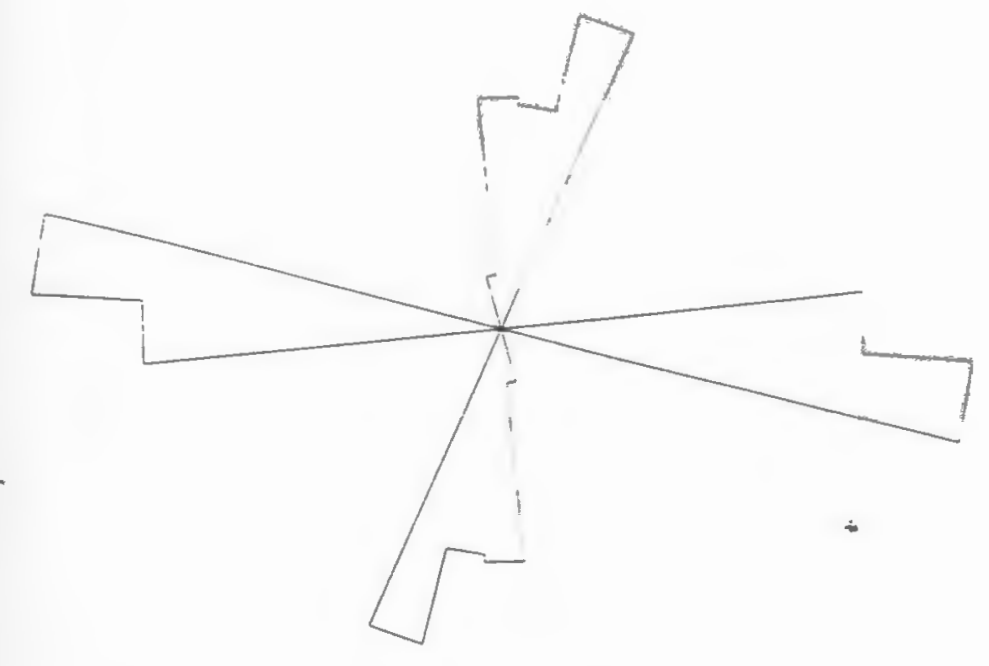

Fig. 5.9 Polar distribution plot for M-1 assembly

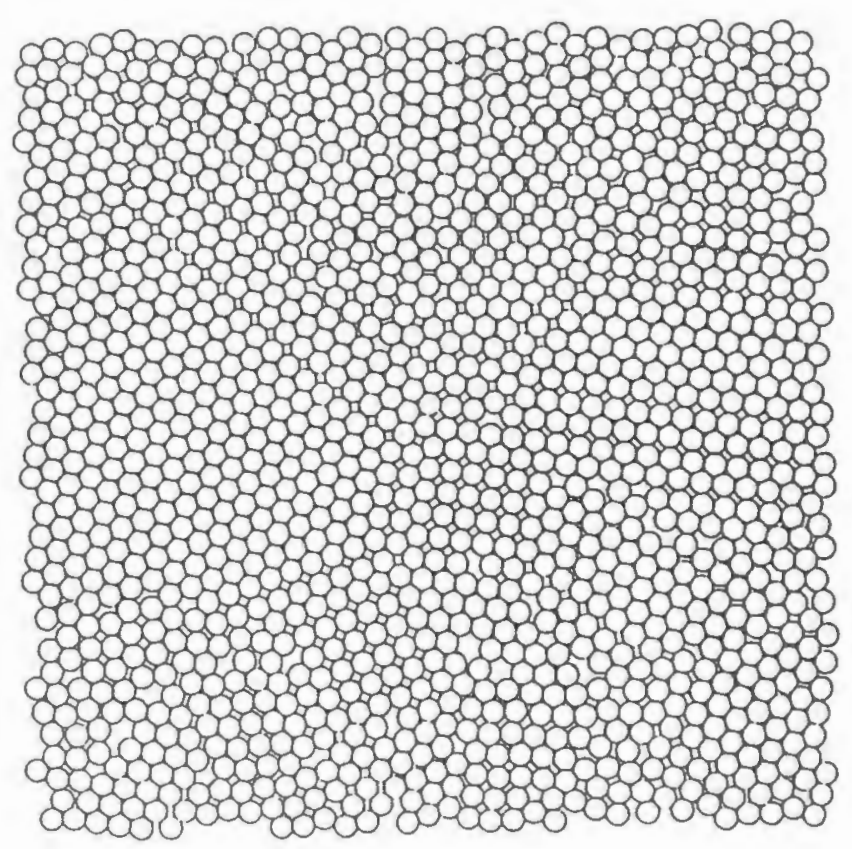

Fig. 5.10 A weakly anisotropic assembly (W-1) 


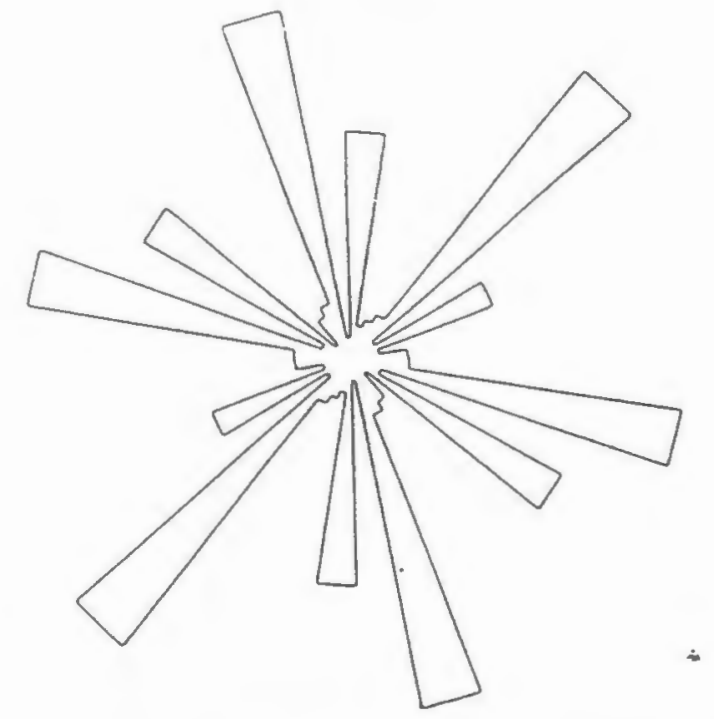

Fig. 5.11 Polar distribution plot for W-1 assembly

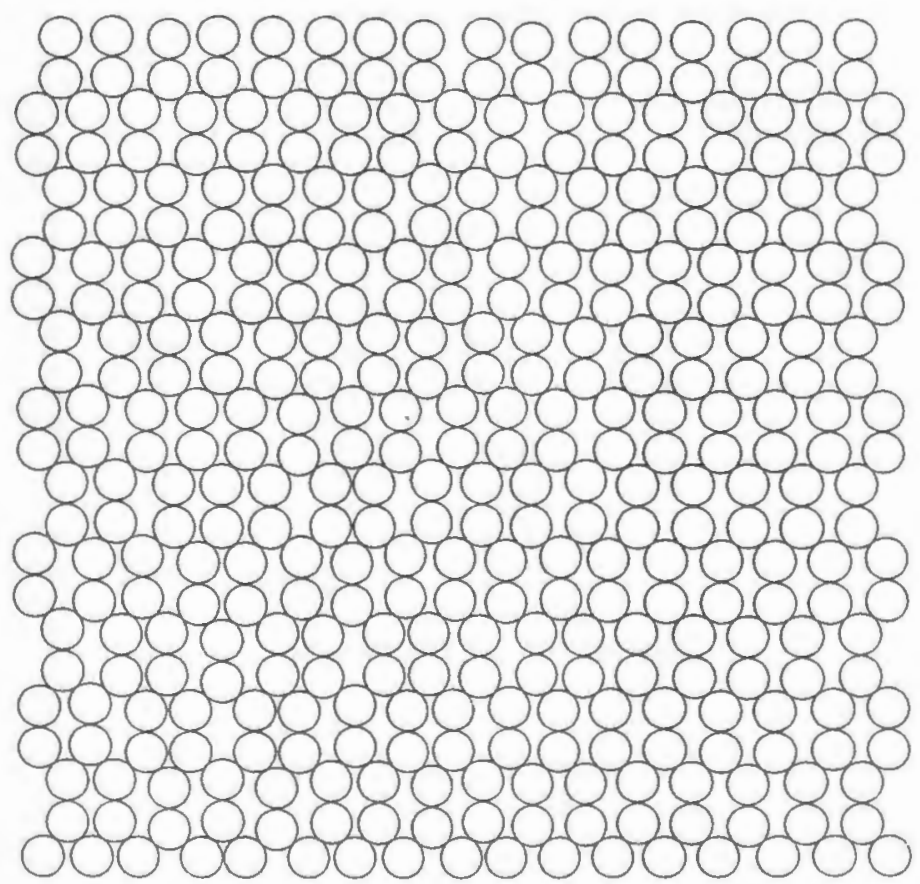

Fig. 5.12 Strongly anisotropic assembly (S-2) used for simulation 


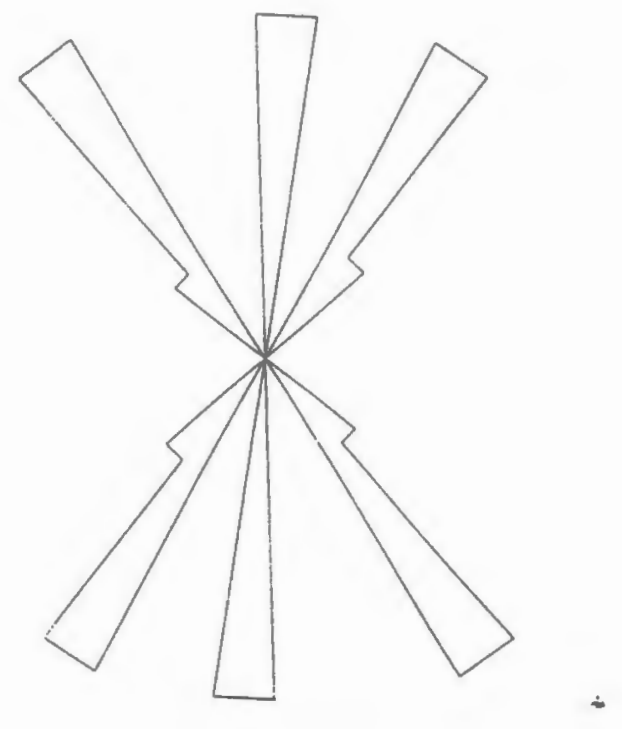

Fig. 5.13 Polar plot of branch vector distribution for S-2

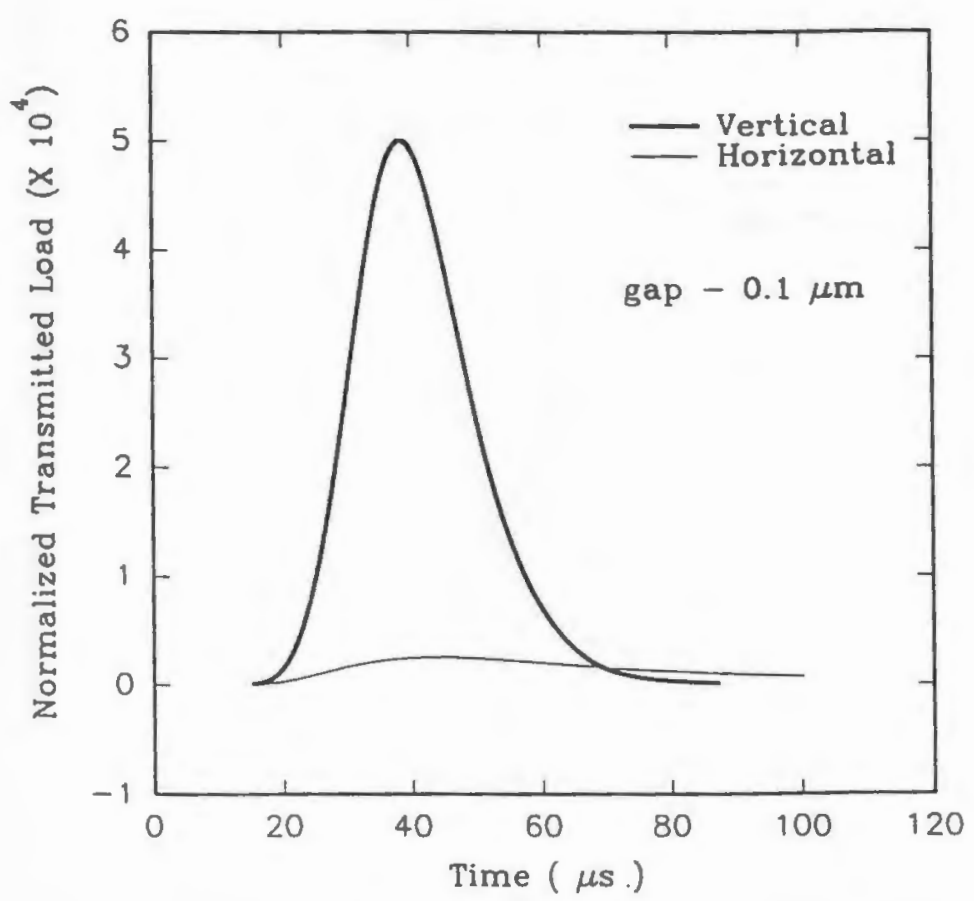

Fig. 5.14 Average normalized transmitted load in two directions for S-2 


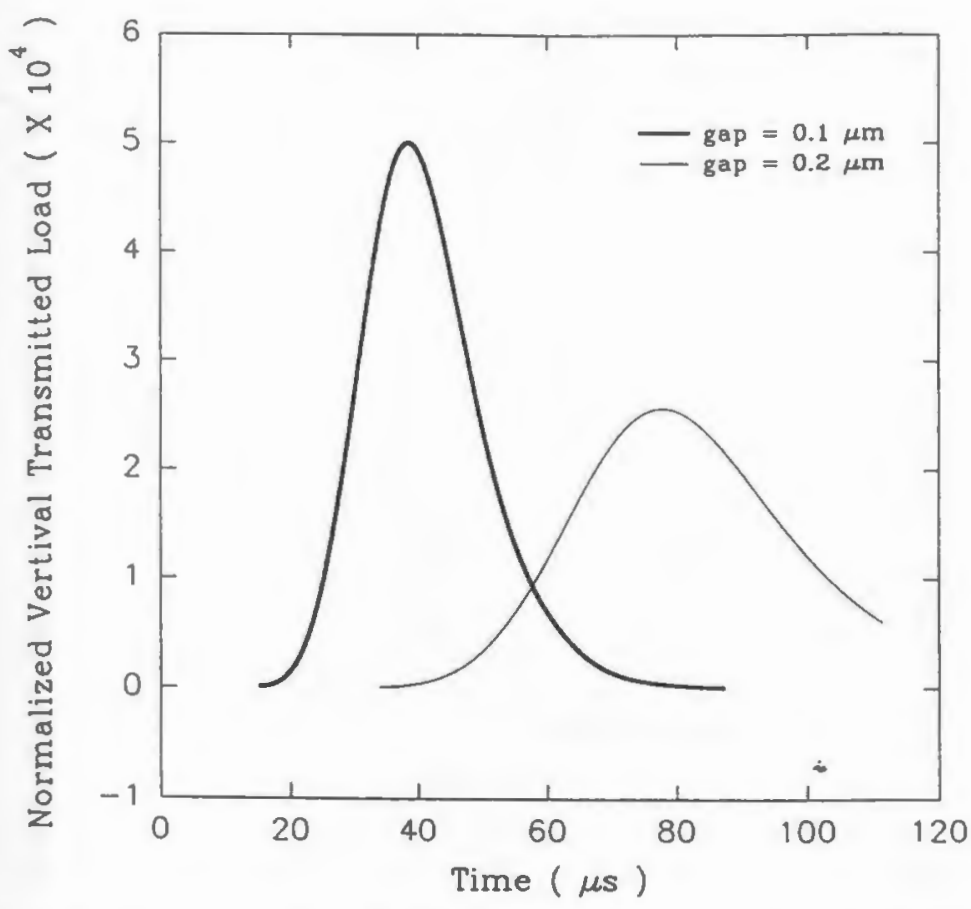

Fig. 5.15 Vertical transmission vs. time at different interparticle spacings for S-2

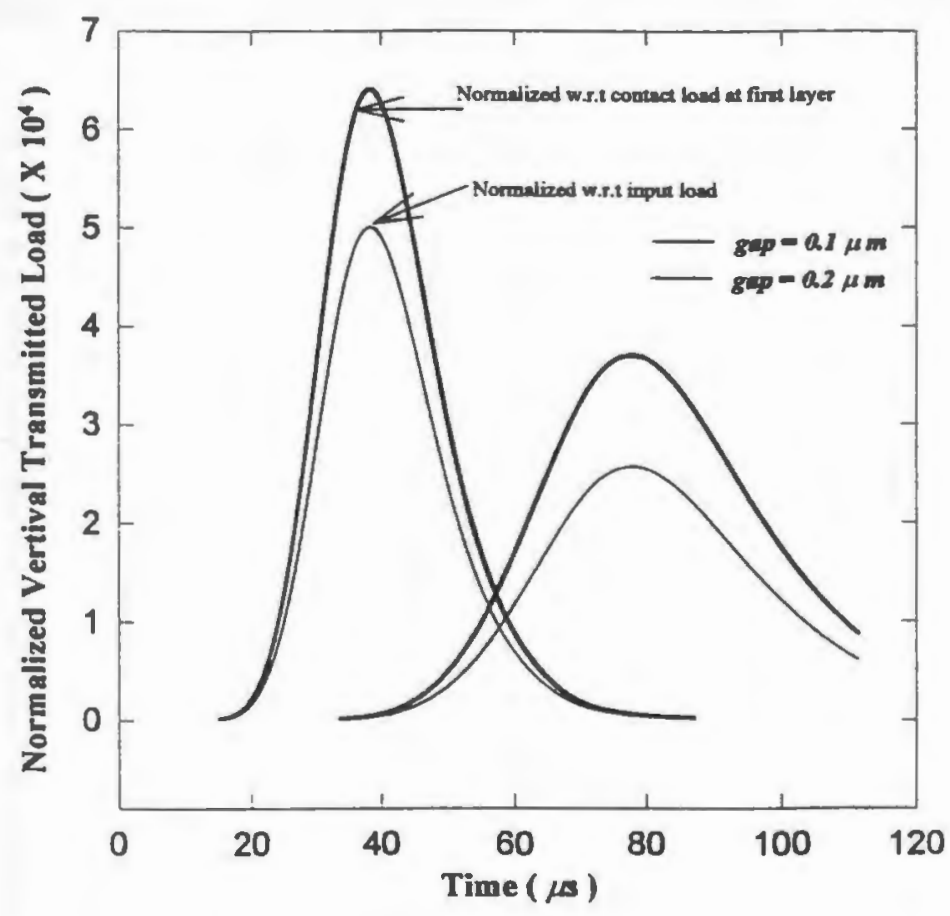

Fig. 5.16 Comparison of vertical transmission normalized w.r.t contact load at first layer and the input load 


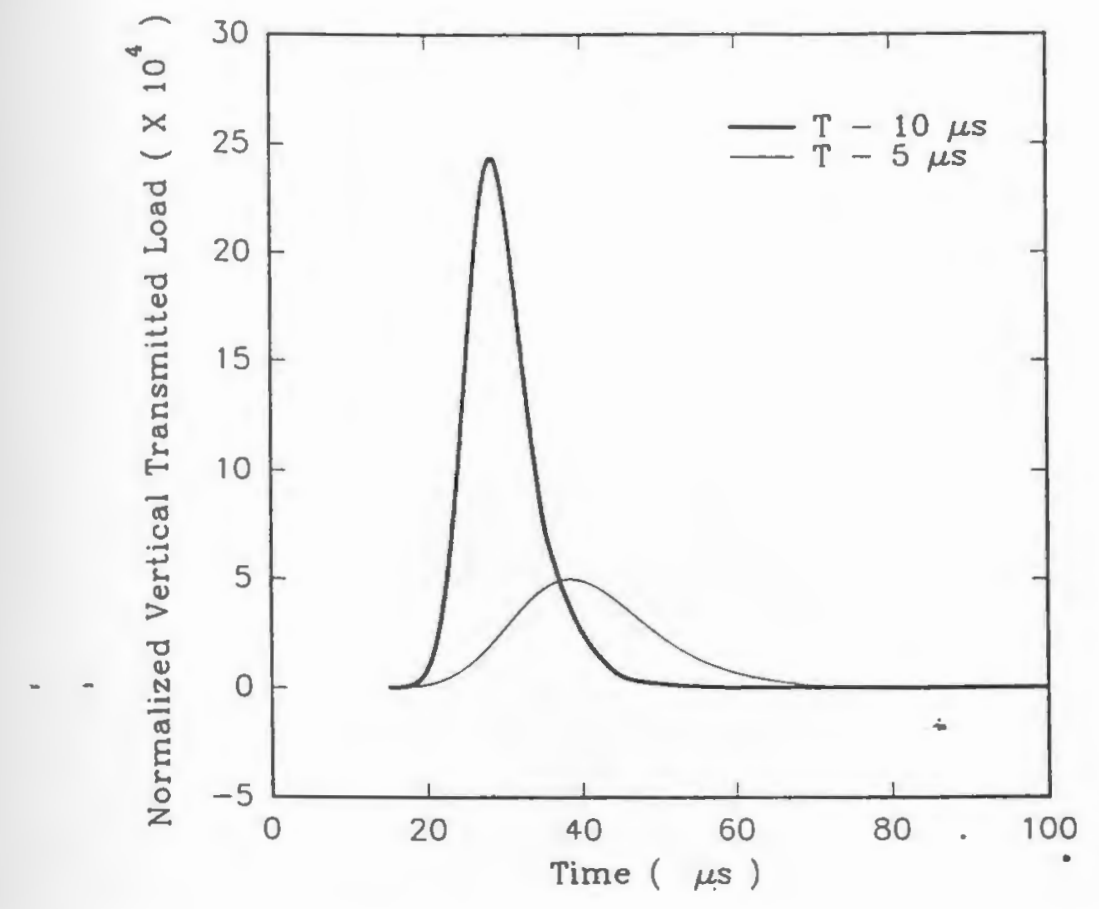

Fig. 5.17 Average normalized contact force for 5 and $10 \mu$ s pulse durations for S-2

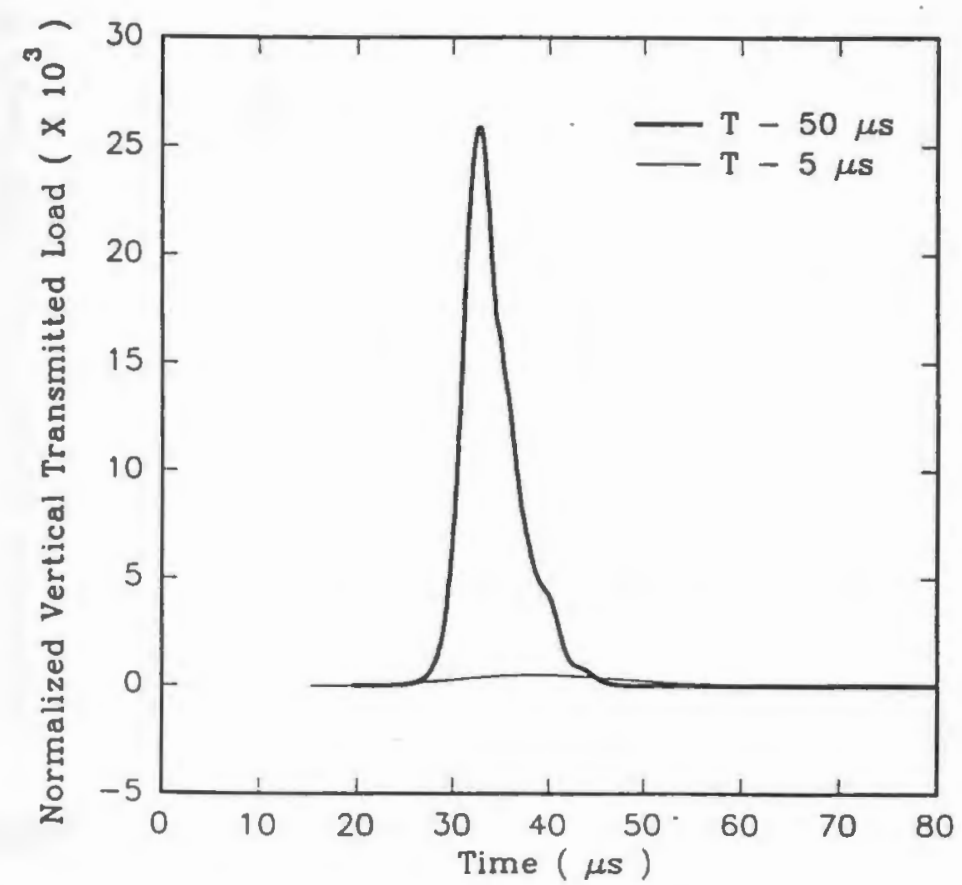

Fig. 5.18 Average normalized contact force for 5 and $50 \mu$ s pulse durations for S-2 


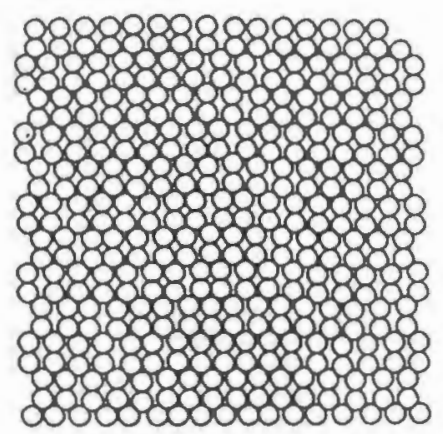

Fig. 5.19 Strongly anisotropic assembly $\mathrm{S}-3$ (radius $=0.5 \mathrm{~mm}$ )

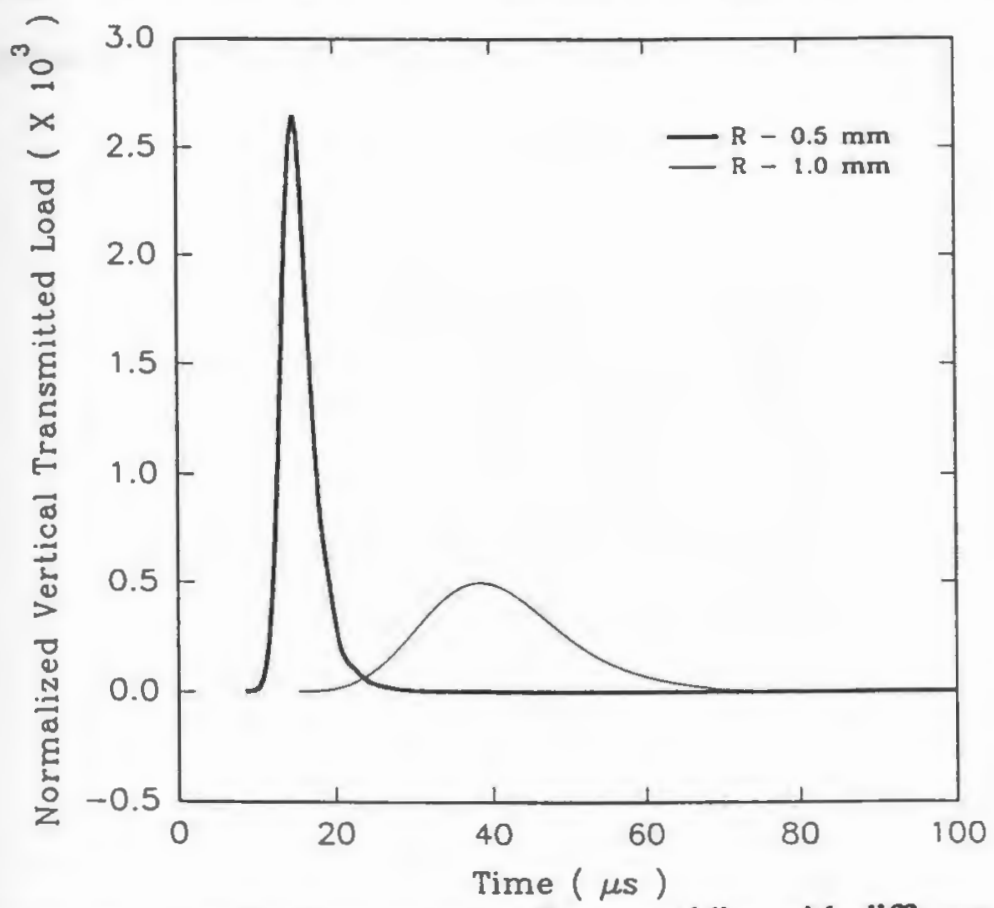

Fig. 5.20 Companson of vertical transmission for assemblies with different sized particles (constant input load) 


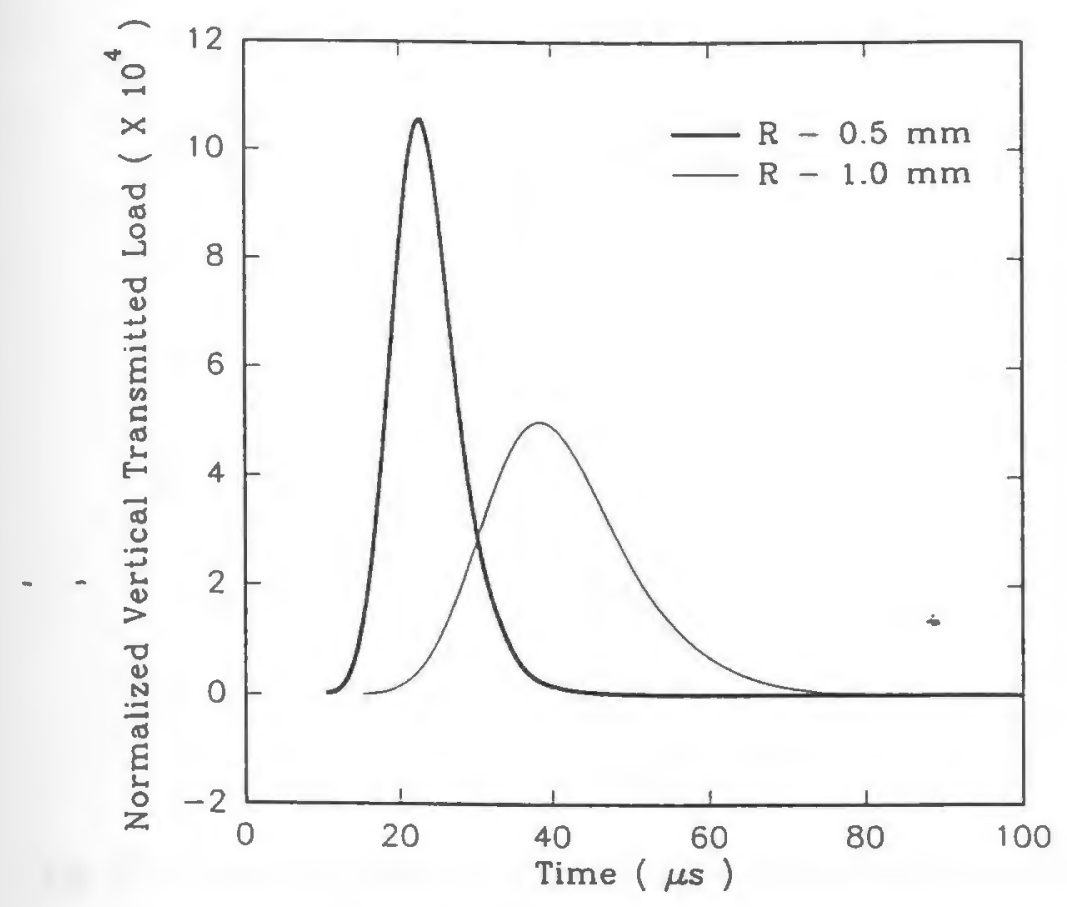

Fig. 5.21 Comparison of vertical transmission for assemblies with different sized particles (constant input stress)

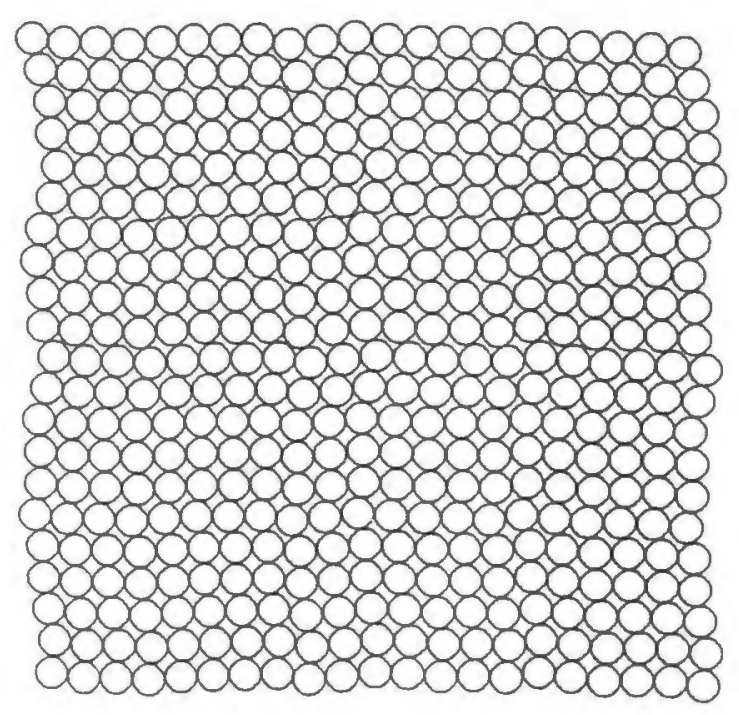

Fig. 5.22 Moderately anisotropic assembly $M-2$ with particle radius $R=1 \mathrm{~mm}$ 


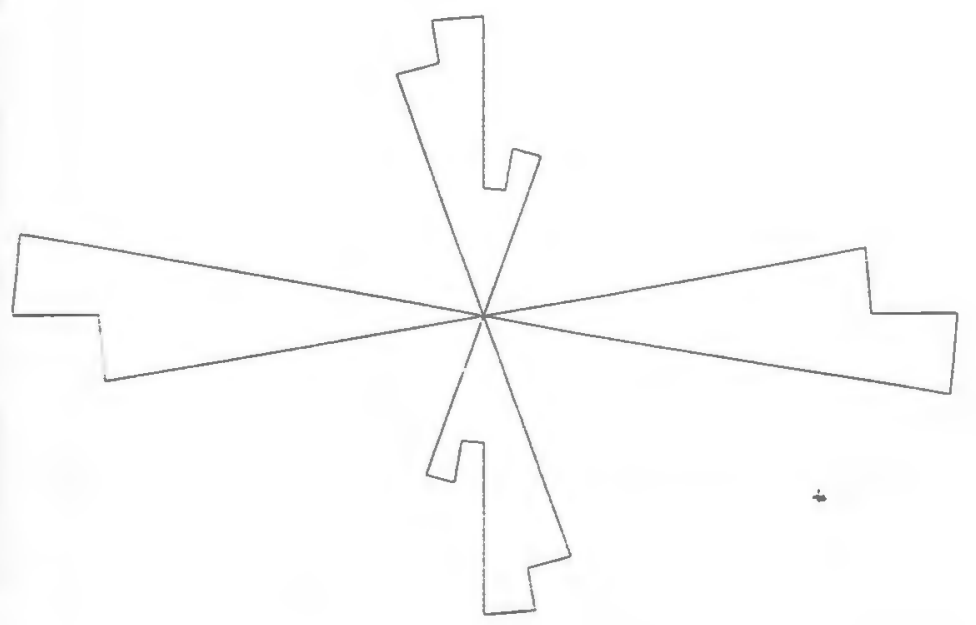

Fig. 5.23 Polar plot of branch vector distribution for $\mathrm{M}-2$ assembly

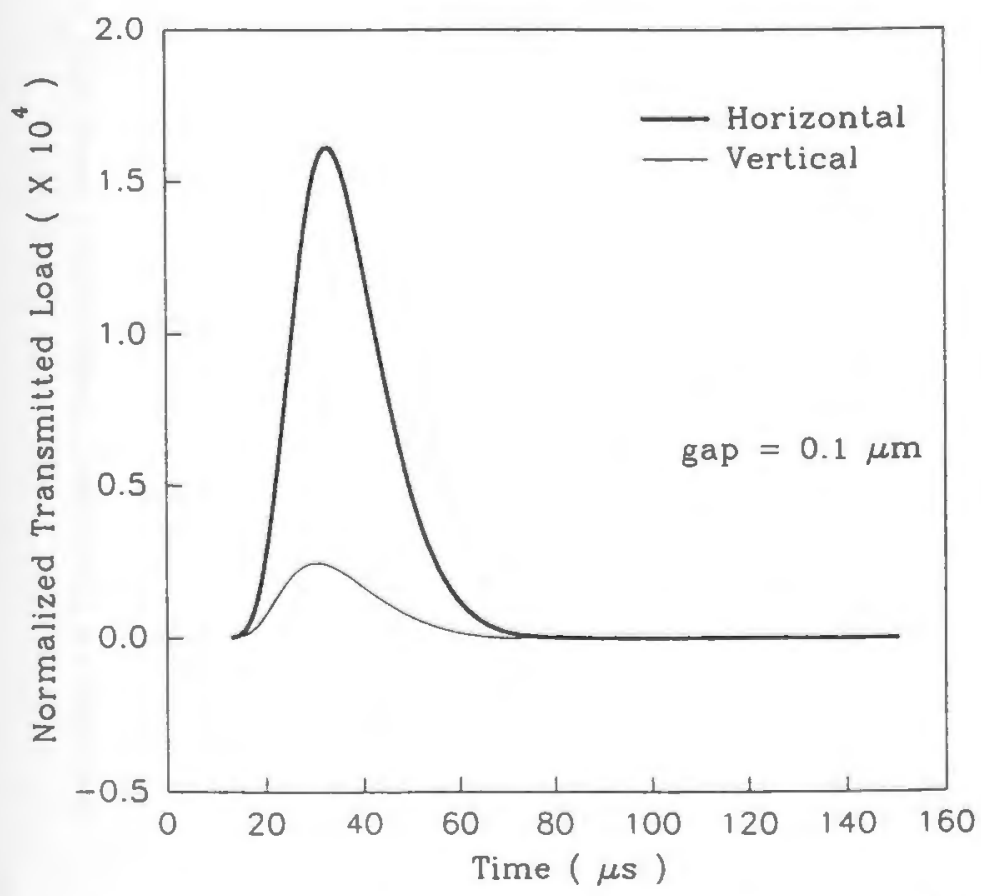

Fig. 5.24 Horizontal and vertical transmission through M-2 


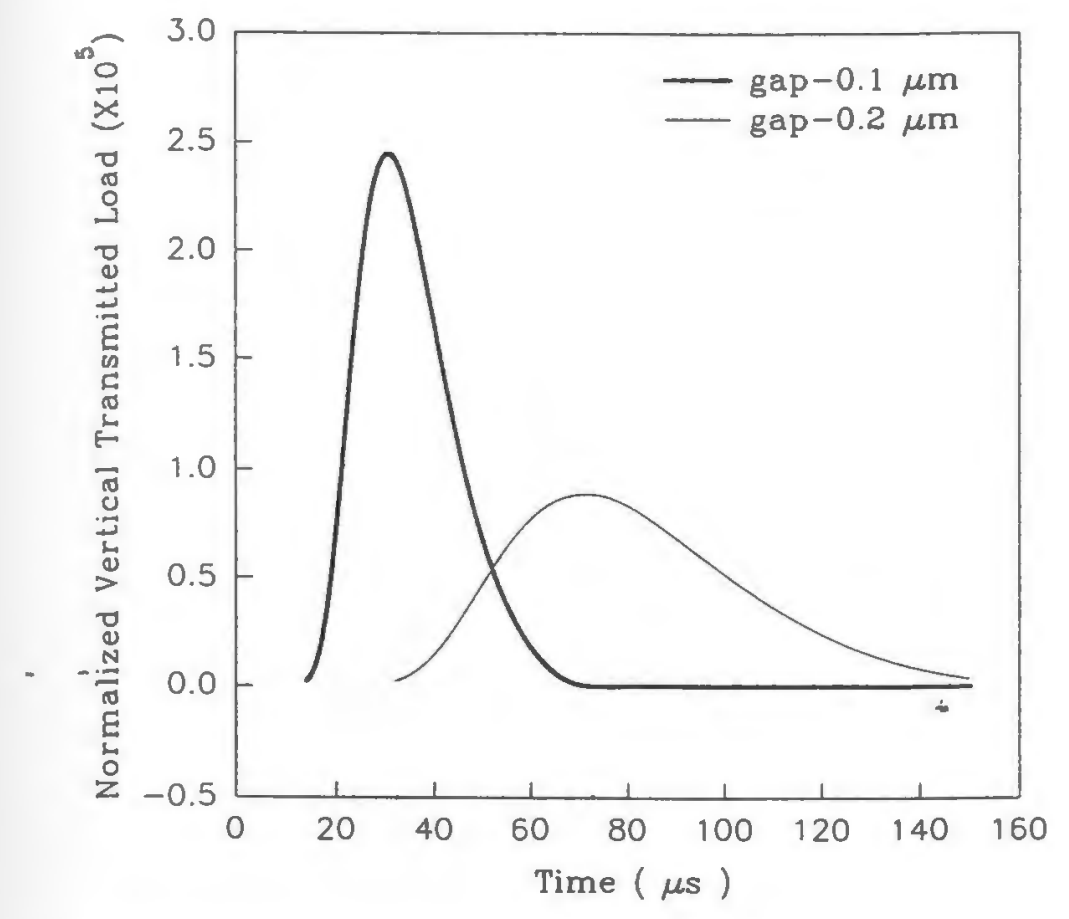

Fig. 5.25 Vertical transmission through $\mathrm{M}-2$ for different interparticle gap spacing

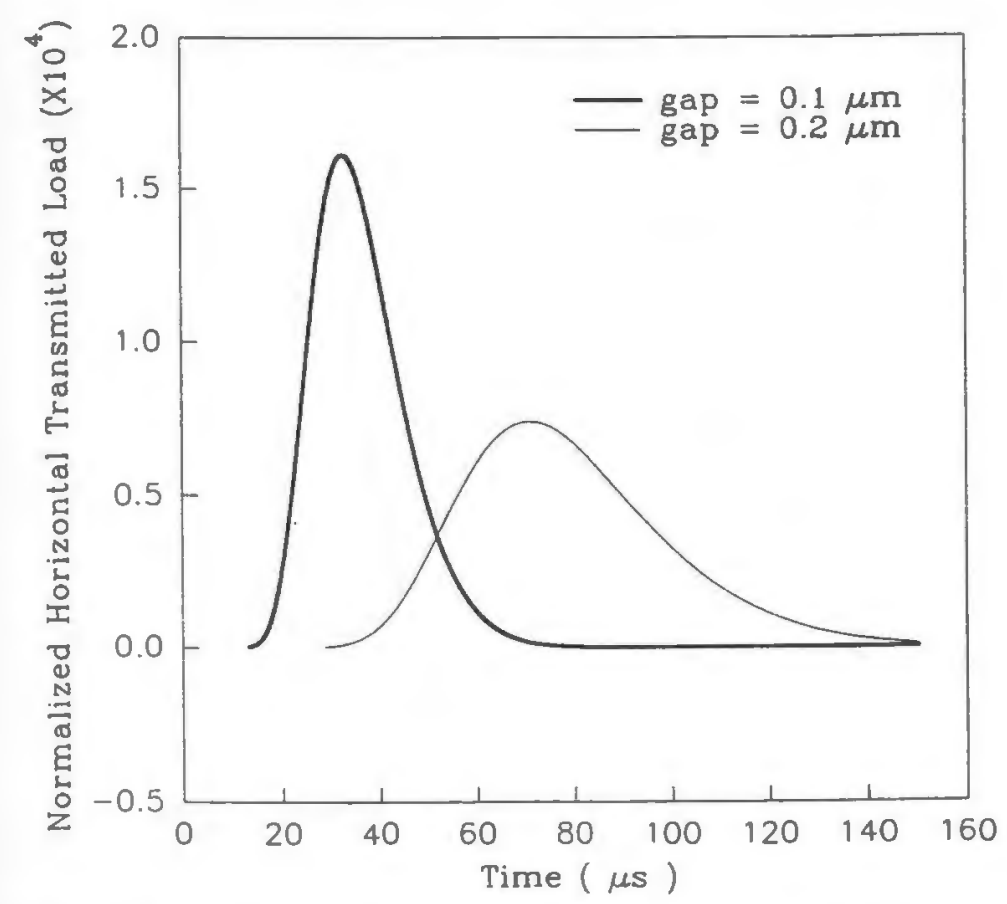

Fig. 5.26 Horizontal transmission through M-2 for different interparticle gap spacing 


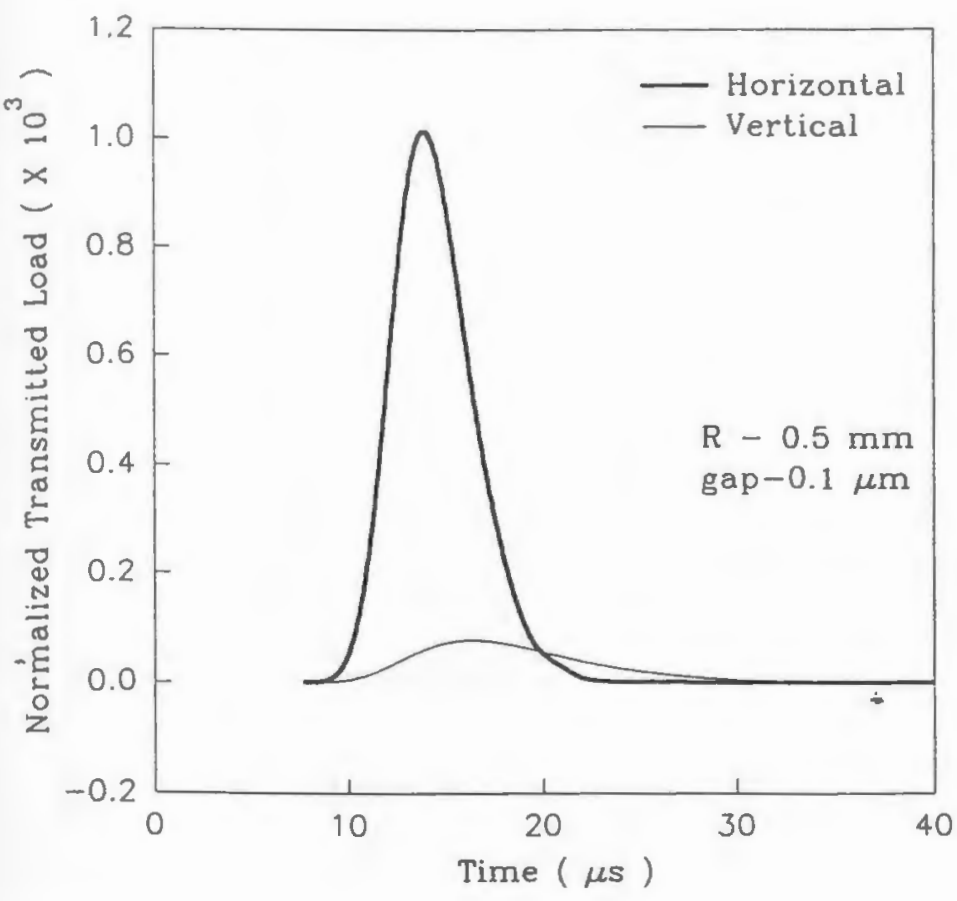

Fig. 5.27 Comparison of horizontal and vertical transmission for $\mathrm{M}-2$ (radius $=0.5 \mathrm{~mm}$ )

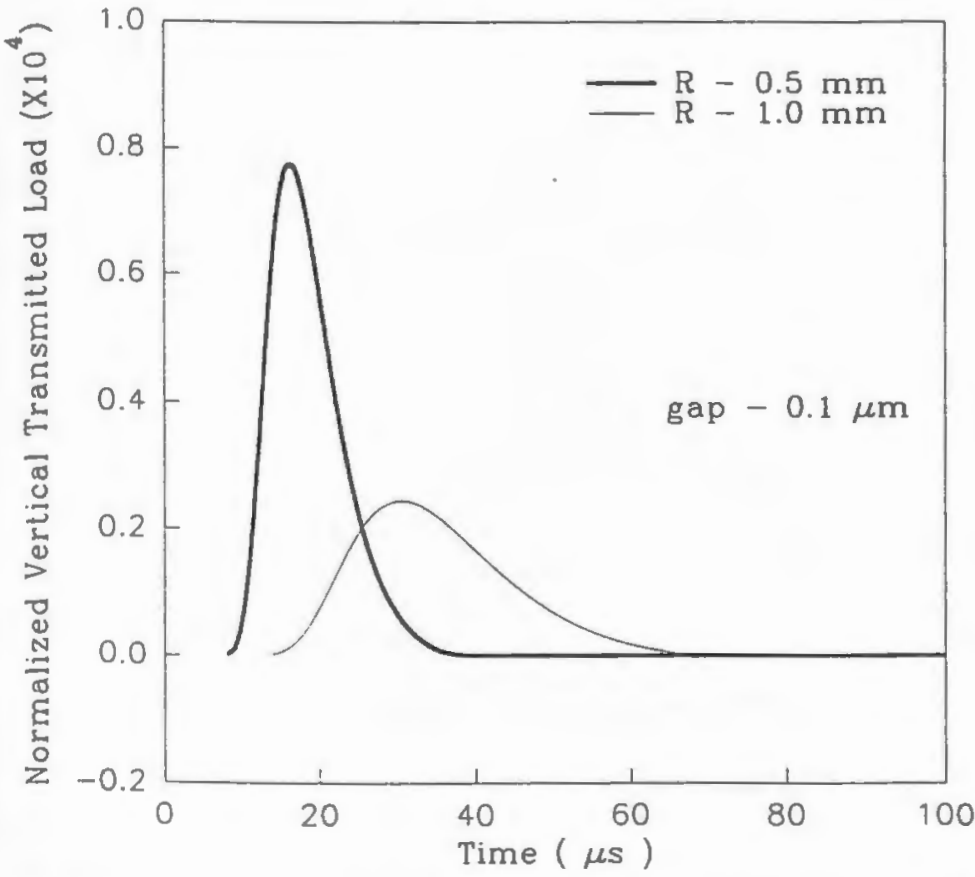

Fig. 5.28 Vertical transmission for assemblies with different sized particles 


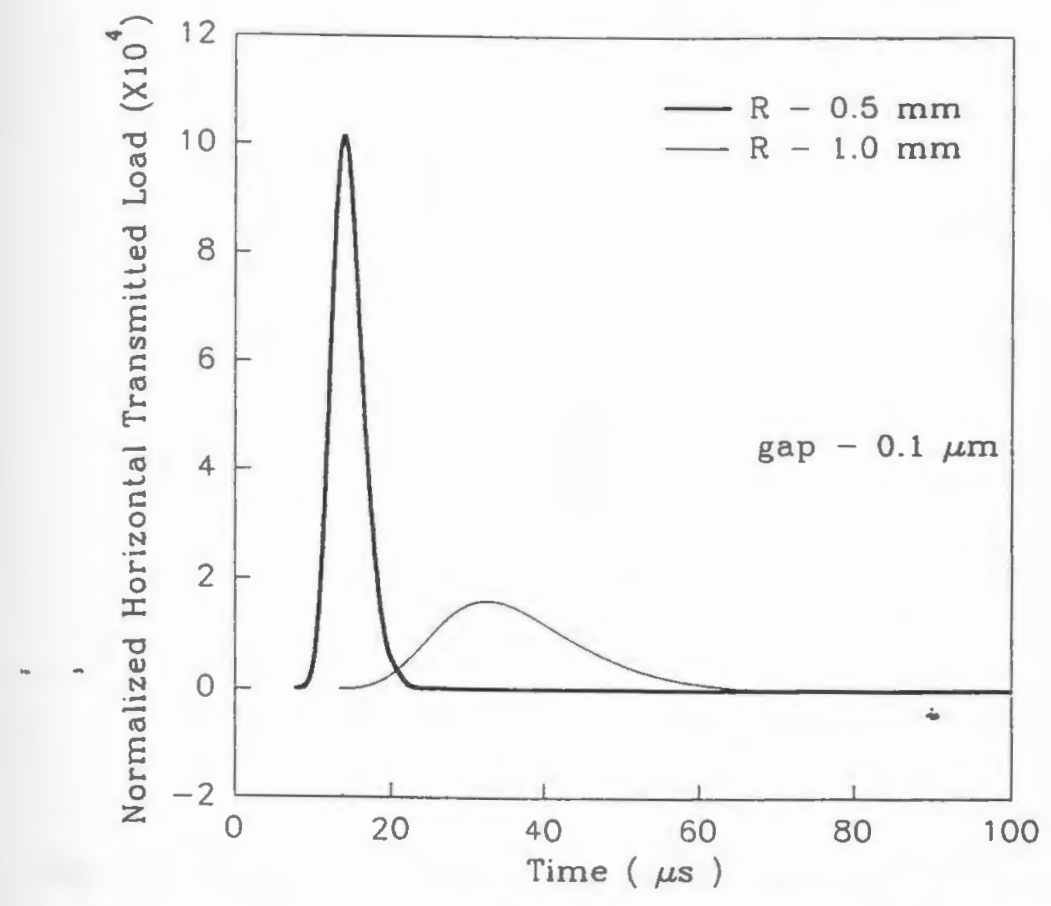

Fig. 5.29 Horizontal transmission for assemblies with different sized particles

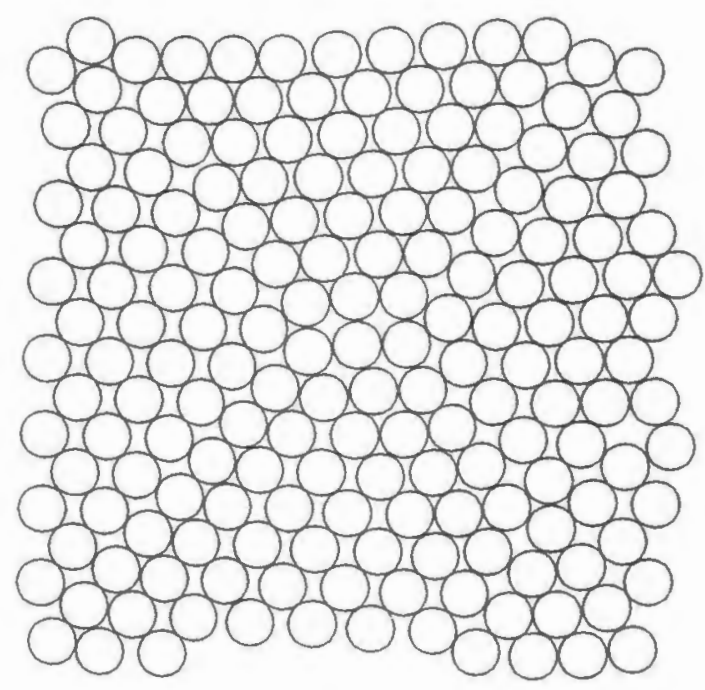

Fig. 5.30 Weakly anisotropic assembly W-2 with particle radius $=1 \mathrm{~mm}$ 


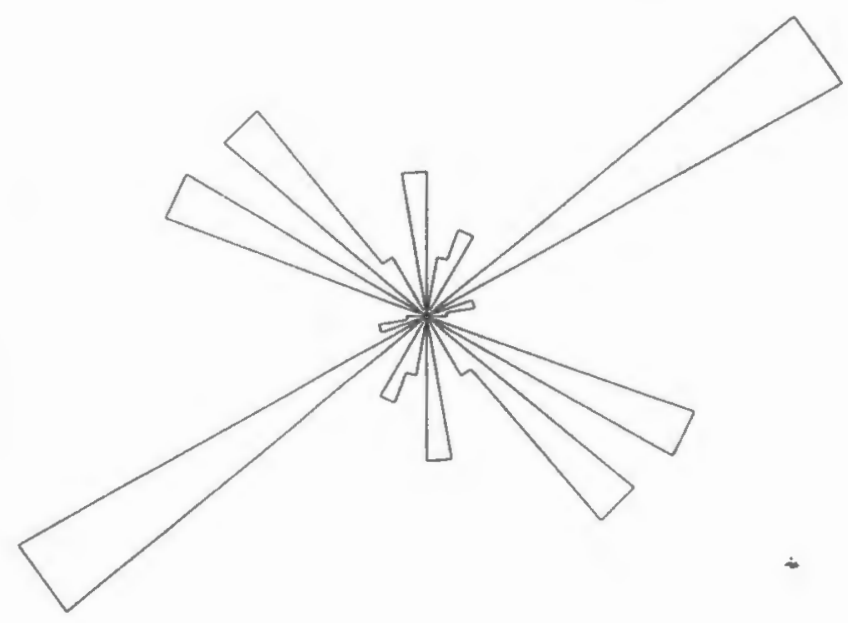

Fig. 5.31 Polar plot of branch vector distribution for W-2 assembly

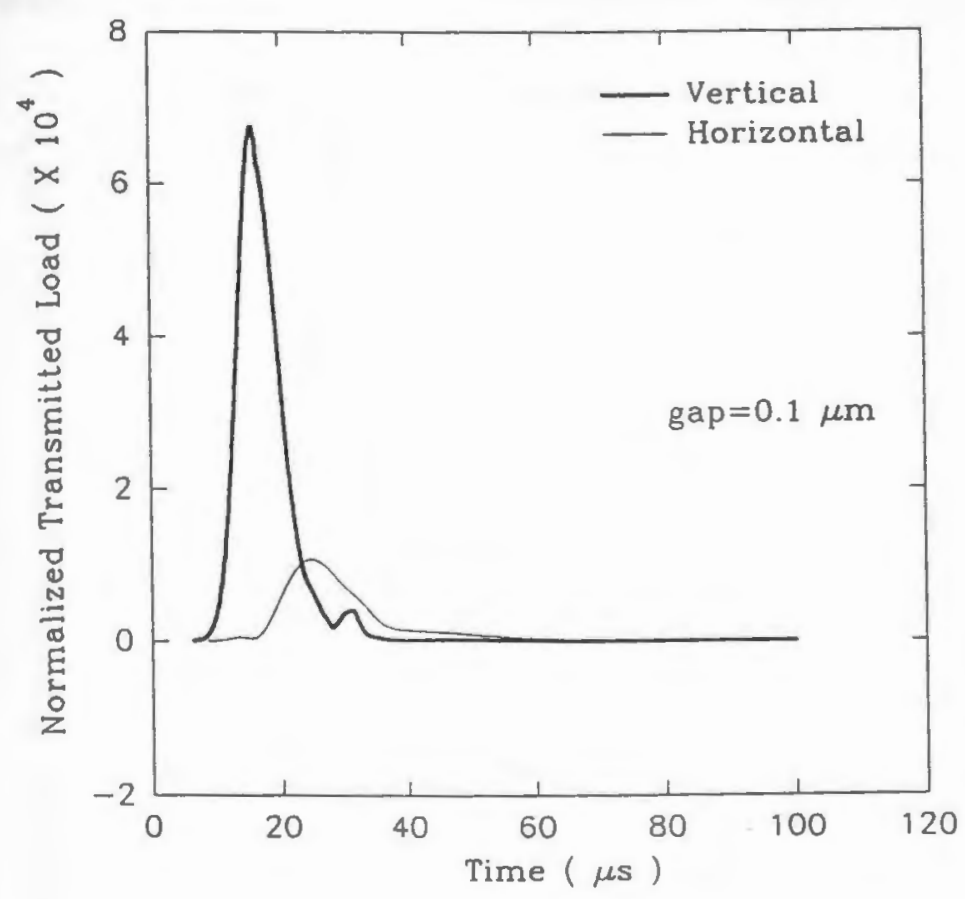

Fig. 5.32 Average transmitted load vs. time for horizontal and vertical directions for W-2 (interparticle gap spacing $=0.1 \mu \mathrm{m}$ ) 


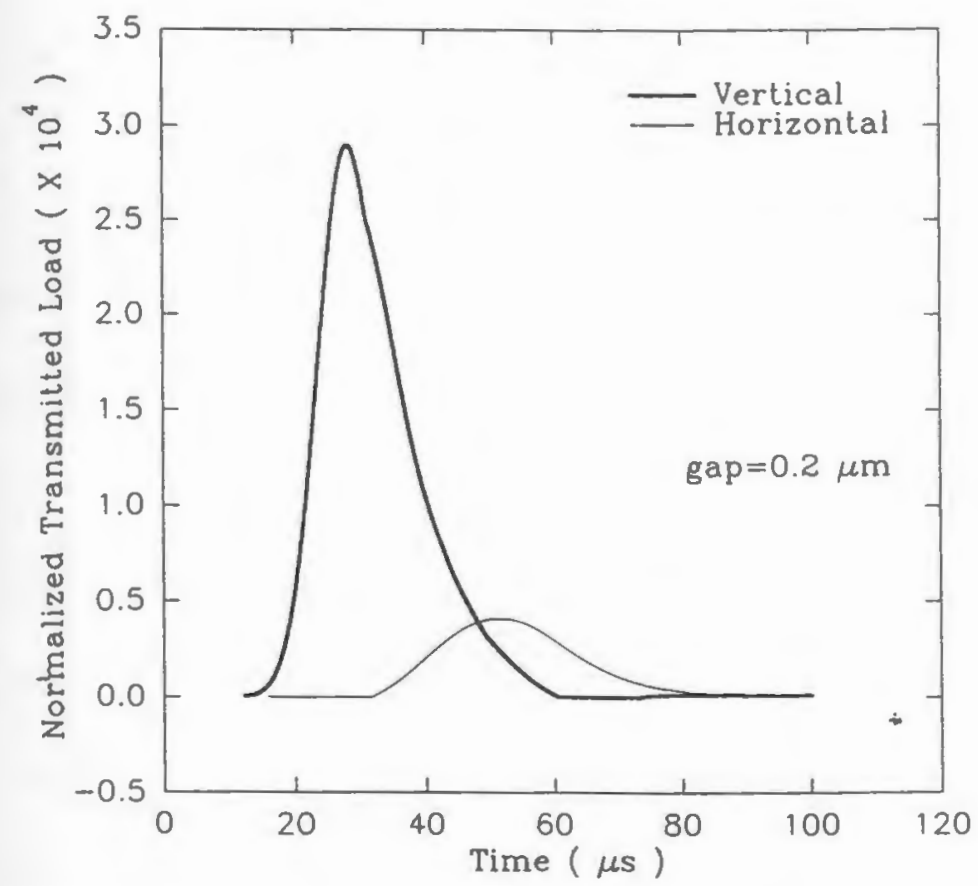

Fig. 5.33 Average transmitted load vs. time in horizontal and vertical directions for W-2

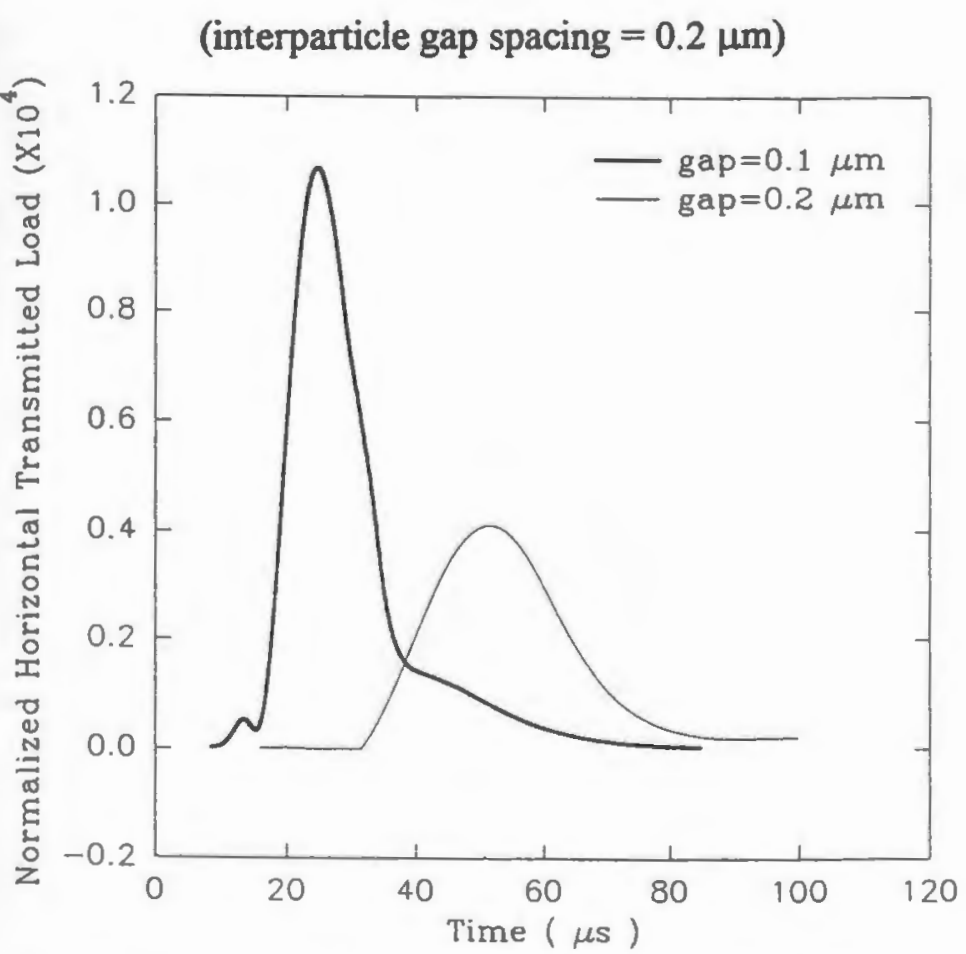

Fig. 5.34 Horizontal transmission for different interparticle spacings in W-2 


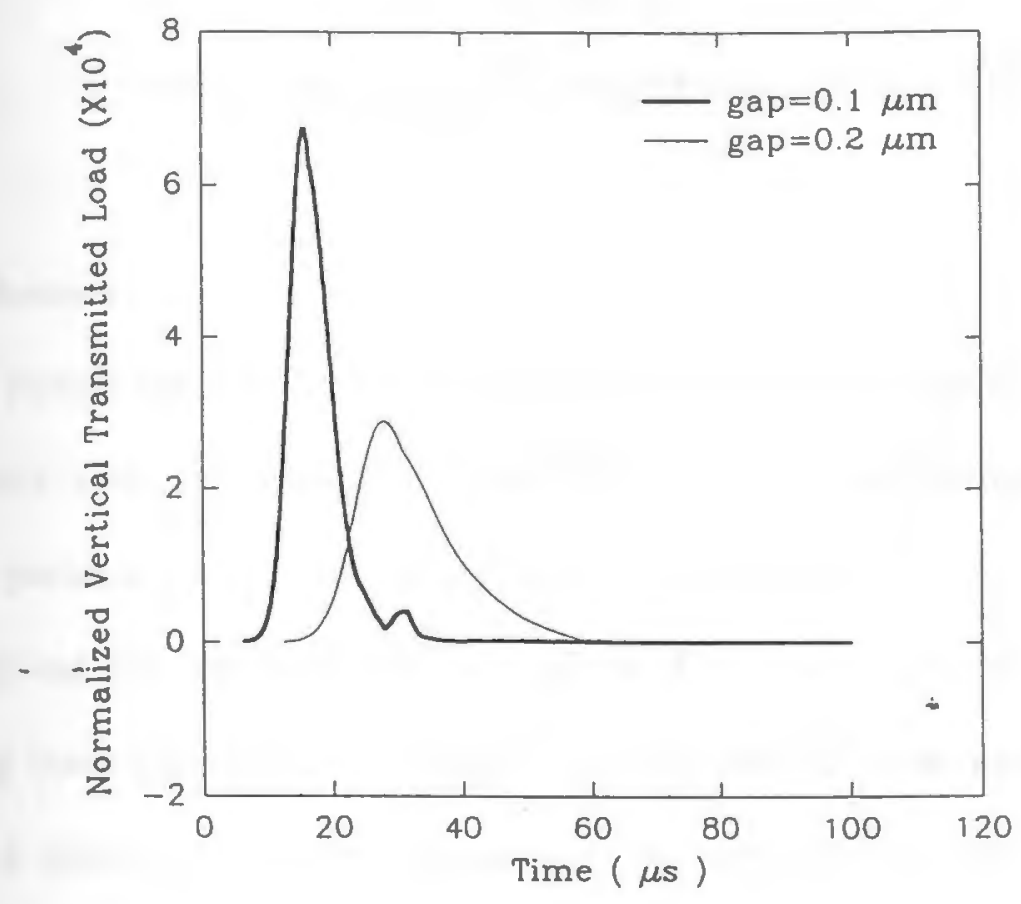

Fig. 5.35 Vertical transmission for different interparticle spacings in W-2

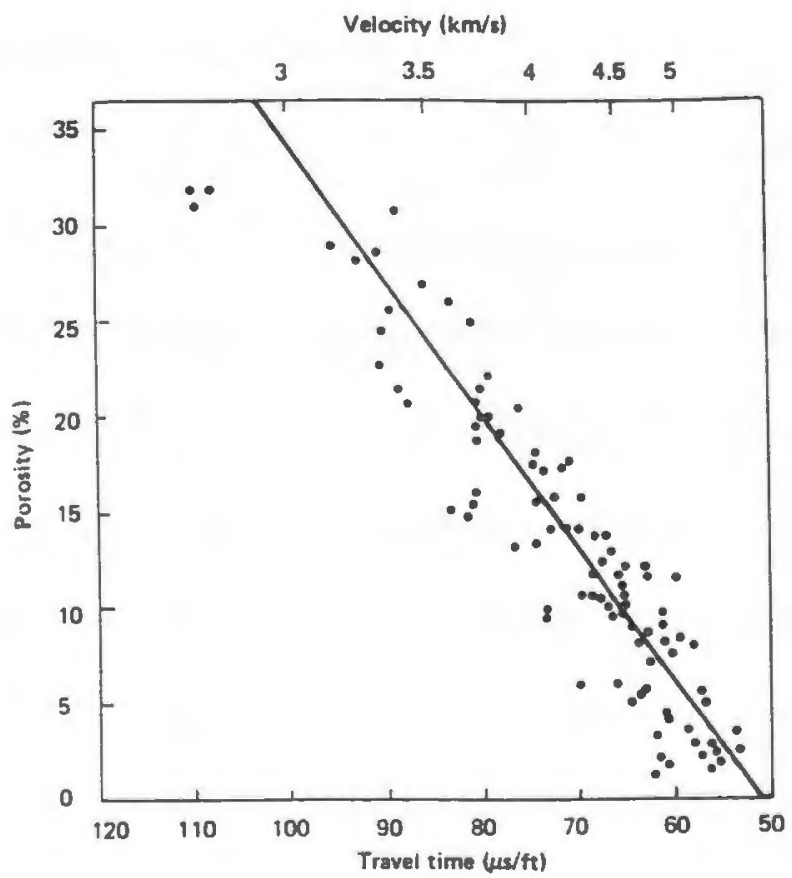

Fig. 5.36 Wave velocity/porosity relationship (Wyllie et al., 1962) 


\section{Chapter 6}

\section{CONCLUSIONS AND RECOMMENDATIONS}

\subsection{Conclusions}

A particle contact theory based upon elastohydrodynamics modeling has been used to construct a discrete element computer code to study wave propagation in fluidsaturated particulate media. This theory identifies a mechanism related to the presence of pore fluid and the permeability of the medium. The theory was used for numerically simulating wave propagation and dynamic load transfer through saturated particulate media. An attempt was made to study the effects of pulse-duration or wave-length, particle size, fluid viscosity, interparticle gap spacings, etc. on the wave propagational behaviors through porous materials. Studies also correlated the model material's microstructure or fabric to dynamic behavior.

It was observed that the presence of pore fluid can have significant effects on the macroscopic dynamic behaviors of particulate materials by changing the contact response between adjacent particles through hydrodynamic squeeze-film action. The interparticle gap spacing has significant effects on wave attenuation as well as wave-velocity. However pore fluid viscosity does not show much influence on wave attenuation. Attenuation increases slightly with increasing pore fluid viscosity, and it is supported by experimental results (Jones and Nur, 1983). It was found that there was a slight increase in wave speed with increasing pore fluid viscosity. Wave speed was also found to be higher through assemblies of stiffer particles. For the one-dimensional case, the dispersive behavior 
depends upon the wavelength to particle size ratio. A long pulse duration or wavelength input wave decomposes into a series of short propagating pulses (ringing phenomenon), which is consistent with the results found by Shukla et al (1993) for dry one-dimensional case.

For two-dimensional assembly, more wave transmission was observed in the preferred direction, i.e. the direction of more branch vector distribution for model material system. Wave attenuates less and wave velocity is greater in the preferred direction. It was shown that with an increase of interparticle gap spacing, attenuation increases while wave velocity decreases. Wave speed was found to be higher, and so was the transmitted load per unit of distance travelled for smaller particle (i.e. particle of diameter $1 \mathrm{~mm}$ ) than for particles with $2 \mathrm{~mm}$ diameter. These results were consistent for various model assemblies with different anisotropies. Porosities of various assemblies were calculated. Again, it was observed that wave velocity decreases with increasing porosity. Also, it can be concluded that wave propagational behaviors of particulate media are directly related to the microstructure or fabric of them.

\subsection{Recommendations}

With the experience gained in the research work in the numerical analysis of wave propagation through saturated particulate media, several recommendations can be made for further study in this or related fields.

First, particles were assumed to be circular disks in the present work, whereas in reality, they are more like irregular shaped. So, numerical studies should be conducted 
with assemblies of non-circular particles, say for example elliptical-shaped particles. Moreover, three-dimensional arrays of particles should be generated and analyzed to more closely simulate real granular materials.

Several assumptions were made in developing the elastohydrodynamic contact law for the interaction between pore fluid and solid skeleton. Viscosity of the pore fluid was assumed to be constant in this work. Future work in this field should incorporate the effect of temperature, pressure and shearing rate on pore fluid viscosity. Also, fluid inertia should be taken into account for developing an appropriate contact model for the coupled mechanical response between fluid and solid phase. A more appropriate tangential law should be considered for better modeling of shearing between two particles, including slip velocity at boundary surfaces at very small interparticle gap spacings.

Efforts should be made to correlate wave propagation characteristics to other fabric measures, such as normal contact vectors, coordination number, path fabric, void polygon fabric etc. The meso-domain approach applied to assemblies with larger numbers of particles would be helpful in passing from microscopic characteristics to macroscopic behavior of particulate media.

Considerable computer memory space and CPU time are needed to study wave motion in more realistic particulate media. To model real particulate media, threedimensional assembly generators would be required. 


\section{Appendix A}

\section{NUMERICAL COMPUTER CODE}

The discrete element numerical code structure used for simulating wave propagation and dynamic load transfer in saturated granular media is given in Fig. A.1. The entire code is listed in the following pages.

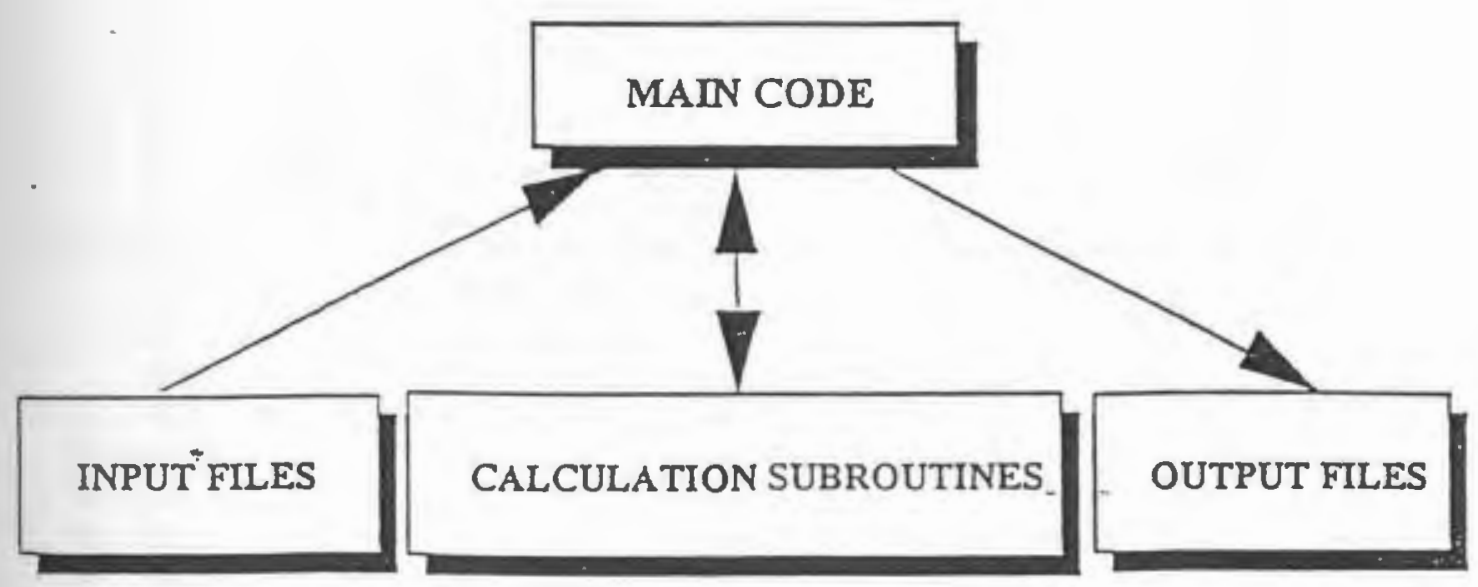

Fig. A.1 Numerical computer code structure 
THIS CODE IS USED TO INVESTIGATE WAVE PROPAPGATION

$\mathrm{C}$

GAUTAM ADHIKARI \& QIMING TAI

C

C DEPARTMENT OF MECHANICAL ENGINEERING \& APPLIED MECHANICS

C UNIVERSITY OF RHODE ISLAND

C DESCRIPTION OF MAIN VARIABLES:

C $\quad \mathrm{X}, \mathrm{Y}$-COORDINATES OF DISK CENTERS

C U,V-VELOCITIES OF DISK CENTERS

C

W-ROTATIONAL VELOCITY OF DISK

R-_-RADIUS OF DISK

DUI_ RELATIVE DISPLACEMENT OF DISK CENTERS

NCT__CONTACT INFORMATION OF DISKS

NDEX-TOTAL NUMBER OF EXPLOSIVE FORCE C

PNEX-NORMAL PEAK VALUE OF EXPLOSIVE FORCE

PSEX-TANGENTIAL PEAK VALUE OF EXPLOSIVE FORCE C

ANG-ANGLE OF EXPLOSIVE FORCE C

EXTB-TTME THE EXPLOSION BEGINS

EXTE--TMME THE EXPLOSION ENDS

KN,KS-CONTACT STIFFNESS C

M-MASS OF DISKS

NO-MASS DENSITY OF DISK $\quad C$

TH-THICKNESS OF DISKS

PO-POISSON'S RATIO C

E-_-YOUNG'S MODULUS C

FN-_-NORMAL CONTACT FORCE C

C FS-TANGENTIAL CONTACT FORCE C

IMPLICIT DOUBLE PRECISION(A-H,O-Z)

REAL KN,M,NO,KS,IZ,px(2),py(2)

DIMENSION X(1000,2),Y(1000,2),U(1000,2), V(1000,2),

$\% \quad \mathrm{PO}(10), \mathrm{E}(10), \mathrm{W}(1000,2), \mathrm{Xl}(1000), \mathrm{Y} 1(1000), \mathrm{R}(10), \mathrm{NO}(10)$,

$\% \quad \operatorname{SI}(1000,0: 10), \operatorname{CO}(1000,0: 10), \operatorname{DI}(1000,10), \operatorname{NCT}(1000,0: 10), \operatorname{NDEX}(20)$,

$\% \quad \operatorname{PNEX}(20), \operatorname{PSEX}(20), \operatorname{ANG}(20), \operatorname{EXTB}(20), \operatorname{EXTE}(20), \operatorname{KN}(10,10), \mathrm{M}(10,10)$,

$\% \operatorname{BK}(10,10), \operatorname{BKS}(10,10), \mathrm{FSW}(1000,0: 10,2), \mathrm{NG}(1000,40), \mathrm{FNM}(1000,40)$,

$\% \operatorname{TFNM}(1000,40), \operatorname{KS}(10,10), \operatorname{DTM}(10,10), \operatorname{DTIZ}(10,10), \operatorname{IZ}(10,10)$,

$\% \quad \operatorname{FNW}(1000,0: 10,2), \operatorname{accx}(1000,2), \operatorname{accy}(1000,2), \operatorname{accw}(1000,2)$,

$\% \quad$ nkout $(2,200)$,out l $1(30,50000)$,ttime( 50000$)$

CHARACTER GEOFL*16,PHYFIL *16,EXPFIL *16,CALFL*16,

$\%$ MAINFIL $* 16$, OUTFL *30

COMMON/DT/DT /AA/AA /BB/BB /BETA/BETA /DYN/DYN /AMU/AMU

$/ C 0 / C 0 / N D G / N D G(1000) / N D M / N D M(1000) / T H / T H(10) / N D M M / N D M M$

$\% \quad$ NDGM/NDGM/KKK/K/const/const, CC,visco,HH/DELT/DELT $(1000,10,2)$

$\%$ /nct0/nct0(1000,20)/uvmax/uvmaxf/ACC/ACCX,ACCCY,ACCW/db/delta,bata

C_-WHAT KIND OF ASSEMBLY DO YOU WANT TO DEAL WITH? INPUT FILE C--_NAME OPEN(UNTT=1,FILE='MAINF.TXT',STATUS='OLD')

READ $(1,20)$ GEOFIL

FORMAT(A16) 
CLOSE(1)

C_INPÜT GEOMETRY INFORMATION

OPEN(UNIT $=2$, FILE=GEOFIL,STATUS $=$ 'OLD')

$\operatorname{READ}(2, *) \mathrm{ND}$

$\operatorname{READ}(2, *)(\mathrm{X} 1(\mathrm{I}), \mathrm{Y} 1(\mathrm{I}), \mathrm{NDG}(\mathrm{I}), \mathrm{NDM}(\mathrm{I}), \mathrm{I}=1, \mathrm{ND})$

CLOSE(2)

$\mathrm{DO} \mathrm{I}=1, \mathrm{ND}$

WRITE $\left(6,{ }^{*}\right) \mathrm{X} 1(\mathrm{I}), \mathrm{Y} 1(\mathrm{I})$

END DO

OPEN(UNIT=23,FILE='GEO.DAT, STATUS='OLD')

$\operatorname{READ}(23, *) \mathrm{NDGM}$

READ(23,*) (TH(I),R(I),I=1,NDGM)

CLOSE(23)

C-INPUT MATERIAL PROPERTIES

OPEN(UNTT=3,FLLE='PHY.DAT, STATUS='OLD')

$\operatorname{READ}(3, *) \mathrm{NDMM}$

$\operatorname{READ}(3, *)(\mathrm{E}(\mathrm{I}), \mathrm{NO}(\mathrm{I}), \mathrm{PO}(\mathrm{I}), \mathrm{I}=1, \mathrm{NDMM})$

CLOSE(3)

C-INPUT EXPLOSION INFORMATION

OPEN(UNIT=4,FILE='EXP.DAT, STATUS='OLD')

$\operatorname{READ}(4, *) \mathrm{NEX}$

READ $\left(4,{ }^{*}\right)(N D E X(I), \operatorname{PNEX}(\mathrm{I}), \operatorname{PSEX}(\mathrm{I}), \mathrm{ANG}(\mathrm{I}), \operatorname{EXTB}(\mathrm{I}), \operatorname{EXTE}(\mathrm{I}), \mathrm{I}=1, \mathrm{NEX})$

CLOSE(4)

DO I=1,NEX

ANG(I)=ANG(I)*3.14159/180.

$\mathrm{KK} 1=\mathrm{NDEX}(\mathrm{I})$

$\operatorname{CO}(K K 1,0)=\operatorname{COS}(A N G(I))$

$\operatorname{SI}(K K 1,0)=\operatorname{SIN}(A N G(I))$

END DO

C-INPUT OTHER INFOMATION

OPEN(UNTT =7,FLE='CAL.DAT',STATUS='OLD')

READ $(7, *) N T, D T, A L F, A M U, C 0, D Y N, B E T A, v i s c o, C C, H H$, iout, delta, bata,

$\%$ koutv

CLOSE(7)

C-_INPUT END HERE

$\mathrm{DT}=\mathrm{DT} * 1 . \mathrm{E}-6$

$\mathrm{AA}=1 .-\mathrm{ALF}^{*} \mathrm{DT} * 0.5$

$\mathrm{BB}=1 .+\mathrm{ALF}^{*} \mathrm{DT} * 0.5$

const $=1.5 *$ visco*3.14159*R(1)**1.5

C-_-SET SOME INITIAL VALUES 
$\mathrm{DO} I=1, \mathrm{ND}$

DO. $\mathrm{J}=1,40$

FNM $(I, J)=-1$.

TFNM $(\mathrm{I}, \mathrm{J})=1$.

END DO

END DO

DO $I=1,1000$

DO L $=1,2$

$\operatorname{accx}(\mathrm{i}, \mathrm{L})=0$.

$\operatorname{accy}(i, L)=0$.

$\operatorname{ACCW}(\mathrm{I}, \mathrm{L})=0$.

DO $J=1,10$

$\operatorname{DELT}(\mathrm{I}, \mathrm{J}, \mathrm{L})=0$

END DO

END DO

END DO

DO $I=1,1000$

DO $\mathrm{J}=0,10$

$\operatorname{NCT}(I, J)=-1$

END DO

END DO

$\mathrm{r}=\mathrm{r}(1)$

C-INITIATE

dneighbo $=r(1) * 4.5$

$\mathrm{K}=\mathbf{0}$

C-_-GET CURRENT EXPLOSIVE FORCE

CALL EXPF(ND,K,NEX,FNW,FSW,PNEX,PSEX,EXTB,EXTE,NDEX,2,NCT)

C_CALCULATE STIFFNESS AND DAMPING FACTOR

CALL PHY(E,PO,NO,KN,M,IZ,R,TH,DTM,DTIZ,BK,BKS,KS)

C-_ OBTAIN CONTACT INFORMATION AND RELATIVE DISTANCE OF

C- DISKS

CALL DGR0(ND,X1,Y1,R)

CALL DGR(ND,X1,Y1,R,SI,CO,DU,NCT,FNW,FSW)

DO I=1,ND

$X(I, 1)=X 1(I)$

$\mathrm{Y}(\mathrm{I}, \mathrm{l})=\mathrm{Y} 1(\mathrm{I})$

END DO

C-_ENTER CALCULATE LOOP

$999 \quad \mathrm{~K}=\mathrm{K}+1$

C___ write $\left(6,{ }^{*}\right) \mathrm{k}$

$\mathrm{KJ}=\mathrm{KJ}+\mathbf{l}$ 


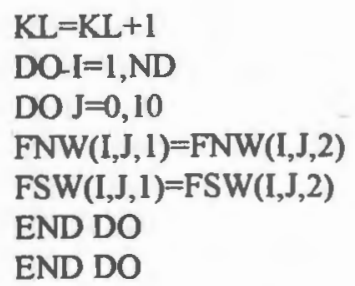

C-C-CALCULATE POSITIONS AND VELOCITIES OF DISKS

CALLVDX(ND,NT,NCT,U,V,W,FNW,FSW,DTM,DTIZ,CO,SI,R,X,Y,BK. BKS,K) DO $\mathrm{I}=1, \mathrm{ND}$

$\mathrm{U}(\mathrm{I}, 1)=\mathrm{U}(\mathrm{I}, 2)$

$\mathrm{V}(\mathrm{I}, \mathrm{1})=\mathrm{V}(\mathrm{I}, 2)$

$\mathrm{W}(\mathrm{I}, 1)=\mathrm{W}(\mathrm{I}, 2)$

$X(I, 1)=X(I, 2)$

$Y(I, 1)=Y(I, 2)$

$\mathrm{Xl}(\mathrm{I})=\mathrm{X}(\mathrm{I}, 2)$

$Y 1(I)=Y(I, 2)$

END DO

C___-OBTAIN NEW CONTACT INFORMATION AND RELATIVE DISTANCE OF DISKS

TIMER=UVMAXF*2.*DT+TIMER

if(timer.gt.dneighbo)then

timer $=0$.

CALL DGR0(ND,X1,Y1,R)

write $(6, *)^{\prime}$ timer $=0$, calculate neighbour disks. $\mathrm{K}=$ ',K

end if

CALL DGR(ND,X1,Y1,R,SI,CO,DIJ,NCT,FNW,FSW)

C--.-GET CURRENT EXPLOSIVE FORCE

CALL EXPF(ND,K,NEX,FNW,FSW,PNEX,PSEX,EXTB,EXTE,NDEX,2,NCT)

C_-_COMPUTE CONTACT FORCE

CALL FORCE(ND,NT,FNW,FSW,NCT,DIJ,KN,KS,U,V,W,CO,SI,R)

C---RECORD MAXTUM FORCE AND STORE FORCE IN FS AND FN

2001 CALL FST(ND,K.FNW,FSW.FNM.TFNM,NG,NCT)

IF(K.LT.NT) GO TO 999

C-LOOP END AND OUTPUT

OPEN(UNIT=9,FILE='outfil'.STATUS='new')

DO L $=1 . N D$

DO $\mathrm{J}=1,40$

IF(FNM(L.J).GT.0.)THEN 


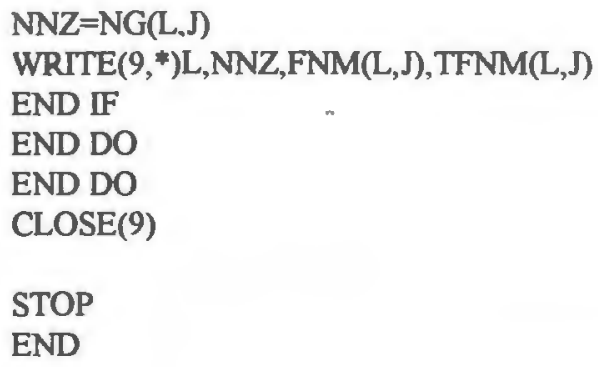

C FOLLOWING 4 LINES SUPPLY GEOMETRIC INFORMATION OF DISK I IG=NDG(I)

C.......CHECK DISK J

DO $170 \mathrm{~J}=1, \mathrm{ND}$

IF(I.EQ.J)GO TO 170

C FOLLOWING 4 LINES GIVE GEOMETRIC INFORMATION OF DISK J

$$
\begin{aligned}
& \mathrm{JG}=\mathrm{NDG}(\mathrm{J}) \\
& \mathrm{RJ}=\mathrm{R}(\mathrm{JG}) \\
& \mathrm{XCJ}=\mathrm{XI}(\mathrm{J})
\end{aligned}
$$


$\mathrm{YCJ}=\mathrm{Y} 1(\mathrm{~J})$

$\mathrm{DD}=\mathbf{0}$.

$\mathrm{XIJ}=\mathrm{XCJ}-\mathrm{XCI}$

$Y \mathrm{JJ}=\mathrm{YCJ}-\mathrm{YCI}$

$\mathrm{DD}=\mathrm{SQRT}(\mathrm{XIJ} * \mathrm{XIJ}+\mathrm{YIJ} * \mathrm{YIJ})$

C.......IN THE FOLLOEWING LINE 2.2 IS A FACTOR TO SET THE RADIUS OF

C NEIGHBORHOOD

$\mathrm{RIJ}=(\mathrm{RI}+\mathrm{RJ}) * 2.2$

IF(DD.LT.RIJ)THEN

NCTO(I,L9) $=$ J

L9 $=$ L9+I

END IF

170 CONTINUE

IF(L9.GT.20) WRITE(6, *)L9>20!-STOP!!!!!!!!!!!!!!!!!!!'

180 CONTINUE

RETURN

END

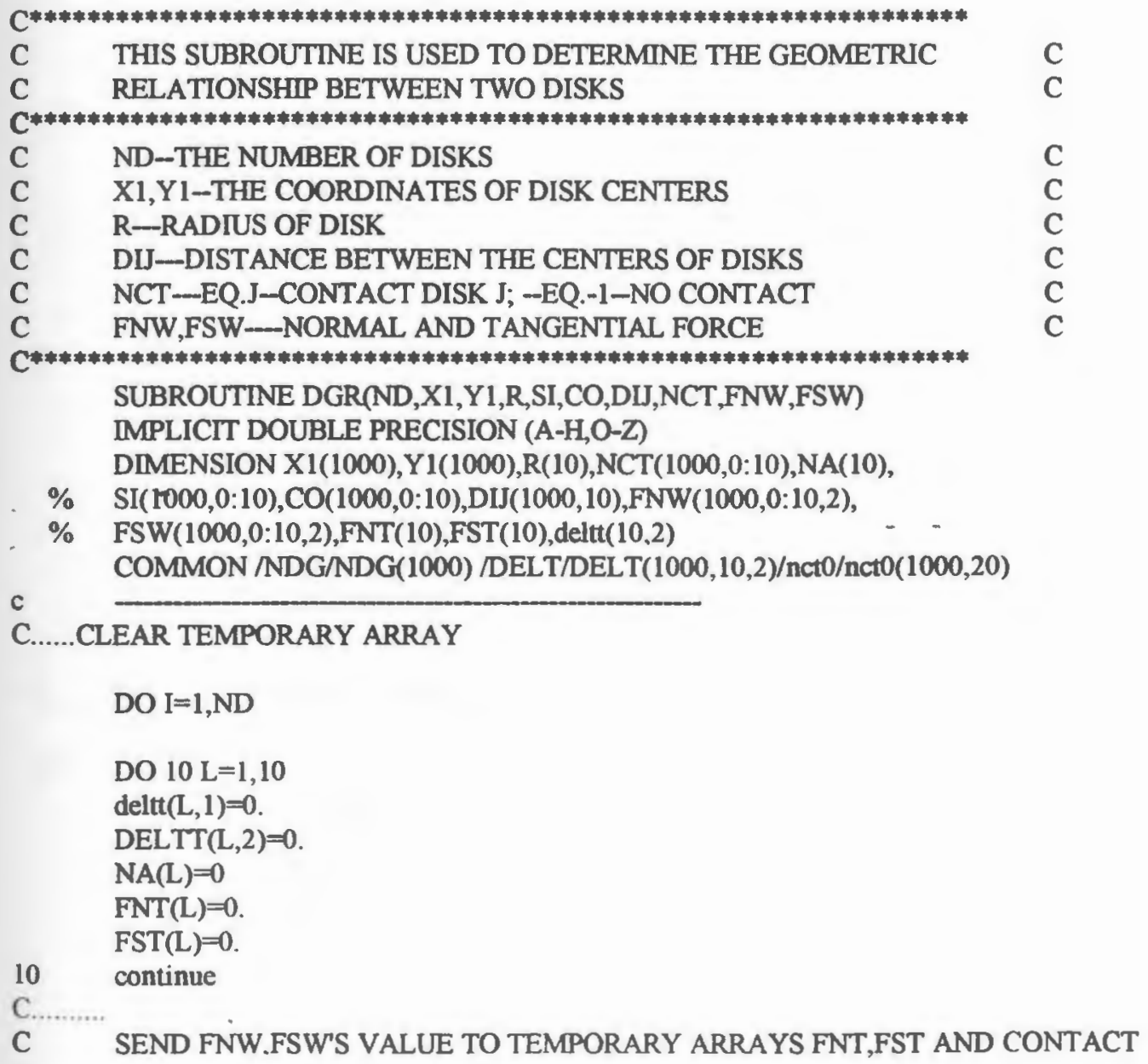


C DISC NUMBER NCT(I,L) TO NA(10)

$\mathrm{DO} \mathrm{L}=1,10$

[F(NCT(I,L).NE.-1) TḦEN

$N A(L)=N C T(I, L)$

$\operatorname{DELTT}(\mathrm{L}, 1)=\operatorname{DELT}(\mathrm{I}, \mathrm{L}, 1)$

DELTT(L, 2) $=\operatorname{DELT}(\mathrm{I}, \mathrm{L}, 2)$

FNT(L) $=$ FNW $(I, L, 1)$

FST(L)=FSW $(\mathrm{I}, \mathrm{L}, \mathrm{l})$

$\operatorname{NCT}(\mathrm{I}, \mathrm{L})=-1$

END IF

END DO

C.........

C.........DETERMINE WHICH DISK CONTACTED DISK I

$3 \quad \mathrm{~L}=1$

$\mathrm{IG}=\mathrm{NDG}(\mathrm{I})$

RIG=R(IG)

do $160 \ln b=1,20$

$j=n c t 0(i, \operatorname{lnb})$

if(j.eq.-1)go to 161

$\mathrm{JG}=\mathrm{NDG}(\mathrm{J})$

$\mathrm{RJG}=\mathrm{R}(\mathrm{JG})$

IF(I.NE.J)THEN

IF(I.GT.J)THEN

DO $30 \mathrm{LI}=1,10$

IF(NCT(J,LD).EQ.D)THEN

$\mathrm{NCT}(\mathrm{I}, \mathrm{L})=\mathrm{J}$

$\mathrm{SI}(\mathrm{I}, \mathrm{L})=-\mathrm{SI}(\mathrm{J}, \mathrm{LI})$

$\mathrm{CO}(\mathrm{I}, \mathrm{L})=-\mathrm{CO}(\mathrm{J}, \mathrm{LI})$

$\operatorname{DIJ}(\mathrm{I}, \mathrm{L})=\mathrm{DIJ}(\mathrm{J}, \mathrm{LI})$

GO TO 60

END IF

30 CONTINUE

GO TO 160

END IF

$\mathrm{DIJ}=\mathrm{SQRT}\left((\mathrm{Xl}(\mathrm{I})-\mathrm{Xl}(\mathrm{J}))^{* *} 2+(\mathrm{Y} 1(\mathrm{I})-\mathrm{Y} 1(\mathrm{~J}))^{* * 2}\right)$

IF(DIJ1.LT.(RIG+RJG)*1.25)THEN

$\mathrm{SI}(\mathrm{I}, \mathrm{L})=(\mathrm{Y} 1(\mathrm{~J})-\mathrm{Y} 1(\mathrm{I})) / \mathrm{DIJ} 1$

$\mathrm{CO}(\mathrm{I}, \mathrm{L})=(\mathrm{Xl}(\mathrm{J})-\mathrm{X} 1(\mathrm{I})) / \mathrm{DIJ} 1$

$\operatorname{DIJ}(\mathrm{I}, \mathrm{L})=\mathrm{DIJ}$ l-rig-rig

$\operatorname{NCT}(\mathrm{L}, \mathrm{L})=\mathrm{J}$

C.......RETURN ORIGINAL FNW,FSW'S VALUE TO PROPER UNITS OF FNW AND FSW

60 DO $\mathrm{K}=1,10$

IF(NA(K).EQ.J)THEN

FNW $(I, L, 1)=F N T(K)$

FSW(I,L, 1)=FST(K)

$\operatorname{DELT}(\mathrm{I}, \mathrm{L}, 1)=\operatorname{DELTT}(\mathrm{K}, 1)$

$\operatorname{DELT}(\mathrm{I}, \mathrm{L}, 2)=\mathrm{DELTT}(\mathrm{K} .2)$

GO TO 1

END IF

END DO 
FNW(1,L,1)=0.

$\mathrm{FSW}(\mathrm{I}, \mathrm{L}, 1)=0$.

$\operatorname{DELT}(\mathrm{L}, \mathrm{L}, \mathrm{l})=0$.

$\operatorname{DELT}(I, L, 2)=0$.

$\mathrm{L}=\mathrm{L}+1$

END IF

END IF

END DO

161 IF(L.GT.10) WRITE(6,*)L>10!-STOP!!!!!!!!!!!!!!!!!!!"

end do

RETURN

END

\begin{tabular}{|c|c|}
\hline & 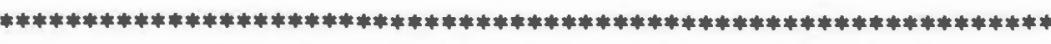 \\
\hline C & THIS SUBROUTINE IS USED TO CALCULATE VELOCITY AND \\
\hline C & DISPLACEMENT OF DISKS \\
\hline$C^{*}$ & 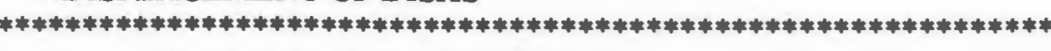 \\
\hline C & ND__-TOTAL NUMBER OF DISKS \\
\hline C & NT-_-_-TOTAL TIME STEPS \\
\hline C & NCT(I,L) EQ.J DISK J IS THE L-th DISK IN CONTACT WITH DISK I \\
\hline C & U,V-VELOCITIES \\
\hline C & W-_ANGULAR VELOCITY \\
\hline C & $\mathrm{X}, \mathrm{Y}$-_-POSITIONS OF DISK CENTER \\
\hline $\mathbf{C}$ & BK.BKS-DAMPING FACTORS \\
\hline $\mathbf{C}$ & K-CURRENT TIME STEPS \\
\hline C & FNW,FSW-CONTACT FORCE \\
\hline C & CO,SI--COSINE,SINE \\
\hline $\mathbf{C}$ & R-RADIUS \\
\hline
\end{tabular}

C*********************FILE NAME IS VDX.FOR**************************

$\mathrm{C}$

C

$\mathrm{C}$

C

C

C

$\mathrm{C}$

C

C

C

C

C

C

SUBROUTINE VDX(ND,NT,NCT,U,V,W,FNW,FSW,DTM,DTIZ,CO,SI,R,X,

$\% \quad \mathrm{Y}, \mathrm{BK}, \mathrm{BKS}, \mathrm{K}$ )

IMPLICIT DOUBLE PRECISION (A-H,O-Z)

DIMENSION U(1000,2), V(1000,2),W(1000,2),

$\% \quad \operatorname{FNW}(1000,0: 10,2), \operatorname{FSW}(1000,0: 10,2), \operatorname{DTM}(10,10), \operatorname{DTIZ}(10,10)$,

$\% \quad \mathrm{CO}(1000,0: 10), \operatorname{SI}(1000,0: 10), \mathrm{R}(10), \mathrm{X}(1000,2), \mathrm{Y}(1000,2)$,

$\% \quad \operatorname{NCT}(1000,0: 10), \mathrm{BK}(10,10), \mathrm{BKS}(10,10)$

COMMON /DT/DT /TH/TH(10) /NDG/NDG(1000)

$\% \quad$ NDM/NDM(1000)/ACC/ACCX(1000,2),ACCY $(1000,2), \operatorname{ACCW}(1000,2)$ common /uvmax/uvmaxf/db/delta, bata

C

C........VELOCITY

C (DX/DT), N+1 $=2 *(\mathrm{D} 2 \mathrm{X} / \mathrm{DT} 2), \mathrm{N}+(\mathrm{DX} / \mathrm{DT}), \mathrm{N}-1$

C $\quad \mathrm{X}, \mathrm{N}+1=2 * \mathrm{X}, \mathrm{N}-\mathrm{X}, \mathrm{N}-1+\mathrm{D} 2 \mathrm{X} / \mathrm{DT} 2, \mathrm{~N} * \mathrm{DT} 2$

$1 \quad$ DO I $=1, \mathrm{ND}$

$\mathrm{U}(1,2)=\mathrm{U}(\mathrm{I}, 1)$

$\mathrm{V}(\mathrm{I}, 2)=\mathrm{V}(\mathrm{I}, 1)$

$\mathrm{W}(\mathrm{I}, 2)=\mathrm{W}(\mathrm{I}, 1)$

$X(I, 2)=X(I, 1)$

$\mathrm{Y}(\mathrm{I}, 2)=\mathrm{Y}(\mathrm{I}, 1)$

END DO

$\mathrm{DO} \mathrm{I}=1, \mathrm{ND}$ 
$\operatorname{ACCX}(1,2)=0$.

$\operatorname{ACCY}(I, 2)=0$.

$\operatorname{ACCW}(1,2)=0$.

C......... IN THE FOLLOWING 5 LINES GEOMETRIC AND MATERIAL INFORMATIONS ARE

C......... GIVEN

$\mathrm{IM}=\mathrm{NDM}(\mathrm{I})$

$\mathrm{IG}=\mathrm{NDG}(\mathrm{I})$

$\mathrm{DTM}=\mathrm{DTM}(\mathrm{IM}, \mathrm{IG})$

DTIZI=DTIZ(IM,IG)

RIG=R(IG)

DO $33 \mathrm{~L}=0,10$

C.......L $=0$ MEANS THE INPUT FORCE

IF(NCT(I,L).GT.-I)THEN

$\mathrm{J}=\mathrm{NCT}(\mathrm{I}, \mathrm{L})$

C..... VELOCITY INCREMENT DUE TO CONTACT FORCE AND INPUT FORCE $\operatorname{ACCX}(1,2)=A C C X(1,2)-D T M{ }^{*}(F N W(1, L, 1) * C O(I, L)+F S W(1, L, 1)$

$\% \quad * \operatorname{SI}(\mathrm{I}, \mathrm{L}))$

ACCY(I, 2)=ACCY(I,2)-DTMI*(FNW(I,L,1)*SI(I,L)-FSW(I,L,I)

$\left.\% \quad{ }^{*} \mathrm{CO}(1, L)\right)$

$\operatorname{ACCW}(\mathrm{I}, 2)=\mathrm{ACCW}(\mathrm{I}, 2)+\mathrm{FSW}(\mathrm{I}, \mathrm{L}, \mathrm{1}) * \mathrm{RIG} * \mathrm{DTTZI}$

C..........VELOCITY INCREMENT DUE TO DAMPING FORCE

IF(J.GT.0)THEN

$\mathrm{JM}=\mathrm{NDM}(\mathrm{J})$

$\mathrm{JG}=\mathrm{NDG}(\mathrm{J})$

THM=MIN(TH(IG),TH(JG))

$\mathrm{Z}=(\mathrm{U}(\mathrm{I}, \mathrm{I})-\mathrm{U}(\mathrm{J}, \mathrm{I}))^{*} \mathrm{CO}(\mathrm{I}, \mathrm{L})+(\mathrm{V}(\mathrm{I}, \mathrm{I})-\mathrm{V}(\mathrm{J}, \mathrm{I}))^{*} \mathrm{SI}(\mathrm{I}, \mathrm{L})$

$Z A=Z * B K(I M, J M) * T H M$

$\mathrm{ZS}=(\mathrm{V}(\mathrm{I}, 1)-\mathrm{V}(\mathrm{J}, \mathrm{1}))^{*} \mathrm{CO}(\mathrm{I}, \mathrm{L})-(\mathrm{U}(\mathrm{I}, \mathrm{l})-\mathrm{U}(\mathrm{J}, \mathrm{1})) * \mathrm{SI}(\mathrm{I}, \mathrm{L})$

$\%+(\mathrm{W}(\mathrm{I}, 1) * \mathrm{R}(\mathrm{IG})+\mathrm{W}(\mathrm{J}, 1) * \mathrm{R}(\mathrm{JG}))$

$Z A S=Z S^{*} B K S(\mathbb{M}, J M) * T H M$

$\operatorname{ACCX}(1,2)=\operatorname{ACCX}(1,2)-(Z A * C O(I, L)-Z A S * S I(I, L)) * D T M I$

$\operatorname{ACCY}(\mathrm{I}, 2)=\operatorname{ACCY}(\mathrm{I}, 2)-\left(\mathrm{ZA} * \mathrm{SI}(\mathrm{I}, \mathrm{L})+\mathrm{ZAS}{ }^{*} \mathrm{CO}(\mathrm{I}, \mathrm{L})\right) * \mathrm{DTMI}$

$\operatorname{ACCW}(\mathrm{I}, 2)=A C C W(I, 2)-Z A S * R(I G) * D T I Z I$

END IF

END IF

33 CONTINUE

$\mathrm{U}(1,2)=\mathrm{U}(\mathrm{I}, 1)+(\mathrm{ACCX}(\mathrm{I}, 1) *(1$.delta)+delta*ACCX $(\mathrm{I}, 2))$

$\mathrm{V}(\mathrm{I}, 2)=\mathrm{V}(\mathrm{I}, 1)+(\operatorname{ACCY}(\mathrm{I}, 1) *(1$-delta)+delta $* \operatorname{ACCY}(\mathrm{I}, 2))$

$W(I, 2)=W(I, 1)+(\operatorname{ACCW}(I, 1) *(1$. -delta $)+$ delta*ACCW $(I, 2))$

$\mathrm{X}(\mathrm{I}, 2)=\mathrm{X}(\mathrm{I}, 1)+\mathrm{U}(\mathrm{I}, 1) * \mathrm{DT}+((0.5$-bata $) * \mathrm{ACCX}(\mathrm{I}, 1)$

$\% \quad+$ bata*ACCX(I,2) *DT

$\mathrm{Y}(\mathrm{I}, 2)=\mathrm{Y}(\mathrm{I}, 1)+\mathrm{V}(\mathrm{I}, 1) * \mathrm{DT}+\left((0.5 \text {-bata })^{*} \mathrm{ACCY}(\mathrm{I}, 1)\right.$

$\% \quad$ +bata*ACCY $(\mathrm{I}, 2))^{*} \mathrm{DT}$

C..........NEW COORDINATE OF THE CENTERS

$\mathrm{absu}=\mathrm{abs}(\mathrm{u}(\mathrm{i}, 2))$

$a b s v=a b s(v(i, 2))$

uvmax=dmax l(absu,absv) 
uvmaxf=dmax l (uvmax,uvmaxf)

$$
\begin{aligned}
& \operatorname{ACCX}(I, 1)=A C C X(I, 2) \\
& \operatorname{ACCY}(I, 1)=A C C Y(I, 2) \\
& \operatorname{ACCW}(I, 1)=A C C W(I, 2) \\
& \text { END DO }
\end{aligned}
$$

\begin{tabular}{|c|c|c|}
\hline \multicolumn{3}{|c|}{ 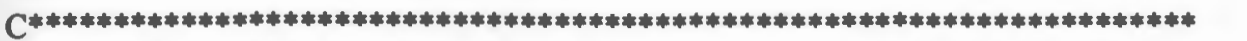 } \\
\hline C & THIS SUBROUTINE IS USED TO CALCULATE EXPLOSIVE FORCE & \\
\hline \multicolumn{3}{|c|}{ 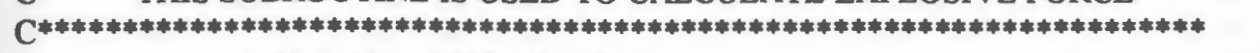 } \\
\hline C & ND-TOTAL NUMBER OF DISKS & \\
\hline C & K-CURRENT TLME STEP & C \\
\hline C & NDEX-THE NUMBER OF DISK ON WHICH THERE IS AN EXPLOSION & \\
\hline C & PNEX--NORMAL FORCE OF EXPLOSION & \\
\hline C & PSEX-TANGENTIAL FORCE OF EXPLOSION & \\
\hline C & FNW--NORMAL FORCE (WORK UNIT) & \\
\hline $\mathrm{C}$ & FSW-TANGENTIAL FORCE (WORK UNIT) & \\
\hline C & EXTB-TIME EXPLOSION BEGINS & \\
\hline C & EXTE-TIME EXPLOSION ENDS & \\
\hline C & NEX - THE TOTAL NUMBER OF EXPLOSIVE FORCES & \\
\hline $\mathbf{C}$ & DT_-TIME STEP & \\
\hline C & NCT-_USED TO SHOW DISKS CONTACT & \\
\hline
\end{tabular}

RETURN

END

SUBROUTINE EXPF(ND,K,NEX,FNW,FSW,PNEX,PSEX,EXTB,EXTE,NDEX,KW,NCT) IMPLICIT DOUBLE PRECISION (A-H,O-Z) DIMENSION FNW(1000,0:10,2),FSW(1000,0:10,2),PNEX(20),PSEX(20),

$\% \quad \operatorname{EXTB}(20), \operatorname{EXTE}(20), \operatorname{NDEX}(20), \operatorname{NCT}(1000,0: 10)$ COMMON /DT/DT

C

C......THE EXPLOSIVE LOADINGS ARE ASSUMED AS TRIANGULAR TIME DEPENDENTS

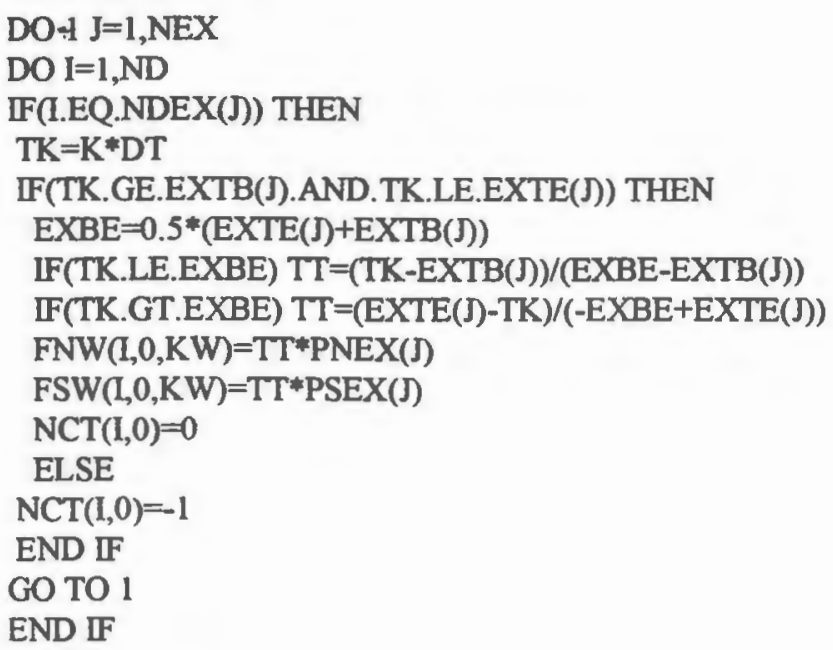




$1 \quad \begin{array}{ll}\text { END DO } \\ \text { CONTINUE } \\ \text { RETURN } \\ \text { END }\end{array}$

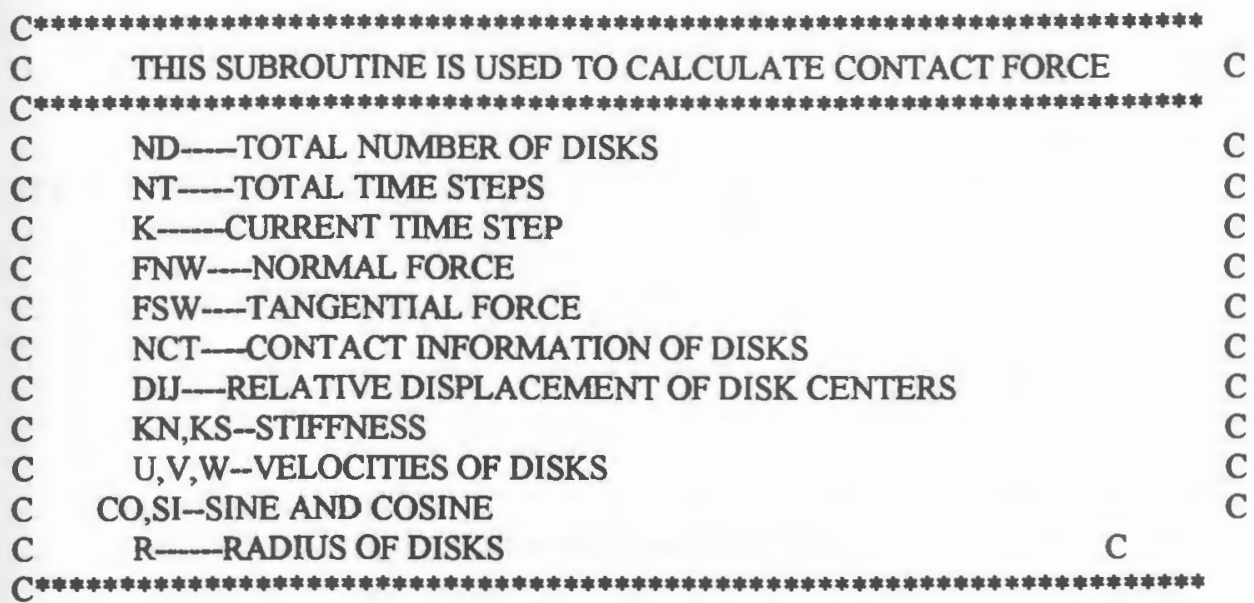

SUBROUTINE FORCE(ND,NT,FNW,FSW,NCT,DIJ,KN,KS,U,V,W,CO,SI,R)

IMPLICIT DOUBLE PRECISION (A-H, O-Z)

REAL KN(10,10),KS(10,10)

DIMENSION FNW(1000,0:10,2),FSW(1000,0:10,2),NCT(1000,0:10),

$\% \quad \operatorname{CO}(1000,0: 10), \operatorname{SI}(1000,0: 10), \mathrm{DIJ}(1000,10), \mathrm{U}(1000,2), \mathrm{V}(1000,2)$,

$\% \quad W(1000,2), R(10)$

COMMON IDT/DT /AMU/AMU /CO/C0 /NDG/NDG(1000)/NDM/NDM(1000)

$\% \pi /$ TH/TH(10)/const/const, CC,visco,HH/DELT/DELT $(1000,10,2)$

DO $20 \mathrm{I}=1, \mathrm{ND}$

c obtain geometric and material information of particle $i$

$\mathrm{IM}=\mathrm{NDM}(\mathrm{I})$

IG $=$ NDG(I)

$\mathrm{RIG}=\mathrm{R}(\mathrm{IG})$

c search particles contacting with particle i

DO $10 \mathrm{~L}=1,10$

IF(NCT(I,L).GT.0) THEN

C found $j$ contacting $i$

$\mathrm{J}=\mathrm{NCT}(\mathrm{I}, \mathrm{L})$

c obtain geometric and material information of particle $j$

$\mathrm{JM}=\mathrm{NDM}(\mathrm{J})$

$\mathrm{JG}=\mathrm{NDG}(\mathrm{J})$

$\mathrm{RJG}=\mathrm{R}(\mathrm{JG})$

THMIN=MIN(TH(IG),TH(JG))

c ddd is the distance between $\mathrm{i}$ and $\mathrm{j}$ if consider them as rigid ddd $=\operatorname{dij}(i, 1)$ 
vvvl is the normal relative velocity betw' $\mathrm{i}$ and $\mathrm{j}$

$\mathrm{vwvl}=(\mathrm{U}(\mathrm{I}, 2)-\mathrm{U}(\mathrm{J}, 2)) * \mathrm{CO}(\mathrm{I}, \mathrm{L})+(\mathrm{V}(\mathrm{I}, 2)-\mathrm{V}(\mathrm{J}, 2)) * \mathrm{SI}(\mathrm{L}, \mathrm{L})$

IF(VVV1.LT.0.)THEN

$\operatorname{delt}(\mathbf{i}, 1,2)=\operatorname{del}(\mathbf{i}, 1,2)+\mathrm{vvv} 1 * \mathrm{dt}$

if(DELT( $1, L, 2) . L E .0$.$) then$

finw(i,1,2)=0.

$\operatorname{DELT}(\mathrm{I}, \mathrm{L}, 2)=0$.

else

$111 \quad$ Fnw $(i, 1,2)=\propto c * \operatorname{delt}(i, 1,2) * * 1.5$

end if

hofdisks=ddd+delt $(\mathrm{i}, 1,2)$

go to 22

END IF

$\mathrm{cl}=\mathrm{THMIN} *$ CONST

dddd =sqrt(ddd*ddd $\left.+4 . *(\mathrm{cl} / \mathrm{cc} * \mathrm{vvv} 1)^{* *} 0.66666667\right)$

$\operatorname{delt}(\mathrm{i}, 1,2)=0.5 *$ (-ddd +dddd)

$11 \quad \mathrm{FNW}(\mathrm{I}, \mathrm{L}, 2)=\mathrm{CC} * \operatorname{DELT}(\mathrm{I}, \mathrm{L}, 2) * * 1.5$

hofdisks $=0.5 *$ (ddd+dddd)

$22 \mathrm{~min}=\min (\mathrm{rig}, \mathrm{rjg})$

if (hofdisks.lt.1.e-11) then

hofdisks=1.e-11

end if

hdifoverr $=$ (hh-hofdisks)/rmin

c if(hofdisks.1t.0.)then

c write(6,*)'solid contact',vvvl,ddd, dddd, delt(i,1,2), $i_{2}$

c end if

c calculate tangential force

if(hdifoverr.ge. 0 .) then

$x x x=\min *$ sqrt(hdifoverr*(1.-hdifover $\left.{ }^{*} 0.25\right)$ )

$\mathrm{dv}=((\mathrm{V}(\mathrm{I}, 2)-\mathrm{V}(\mathrm{J}, 2)) * \mathrm{CO}(\mathrm{I}, \mathrm{L})$

$\% \quad-(U(I, 2)-U(J, 2)) * S I(I, L)+(W(I, 2) * R I G+W(J, 2) * R(J G)))$

FSW(I,L, 2) $=$ thmin *dv*(xxx/abs(hofdisks) $)^{*}$ visco

if(abs(fsw(i,1,2)).gt.2.)write(77,*)' $i={ }^{\prime}, 1,{ }^{\prime} j=$ ',j,

$\%$ 'dv= ',dv,' xxx= ',uxx.' hofdisks = ', hofdisks,' vvvl =',vvv

else

fsw $(i, 1,2)=0$.

end if

ELSE

FNW $(1, L, 2)=0$.

FSW $(I, L, 2)=0$.

END IF

10 CONTINUE

20 CONTINUE

RETURN

END 


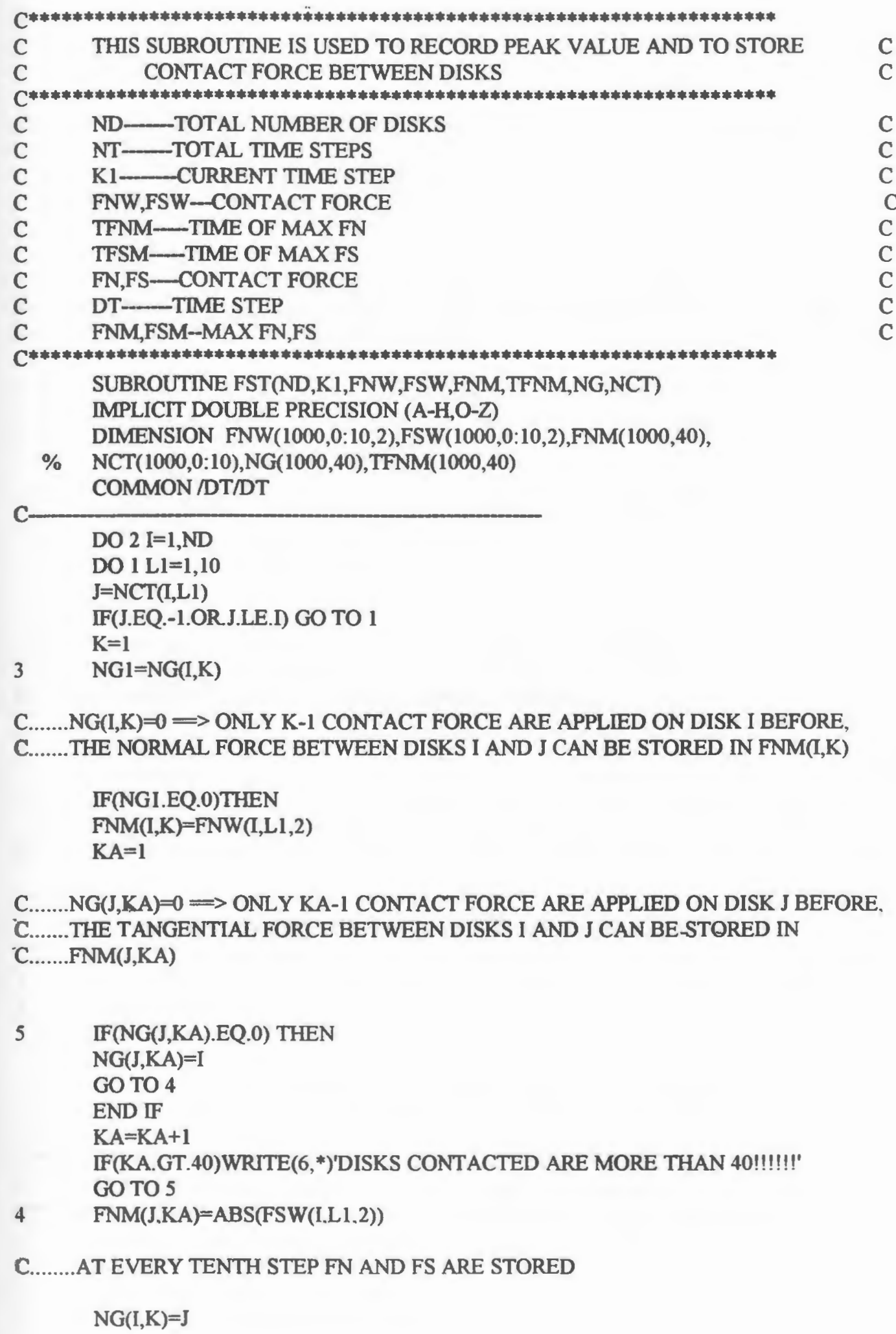


GO TO 1

ELSE

C.......IS FNM(I,K) THE NORMAL FORCE BETWEEN DISK I AND DISK J?

IF(J.EQ.NG1)THEN

C....... YES, STORE THE LARGER ONE AND THE TIME.

IF(FNM(I,K).LT.FNW(I,L1,2))THEN

FNM(I,K)=FNW(I,L1,2)

$\operatorname{TFNM}(\mathrm{I}, \mathrm{K})=\mathrm{K} 1 * \mathrm{DT}$

END IF

C.......FIND THE ELEMENT STORED THE TANGENTIAL FORCE, AND COMPARE WITH C.......CURRENT VALUE, STORE THE LARGER.

$A B W=A B S(F S W(I, L 1,2))$

$\mathrm{KA}=1$

7 IF(NG(J,KA).EQ.I) THEN

IF(FNM(J,KA).LT.ABW) THEN

$F N M(J, K A)=A B W$

TFNM $(J, K A)=K 1 * D T$

END IF

GO TO 6

END IF

$\mathrm{KA}=\mathrm{KA}+1$

GO TO 7

C.......AT EVERY TENTH STEP FN AND FS ARE STORED

$6 \quad$ GO TO 1

END IF

END IF

C.......NO, CHECK THE NEXT

$\mathrm{K}=\mathrm{K}+1$

IF(K.GT.40)WRITE(6,*)'DISKS CONTACTED MORE THAN 40!' -

GO TO 3

1 CONTINUE

2 CONTINUE

RETURN

END

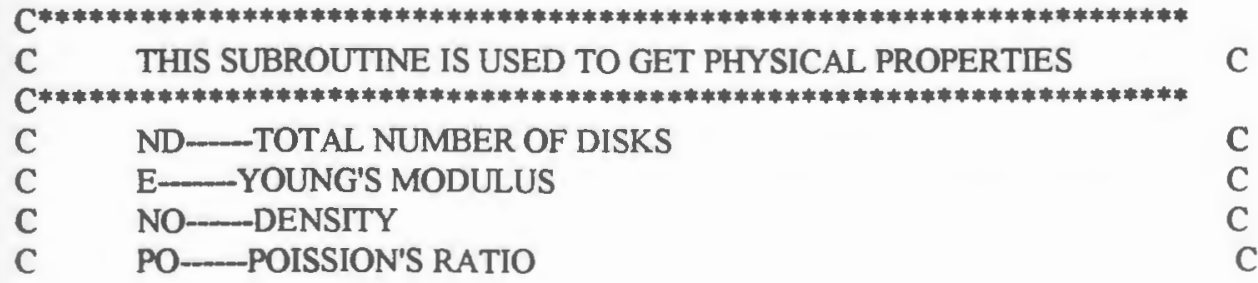




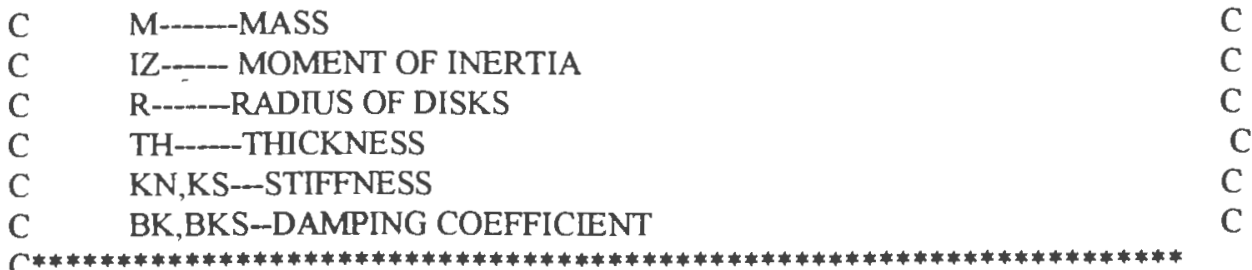

C

SUBROUTINE PHY(E,PO,NO,KN,M,IZ,R,TH,DTM,DTIZ,BK,BKS,

$\% \quad \mathrm{KS})$

IMPLICIT DOUBLE PRECISION (A-H,O-Z)

REAL KN(10,10),M(10,10), NO(10), IZ(10,10),KS(10,10)

DIMENSION E(10),PO(10), TH(10),R(10),DTM(10,10),DTIZ(10,10),

$\% \quad \operatorname{BK}(10,10), \operatorname{BKS}(10,10)$

COMMON /DT/DT /DYN/DYN/BETA/BETA /NDGM/NDGM/NDMM/NDMM

C

c WRITE $\left(6,{ }^{*}\right)^{\prime}$ WANT TO CONSIDER TANGENTIAL FORCE? '

c WRITE $\left(6,{ }^{*}\right)^{\prime} \mathrm{KS}=\mathrm{A} * \mathrm{KN}$, INPUT VALUE OF A *

c $\quad \operatorname{READ}\left(5,{ }^{*}\right) \mathrm{SK}$

$\mathrm{sk}=0$.

C......PLANE STRESS STATE IS ASSUMED.

DO I $=1, \mathrm{NDMM}$

$\mathrm{E}(\mathrm{I})=\mathrm{E}(\mathrm{I})^{*}(1 .+2 . * \mathrm{PO}(\mathrm{I})) /(1 .+\mathrm{PO}(\mathrm{I}))^{* * 2}$

$\mathrm{PO}(\mathrm{I})=\mathrm{PO}(\mathrm{I}) /(\mathrm{l}+\mathrm{PO}(\mathrm{I}))$

end do

do $\mathrm{i}=1, \mathrm{ndmm}$

DO J $=1$, NDGM

$\mathrm{M}(\mathrm{I}, \mathrm{J})=\mathrm{NO}(\mathrm{I}) * 3.14159 * \mathrm{R}(\mathrm{J}) * * 2 * \mathrm{TH}(\mathrm{J})$

$\mathrm{IZ}(\mathrm{I}, \mathrm{J})=0.5 * \mathrm{M}(\mathrm{I}, \mathrm{J}) * \mathrm{R}(\mathrm{J}) * 2$

$\mathrm{DTM}(\mathrm{I}, \mathrm{J})=\mathrm{DT} / \mathrm{M}(\mathrm{I}, \mathrm{J})$

$\mathrm{DTIZ}(\mathrm{I}, \mathrm{J})=\mathrm{DT} / \mathrm{IZ}(\mathrm{I}, \mathrm{J})$

END DO

C......STIFFNESS AND DAMPING FACTOR

DO $J=1, N D M M$

$\mathrm{EP}=\left(1 .-\mathrm{PO}(\mathrm{I})^{* *} 2\right) / \mathrm{E}(\mathrm{I})+\left(1 .-\mathrm{PO}(J)^{* *} 2\right) / \mathrm{E}(\mathrm{J})$

$K N(I, J)=3.1415927 /\left(7.5^{*} \mathrm{EP}\right)$

$\mathrm{KS}(\mathrm{I}, \mathrm{J})=\mathrm{KN}(\mathrm{I}, \mathrm{J}) * \mathrm{SK}$

$\mathrm{BK}(\mathrm{I}, \mathrm{J})=\mathrm{BETA} * \mathrm{KN}(\mathrm{I}, \mathrm{J})$

$\operatorname{BKS}(\mathrm{I}, J)=\mathrm{BETA} * \mathrm{KS}(\mathrm{I}, \mathrm{J})$

END DO

END DO

RETURN

END 


\section{BIBLIOGRAPHY}

Bagster, D. F. and Kirk, R. (1985) "Computer generation of a model to simulate granular material behavior", Journal of Powder \& Bulk Solids Technology, vol. 9, pp. 19-24.

Barbosa, R. E. and Ghaboussi, J. (1990) "Discrete finite element method for multiple deformable bodies", Finite Elements in Analysis and Design, vol. 7, pp. 145-158.

Bazant, Z. P., Krizek, R. J. and Shieh, C. L. (1983) "Hysteretic endochronic theory for sand", Journal of Engineering Mechanics, vol. 109, pp. 1073-1095.

Biot, M. A. (1956a) "The theory of propagation of elastic waves in a fluidsaturated porous solid, I lower frequency range", Journal of Acoustical Society of America, vol. 28, pp. 168-178.

Biot, M. A. (1956b) "Theory of propagation of elastic waves in a fluid-saturated porous solid, II higher frequency range", Journal of Acoustical Society of America, vol. 28, pp. 179-191.

Biot, M. A. (1962) "Mechanics of deformation and acoustic propagation in porous media", Journal of Applied Physics, vol. 23, pp. 1482-1498.

Bourbie, T., Coussy, O. and Zinszner, B. (1987) Acoustics of Porous Media, Gulf Publishing Company, Texas.

Brandt, H. (1955) "A study of the speed of sound in porous granular media", Journal of Applied Mechanics, vol. 22.

Brown, J.W., Murnell, D. W. and Stout, J. H. (1980) "Propagation of explosive shock waves through rubble screens", Misc. Paper SL-80-7, U.S. Army Engineer Waterways Experiment Station, Vickburg, MS.

Cameron, A. (1966) The Principles of Lubrication, Longman, London.

Carroll, M. M. and Holt, A. C. (1972) "Static and dynamic pore-collapse relation for ductile porous materials", Journal of Applied Physics, vol. 43, pp. 1626-1636.

Christiansen, H. (1970) "Ehd theory of spherical bodies in normal approach", Transaction of American Society of Mechanical Engineers, Journal of Lubrication Technology, vol. 92, pp. 145-154. 
Cundall, P. A. and Strack, O. D. L. (1979) "A discrete numerical model for granular assemblies", Geotechnique, vol. 29, no. 1, pp. 47-65.

Cundall, P. A., Marti, J, Beresford, P., Last, N. and Asgian, M. (1978) "Computer modeling of jointed rock masses", Technical Report N-78-4, U.S. Army Engineering Waterways Experiment Station, Vicksburg, MS.

Drescher, A. and De Josselin De Jong, G. (1972) "Photoelasticity verification of a mechanical model for the flow of granular materials", Proceedings of IUTAMSymposium on Optical Methods in Solid Mechanics, Poitiers, France.

Drescher, A. (1979) "Application of photoelasticity to investigation of constitutive laws for granular materials", Proceedings of IUTAM-Symposium on optical methods in Solid Mechanics, Poitiers, France.

Durelli, A. J. and Wu, D. (1983) "Use of coefficients of influence to solve some inverse problems in plane elasticity", Journal of Applied Mechanics, vol. 50, pp. 288-296.

Duffy, J. and Mindlin, R. D. (1957) "Stress-strain relations and vibration of a granular medium", Journal of Applied Mechanics, vol. 24, pp. 585-593.

Endley, S. N. and Peyrot, A. H. (1977) "Load distribution in granular media", Joumal of the Engineering Mechanics Division, ASCE, vol.103, pp. 99-111.

Gassman, F. (1951) "Elastic waves through a packing of spheres", Geophysics, vol. 16, pp. 673-685.

Gohar, R. (1988) Elastohydrodynamics, Ellis Horwood Limited, England.

Goodman, M. A. and Cowin, S. C. (1972) "A continuum theory for granular materials", Archive of Rational Mechanics and Analysis, vol. 44.

Hudson, J. A. (1968) "The scattering of elastic waves by granular media", Journal of Applied Mechanics and Applied Mathematics, vol. 21, pp. 487-508.

Hughes, D. S. and Cross, J. H. (1951) "Elastic wave velocities in rocks at high pressures and temperatures", Geophysics, vol. 14, no. 4, pp. 577-592.

Hughes, D. S. and Kelly, J. L. (1952) "Variation of elastic wave velocity with saturation in sandstone", Geophysics, vol. 17, pp. 739-752.

Iida, K. (1939) "The velocity of elastic waves in sand", Bulletin Earthquake Research Institute, Japan, vol. 17. 
Jones, T. and Nur, A. (1983) "Velocity and attenuation in sandstones at elevated temperatures and pressures", Geoph. Res. Lett, vol. 10, pp. 140-143.

Johnson, K. L. (1985) Contact Mechanics, Cambridge University Press.

Kishino, Y. (1988) "Disc model analysis of granular media", in Micromechanics of Granular Materials, Ed. by M. Satake and J. T. Jenkins, Elsevier Science Publishers B. V., Amsterdam, pp. 143-152.

Lee, K. M. and Cheng, H. (1973) "The pressure and deformation profiles between two normally approaching lubricated cylinders", Transactions of American Society of Mechanical Engineers, Journal of Lubrication Technology, (paper No. 72-Lub38).

Mavko, G. M. and Nur, A. (1979) "Wave attenuation in partially saturated rocks", Geophysics, vol. 44, pp. 161-178.

Nelson, R. B., Lade, P. V., Issa, J., Chamieh, N. and Yamamuro, J. (1988) "Micromechanical Behavior of Frictional Geologic Materials", Final Report to AFOSR under contract number AFOSR-86-0290, Bolling AFB, Washington, D. C., pp. 1-26.

Nemat-Nasser, S. (1982) "Fabric and its influence on mechanical behavior of granular materials", in Deformation and Failure of Granular Materials, ed., Vermeer, P. A. and Lugar, H. J., Balkema, A. A., pp. 77-94.

Nunziato, J. and Walsh, E. (1977) "on the influences of void compaction and material non-uniformity on the propagation of one-dimensional acceleration waves in granular materials", Archive for Rational Mechanics and Analysis, vol. 64, pp. 299-316.

Oda, M. (1972) "Initial fabrics and their relations to mechanical properties of granular material", Soils and Foundations, vol. 12, no. 1, pp. 17-36.

Ogushwitz, P. R. (1985) "Applicability of the Biot Theory, I. Low-Porosity Materials; II. Suspensions; III. Waves Speeds Versus Depth in Marine Sediments", Journal of Acoustic Society of America, vol. 77, pp. 429.

Palmer, I. D. and Traviolia, M. L. (1981) "Attenuation by squirt flow in undersaturated gas sands", Geophysics, vol. 45, pp. 1780-1792.

Petrakis, E. and Dobry, R. (1988) "A two-dimensional numerical micromechanical model for a granular cohesionless materials at small strains" Proceedings of American Physical Society, New Orleans, LA. 
Pan, X. D. and Reed, M. B. (1991) "A coupled distinct-finite element method for large deformation analysis of rock masses", International Journal of Rock Mechanics and Mining Science and Geomechanics Abstract, vol. 28, no. 1, pp. 9399.

Plona, T. J. (1982) "Acoustics of fluid-saturated porous media", Ultrasonics symposium, IEEE, vol. 2, pp. 1044-1048.

Powell, M. J. (1980) "Computer-simulated random packing of spheres", $\underline{\text { Powder }}$ Technology, vol. 25, pp. 45.

Sadd, M. H. and Hossain, M. (1988) "Wave propagation in distributed bodies with applications to dynamic soil behavior", Journal of Wave-Material interaction, vol. 3, pp. 311-326.

Sadd, M. H., Shukla, A. and Mei, H. (1989a) "Computational and experimental modeling of wave propagation in granular materials", Proceedings of the 4th International Conference on Computational Methods and Experimental Measurements, pp. 325-334, Capri, Italy.

Sadd, M. H., Shukla, A., Mei, H. and Zhu, C. Y. (1989b) "The effect of voids and inclusions on wave propagation in granular materials", in Micromechanics and Inhomogeneity - The Toshio Mura Anniversary Volume, ed. Weng, G. L., Taya, M. and Abe, H., Springer-Verlag, New York.

Sadd, M. H., Qiu, L., Boardman, W. and Shukla, A. (1991) "Wave propagation in granular media using cohesionless elastic networks", International Journal of Rock Mechanics and Mining Science and Geomechanics Abstracts", vol. 29, pp. 161170.

Sadd, M. H., Tai, Q. M. and Shukla, A. (1993) "Contact law effects on wave propagation in particulate materials using distinct element modeling", International Journal of Nonlinear Mechanics, vol. 28, pp. 251-265.

Satake, M. and Jenkins, J. T. (1982) Mechanics of granular materials-new models and constitutive relations, Proceedings of U.S./Japan Seminar on New Models and Constitutive Relations in the Mechanics of Granular Materials, Elsevier, Cornell University.

Shukla, A. and Rossamanith, H. P. (1986) "Dynamic photoelastic investigation of wave propagation and energy transfer across contacts", Journal of Strain Analysis, vol. 21 , no. 4, pp. 213-218. 
Shukla, A. and Damania, C. (1987) "Experimental investigation of wave velocity and dynamic contact stress in an assembly of disks", Journal of Experimental Mechanics, vol. 27, no. 3, pp. 268-281.

Shukla, A., Zhu, C. Y. and Sadd, M. H. (1988a) "Angular dependence of dynamic load transfer due to explosive loading in two dimensional granular aggregates", Journal of Strain Analysis, vol. 23, pp. 121-127.

Shukla, A. and Zhu, C. Y. (1988b) "Influence of the microstructure of granular media on wave propagation and dynamic load transfer", Journal of Wave-Material Interaction, vol. 3.

Shukla, A. and Sadd, M. H. (1990) "Wave propagation and dynamic load transfer due to explosive loading in heterogenous granular media with microstructure", AFOSR First Annual Report.

Shukla, A., Sadd, M. H., Xu, Y. and Tai, Q. M. (1993) "Influence of loading pulse duration on dynamic load transfer in a simulated granular medium", Journal of Mechanics and Physics of Solids, vol. 41, no. 11, pp. 1795-1808

Stoll, R. D. (1979) "Experimental studies of attenuation in sediments" Journal of Acoustical Society of America, vol. 66, pp. 1152-1160.

Tai, Q. (1993) "Computer simulation of dynamic load transfer and wave propagation in particulate media", $\mathrm{Ph}$. D . dissertation, University of Rhode Island.

Takahashi, T. and Sato, Y. (1949) "On the theory of elastic waves in granular substance", Bulletin Earthquake Research Institute, vol. 27.

Thornton, C. and Barnes, D. J. (1986) "Computer simulated deformation of compact granular assemblies", Acta Mechanica, vol. 64, pp. 45-61.

Ting, J. M., Corkum, B. T., Kauffman, C. R. and Greco, C. (1989) "Discrete numerical model for soil mechanics", Journal of Geotechnical Engineering, vol. 115 , no. 3, pp. 379-399.

Visscher, W. M. and Bolsterli, M. (1972) "Random packing of equal and unequal spheres in two and three dimensions", Nature, vol. 219, pp. 504.

Walton, O. R., Maddix, D. M., Butkovich, T. R. and Heuze, F. E. (1991) "Redirection of dynamic compressive waves in materials with nearly orthogonal and random joint sets", Proceedings of ASME Applied Mechanics Conference on Recent Advances in Mechanics of Structured Continua, Columbus, $\mathrm{OH}$. 
Wyllie, M. R. J., Gregory, A. R. and Gardner, G. H. F. (1958) "An experimental investigation of factors affecting elastic wave velocities in porous media", Geophysics, vol. 23, pp. 459-493.

Xu, Y. (1991) "Study of Wave Propagation and Dynamic Load Transfer in Heterogenous Granular Media", Ph. D. dissertation, University of Rhode Island.

Zhu, C. Y., Shukla, A. and Sadd, M. H. (1991) "Prediction of dynamic contact loads in granular assemblies", Journal of Applied Mechanics, vol. 58, pp. 341-346. 